\title{
POWER BOAT OPERATORS' VISUAL BEHAVIOR PATTERNS
}

\section{SUSANNE M. GATCHELL, PhD}

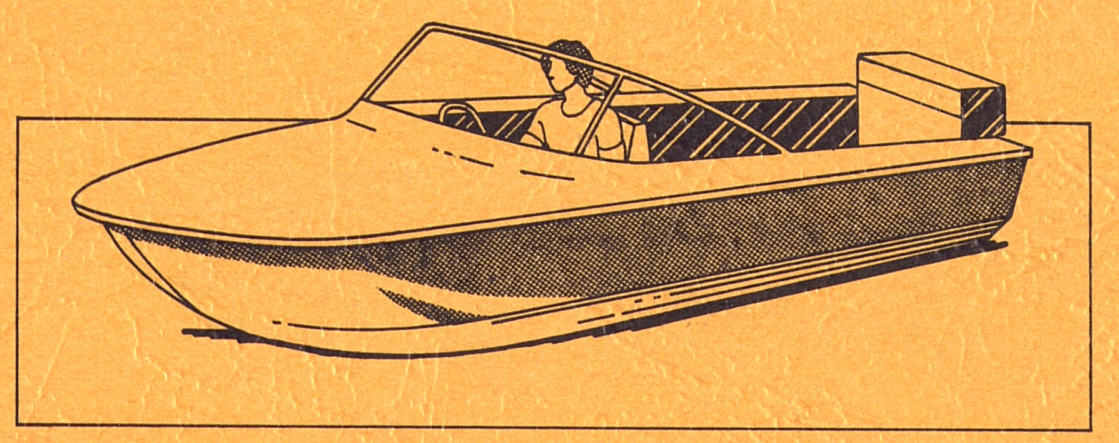

THE UNIVERSITY OF MICHIGAN, 1977 ENGINEERING HUMAN PERFORMANCE AND SAFETY LABORATORY 


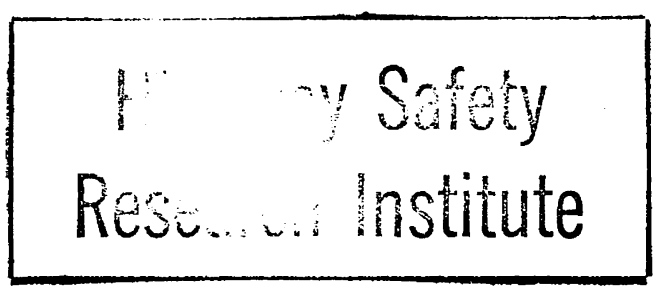




\section{ABSTRACT}

POWER BOAT OPERATORS'

VISUAL BEHAVIOR PATTERNS

by

Susanne Marie Gatche11

Chairman: James M. Miller

The role of vision has been extensively studied in the control of automobiles and aircraft, but little is known about the visual characteristics displayed by recreational power boat operators. Thus, a research effort was structured to: (1) develop a research methodology for collecting boaters' eye fixation data and demonstrate its feasibility; (2) evaluate factors which affect boaters' visual behavior as measured by their eye fixation patterns; and, (3) compare these eye fixation results from boating with similar automobile driver studies where eye fixation data were collected.

To accomplish these objectives, corneal reflection eye fixations were video taped while three experienced boaters performed the following operations under low traffic density situations: three navigation tasks (compass, visual reference point, center in channe1); at three velocities $(29,42,56 \mathrm{kmh})$; and in two boating environments (1imited access, open water). 
A statistical evaluation justifying the adequacy of the subject sample size is presented; and this justification in itself is a contribution which can be generalized for other applications.

Results demonstrated that boaters" eye fixations can be recorded in various conditions with acceptable accuracy; but careful procedures are necessary.

Boaters' fixation durations were not normally distributed and were, thus, analyzed after performing log normal transforms. This finding of non-normality may have general implications to all past and future eye fixation research, since it may not have been given due consideration previously.

Analyses of the data indicate that boaters scanned a significantly larger area to the right front of the vessel during a limited access water condition than during an open water condition: More fixations to the right may be related to the cockpit station being traditionally on this starboard side of the test boat.

During a center in channel task, durations increased with increased velocities. Decreases in durations with increasing velocity levels were exhibited during the compass and visual reference point tasks. Possible explanations for this velocity-navigation task interaction are suggested.

When comparing the visual patterns of boaters with automobile drivers, differences were noted in both the horizontal and vertical fixation coordinates. While centering in a channel, boaters' mean 
horizontal locations were similar to automobile drivers' ( $5^{\circ}$ to the right of center) although their standard deviations were considerably greater $\left(22^{\circ}\right.$ for boaters and $3^{\circ}$ for automobile drivers). Mean vertical locations indicated that boaters scanned below the horizon $\left(-2^{\circ}\right)$, while automobile drivers scanned above the horizon $\left(2^{\circ}\right)$. This may be related to boaters being primarily interested in collision avoidance while automobile drivers are primarily concerned with tracking and lateral placement. 
POWER BOAT OPERATORS'

VISUAL BEHAVIOR PATTERNS

by
Susanne Marie Gatchell

\author{
Susanne Marie Gatchel1
}

A dissertation submitted in partial fulfillment of the requirements for the degree of Doctor of Philosophy (Industrial and Operations Engineering)

in The University of Michigan

1977

Doctoral Committee:

Associate Professor James M. Miller, Chairman Professor Don B. Chaffin

Assistant Professor Gary D. Herrin

Assistant Professor Gary D. Langolf

Professor Richard G. Snyder 



\section{ACKNOWLEDGEMENTS}

This author wishes to express her gratitude to her doctoral committee, especially the Chairman, Dr. James M. Miller, and the other members, Drs. Don B. Chaffin, Gary D. Herrin and Gary D. Langolf of the Department of Industrial and Operations Engineering and Dr. Richard G. Snyder of the Biomedical Department of the Highway Safety Research Institute and the Department of Anthropology

Special thanks are also extended to the following individuals: Mr. James A. Foulke for his development of the visual activity mionitoring system and continued technical assistance in implementing the data collection; Mr. Allan B. Hegg, President of Century Boat Company, for donating a test vesse1 to the University in order that this research may be accomplished; Mr. Daniel R. Dykstra for his assistance in the data collection and data reduction phases; Mr. Robert L. Gebbie for his assistance with the experiments; Ms. Barbara Reed-Polatty and Edith J. Baise for their assistance in typing the numerous drafts; to Ms. Susan S. Moffatt and Danie11i Viecelli for their illustrations; and to the Medical School Human Use Committee for their approval for conducting this research.

This research was partially supported by Contract No. DOT-CG-61098-A from the United States Coast Guard, Century Boat Company, and by Grant No. 2-T01-0H-00161-04 from the National Institute for Occupational Safety and Health. The author also wishes to express her gratitude to General Motors Design Staff for sponsoring a significant portion of her graduate education. 
TABLE OF CONTENTS

ACKNOWLEDGEMENTS

LIST OF TABLES

LIST OF FIGURES

ii

vi

CHAPTER I: BACKGROUND AND LITERATURE REVIEW 1

Introduction

Literature Review

1

Boating Research

Automotive Research

Automotive Eye Fixation Research 15

CHAPTER II: RESEARCH APPARATUS $\quad 20$

$\begin{array}{ll}\text { Test Boat } & 20\end{array}$

Visual Activity Monitoring System 25

Eye Marker Calibration 31

CHAPTER III: RESEARCH METHODOLOGY

Independent Research Variables 33

Navigation Tasks $\quad 34$

Velocity ... $\quad 36$

Boating Environment $\quad 37$

$\begin{array}{ll}\text { Subjects } & 37\end{array}$

Experimental Design $\quad 38$

Uncontrolled Measured Variables $\quad 42$

Test Location $\quad 43$

Test Procedures $\quad 51$

Pre-Test Subject Preparation Phase 51

Familiarization Phase $\quad 54$

Coast Guard Data Collection Phase $\quad 55$

Experimental Data Collection Phase 56

CHAPTER IV: RESULTS $\quad 58$

Data Reduction $\quad 58$

Fixation Definition $\quad 61$

Fixation Location Determination 63

Fixations Eliminated from Data Sets $\quad 65$

Analysis of Results 68

Spatial Patterns - Horizontal and Vertical

Navigation Task Effects . 70

Boating Environment Effects $\quad 74$

Subject Effects $\quad 74$

Subject-Boating Environment Effects 74

Components of Variance for Horizontal Location 78

Spatial Patterns - Eye Spot Travel Distance _. 80

Subject. Effects $\quad 80$

Boating Environment Effects $\quad 81$

Temperal Chatacteristics - Fixation Durations $\quad 82$

Fixation Targets $\quad 84$

Analyses with Navigation Targets Excluded 84

Analyses with Navigation. Targets Included $\quad 88$ 
Table of Contents (cont.)

CHAPTER V: COMPARISON TO AUTOMOTIVE EYE FIXATION RESEARCH 91

Spatial Chatacteristics 93

Temporal Characteristics $\quad 94$

Summary of Comparison Characteristics 98

Spatial $\quad 98$

Temporal $\quad 99$

CHAPTER VI: SUMMARY AND RECOMMENDATIONS 100

Objective \#1: Collection of Boater Eye Fixation $\begin{aligned} & \text { Data } \\ & 100\end{aligned}$

Objective 非: Some Factors Affecting Boaters'

Visual Behavior 101

Boating Environment Navigation Task Effects · 101

Fixation Locations 101

Fixation Durations 102

Subject Effects . 103

Objective \#3: Comparison of Boater Vs. Automotive

Visual Behavior Patterns 104

Future Research Recommendations

Recommendations Related to Literature Review 104

Recommendations Related to Resultant Boating

Data 105

$\begin{array}{ll}\text { BIBLIOGRAPHY } & 107\end{array}$

APPENDIX 


\section{LIST OF TABLES}

Table

Page

1.1 Results from three Rockwe11 Eye Movement Studies

3.1 Independent Variables

Analysis to Determine Number of Subjects

3.6 Environmental Conditions Recorded for Each Test Run

4.1 Criteria for Data Selected for Reduction

59

4.2

Dependent Measures Recorded by Data Reducer

4.5 Significant Results from the Analysis of Variance for Horizontal Location of Eye Fixations

4.6 Significant Results from the Analysis of Variance for Eye Spot Travel Distances

5.1 Comparison of Boat and Automobile Operator's Eye Fixation Data 


\section{LIST OF FIGURES}

Figure

Page

1.1: Operators' primary search directions to detect potential collision vehicles

1.2: Photograph illustrating vehicle produced glare in the boating environment.

2.1: Original test boat $\quad$ - 21

2.2: Modified test boat 22

2.3: Original and modified instrument panel 24

2.4: Layout of experimental boat 26

2.5: " Illustration of visual activity monitoring helmet - 27

2.6: Illustration of visual activity monitoring system 28

2.7: Illustration of VAM eye marker system TV picture. 30

3.1: Test site location 45

3.2: Boating test course $\quad 47$

3.3: Limited access boating test course 48

3.4: Open water boating test course 49

4.1: Spatial reference coordinates 60

4.2: Histograms of horizontal location including and excluding compass fixations

4.3: Representative distribution of horizontal fixation locations

4.4: Navigation task effects on spatial fixation coordinates 73

4.5: Boating environment effects. on spatial fixation coordinates

4.6: Subject effects on spatial fixation coordinates $\quad 7.6$

4.7: Subject-boating environment effects on spatial coordinates

4.8: Components of variance for horizontal locations 79

4.9: Fixation duration distribution 83

4.10: Histogram of lognormal transform of duration data . 85

4.11: Illustration of fixation durations for velocitynavigation task interaction

4.12: Percent fixation time by target type for each subject

4.13: Percent fixation time by target. type for subjects and navigation tasks.

5.1: Elliptical illustration of automobile drivers' and boaters' spatial scanning patterns

5.2: Velocity effects: boating center in channel task vs. McDowel1 (1975) automobile driving 
CHAPTER I

BACKGROUND AND LITERATURE REVIEW

\section{INTRODUCTION}

Extensive research has been performed to investigate behavior patterns of operators in various transportation modes (primarily aircraft and automobile); however, little is known about the behavior patterns of recreational power boat operators. The problems of these boat operators are just beginning to receive attention. The United States Coast Guard has recently developed an interest in the human factors aspects of smallcraft operators, and is supporting research in hopes of determining causal relationships between the behavior of operators and boating collision accidents. It will, however, take many years of extensive and expensive research to gain an understanding of boaters which is even comparable to our limited understanding of automobile or aircraft operator behavior. To assist in this definition of the boater's behavior, it would be advantageous to utilize that research which is applicable from these other transportation modes.

Vision is an important sensory modality for vehicle operators; and determining eye fixation points is a method which has proven particularly successful in quantifying the visual behavior of automobile drivers. Thus, in order to explore similar visual behaviors in boaters, the research reported herein was undertaken with the following research objectives: 
1. to develop a research methodology and establish the feastbility of collecting boaters' eye fixation data;

2. to evaluate several factors which affect boaters' eye flxations; and

3. to compare these eye fixation results with similar automobize drivers studies where eye fixation data were collected.

The literature review section develops reasons for these objectives and discusses why the comparisons are limited to automobile drivers.

\section{LITERATURE REVIEW}

\section{Boating Research}

Boating human factors research was initiated by Miller (1973) who applied the knowledge and techniques gained from experiments in other environments to the boating arena. His research included extensive literature reviews in the area of stressors (e.g., heat, vibration, noise, etc.), perception, decision making, anthropometrics, and cockpit design practices. He also performed an in-depth statistical re-analysis of the 1972 Coast Guard Boating Accident Report (Miller, 1973). Finally, future research needs were proposed which included recommendations to study visibility related problems which might lead to collisions.

The following results were among those reported by Miller in his analysis of the 1972 Coast Guard Boating Accident Report data: 
1. Of the 4308 vessels having damage, injuries or fatalities the following was reported:

a. $78 \%$ of the operators had 100 hours or more boating experience.

b. In $79 \%$ of the cases, the weather and visibility were good.

c. In $56 \%$ of the cases, the water was calm, while only $24 \%$ of the cases reported the water condition as choppy.

d. In $63 \%$ of the cases, the wind was reported as none to 1ight.

2. Of the 120 "Other Deaths," $45 \%$ of the vessels had a col1ision with another boat or an object.

3. Of the 3127 vessels damaged, $50 \%$ were cruising at the time of the accident, and $49 \%$ had a collision with another vesse1.

It was not unskilled beginning boaters who lost control of their vessels in rough water and who caused the majority of accidents. Rather, it was experienced operators, cruising in other than rough water, who collided with another object which they either 1) did not see in time to avold, 2) did not recognize as being on a collision course with then, or 3 ) did not know how to avoid, with their particular skill, knowledge, or experience level.

As a result of this initial focus on the collision problem, the next follow-on study as reported by MacNeil1, et al., (1975) 
attempted to further identify causal factors in collision accidents in accordance to some of the recommendations made in the Miller 1973 reference. In analyzing 55 collisions reported to the Coast Guard in 1974, MacNeill (1975, p 55) stated that "inattention was... the primary cause for $22 \%$ of the collisions." This inattention can be interpreted as operators failing to observe, process or act on the visual information which should have been used to avold the collision.

As a result of ten in-depth investigations involving 15 boats, MacNeill in the next report $(1976 \mathrm{~b}$, p. 9-10), followed this "inattention" suggestion and found that:

"Visibility oriented problems were identified as
causing the collision in $94 \%$ of the cases; broken down as follows:

- he didn't see boat/object in time to avoid it but tried to
didn't try

- he didn't see boat/object at all because: he wasn't looking

his vision was obscured $20 \%$

it $\operatorname{wasn}^{\prime} t$.visible $13 \%$

In the latest series of studies under Coast Guard sponsorship, MacNeil1, et al., (1976a) also discussed the series of three tests which used a Visual Alertness Stress Test (the VAST system). This VAST system consisted of a $5.2 \mathrm{~m}$ (17 ft.) boat with a center helm 
position surrounded by a semicircular light display. Subjects were instructed to steer a particular course and respond to the stimulus lights by depressing a button on the throttle. A primary function of this system was to test fatigue effects on peripheral vision response times. (In order to induce fatigue, subjects spent three hours performing specific activities, such as playing baseball, riding in a boat, etc.) The original idea for the VAST experiment was conceived by J. Miller, G. Herrin and S. Gatchell while acting as consultants to Wyle Laboratories. The engineers at Wyle then refined the concept and implemented it in the present form of the VAST boat. The reported results of the VAST-1 test indicated that in the fatigued states the six subjects had significantly more missed signals and slower response times. For example, boaters' reaction times doubled from $2100 \mathrm{msec}$ in the rested state to $4000 \mathrm{msec}$ in the fatigued state. The second experiment (VAST-2) studied the primary and synergistic effects of fatigue and alcohol. Results indicated that fatigue still had a significant effect although not as large as in VAST-1 (RT's increased from 1800 to $2000 \mathrm{msec}$ in the fatigued state). It was also found that there was a significant effect due to alcohol and an interaction effect between fatigue and alcohol.

VAST-3 was an ambitious undertaking which attempted to study alcohol, fatigue, noise, shock/vibration, glare and their interactions in a three subject experiment. These factors were thought to be major among the important potential stressors in boating. The results yielded no single factor which consistently degraded error rate or 
response times. Alcohol was statistically significant as a main effect on response time performance, but it "improved" response time performance at the middle $.05 \% \cdot 1$ evel.

These studies, by MacNei11, et al:, (1976a) using the VAST system, imparted a simulation type environment on a boating task, the fidelity of which might be questloned. Moreover, the subjects in the VAST-1 task were al1 Coast Guard personnel and in the VAST-2 and VAST-3 were Wyle personnel. Selecting subjects in this nature may have resulted in a biased subject pool which is not representative of the average smallcraft boater. In performing further boating research studies, it would be advantageous to get a more representative subject population.

While performing the VAST task, the subject"s primary task was to maintain compass headings. Their secondary task was to perform the VAST task. However, analytical judgments were never made as to the degree or percentage of time that subjects spent on the primary vs. secondary tasks. Given there was enough latitude maintaining compass headings and that the boating situation was non-stressful, then it would be feasible to assume that subjects spent a larger percentage of their time monttoring the VAST apparatus than on their primary task of maintaining compass heading.

Traffic density would seem to be an important factor in operators' visual behavior related to collision avoidance but MacNei11 never mentions the traffic density characteristics in the immediate test site during any of these VAST s.tudies. 
Another methodology used to determine boaters' behavior is a simple photographic survey of boaters. Sowa and Fraser (1974) observed 156 smallcraft boaters, and found that approximately $13 \%$ were sitting on the top of the seat back; while MacNeill's (1976a, p. 113) survey of 270 boaters "showed that $1 / 3$ of the operators were standing, kneeling, etc. in order to get their eye point high enough to see adequately." Operators in this type of position, although achieving better external visibility, reduce their ability to reach and operate their controls.

Other methods besides those mentioned above are available for gaining more quantitative information of boaters' visual responses but have not as yet been attempted. In particular, many researchers have utilized an eye fixation apparatus to study automobile drivers' visual behavior. This technique seems particularly suited to gain additional information about boat operators. Thus, the first research objective for this research has been chosen as follows:

OBJECTIVE 1: DEVELOP A RESEARCH METHODOLOGY AND ESTABLISH THE FEASIBILITY OF COLLECTING BOATERS' EYE FIXATION DATA.

Because of the research precedence established in the automobile arena, the following section examines, first, some relevant automobile driver research, and then discusses some specific automotive eye fixation studies which may provide insight into what might be expected from boat operators. 


\section{Automotive Research}

Due to the cockpit similarities, and since adult boat operators are also experienced automobile drivers, one might expect that a large portion of boat operators" behavior may result from a "transfer of training" from automobile driving. However, boaters should compensate for the differences between the two environments when driving a boat.

Differences between these two types of operations become apparent when one considers the primary tasks. McDowell (1975, p. 38). summarized the task of automobile driving as follows:

"1. Driving is primarily a preview control task where the driver previews the roadway ahead and attempts to minimize the deviation between the vehicle's actual state and the desired state over some time interval.

2. The task is primarily two dimensional with the driver controlling the vehicle's lateral position and velocity.

3. The driver is a discrete data sampling controller, as opposed to a continuous process monitor, with vehicle. dynamics and roadway geometry playing an important role in determining the sampling strategy."

Many of the automobile drivers' tasks are necessitated by the fact that they have a limited, confined path in which to maneuver their vehicles. Boat operators have more flexibility in lateral positioning and velocity maintenance, thus, navigation may not be their primary task. Instead, collision avoidance may be the primary task for boaters. This is necessitated by the fact that many potential non-vehicular collision objects (e.g., logs or debris) are difficult to see in the water. Automobile drivers are concerned with similar 
collision avoidance, but given that they stay in their limited tracking area, there is a lower probability that a potential non-vehicular object will be in their path. In discussing automobile drivers' detection of obstacles in their roadway, McDowell (1975) assumes that detection is not difficult; instead, the drivers are faced with the greater task of deciding the necessary control action required by the situation. This detection process may be extremely difficult for boaters because many potential non-vehicular obstacles may be partially or totally submerged in the water. Even those obstacles which are above the water may be difficult to detect due to glare or low contrast ratios with the surrounding water.

In their searching and scanning behavior, automobile drivers are aided by mirrors which have been studied by many researchers (Pettit, 1966; Marcus, 1968; Mansour, 1971; and Mourant and Donahue, 1974). The amount of time that automobile drivers spend fixating to the mirror depends on their immediate driving task. Mourant and Donahue (1974) studied two such mirror systems, one with a $25 \% 1$ arger field of view than the other, and found no differences in fixation durations and frequencies to either mirror. This suggests that automobile drivers do not gaịn additional information from larger mirror systems, but rather within each task they need a fixed amount of time to acquire rear visual information. Unfortunately for boaters, this type of mirror system has limited availability and usefulness; and obtaining information from a rear vịsual system on a boat may be hindered by vibration transmitted to the mirrors. 
Automobile drivers need to search primarily for vehicles in limited areas (forward, directly to the rear and $90^{\circ}$ to the sides). In contrast, boaters should search for potential collision vehicles anywhere within the $360^{\circ}$ area surrounding their vessel, as illustrated in Figure 1.1. Thus, the dispersion of the visual search pattern should be greater for boaters than automobile drivers because 1). potential collision obstacles can impinge from a greater number of locations than in the automobile driving situation and 2) they do not have a mirror system.
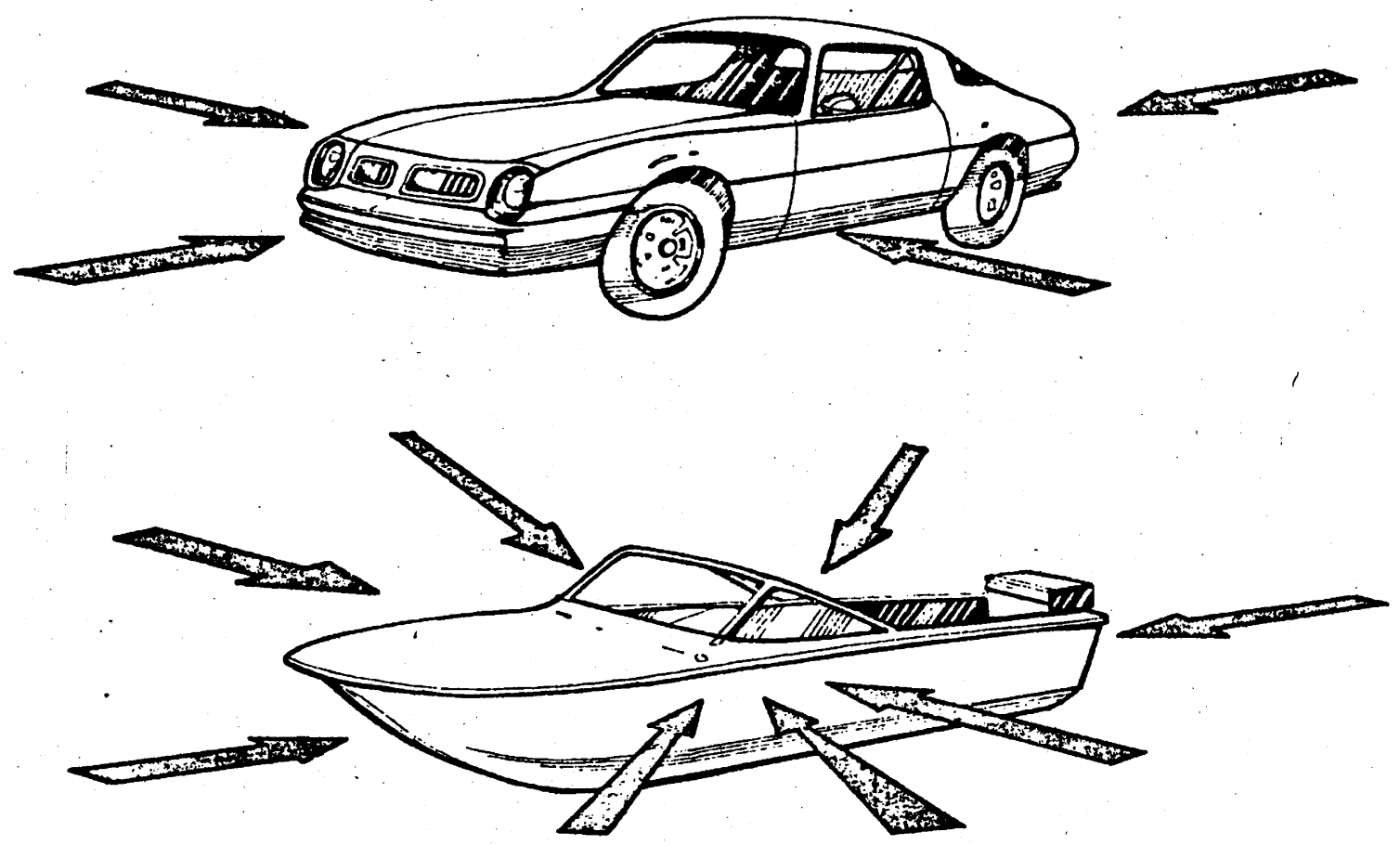

Figure 1.1: Operators': primary search directions to detect potential collision vehicles 
In determining where one would expect operators to scan, it is also important to know the types and probable locations of task related information. This was accomplished for automobile drivers by Ford Motor Company (1972). A 2400 mile photographic survey was undertaken to determine those areas around an automobile where drivers were most likely to view such objects as other vehicles, traffic signs and signals or pedestrians. It is undesirable to place vehicular structures in locations which would obstruct driver vision to such objects. A method for accessing the obstructed and nonobstructed visual areas for automobile drivers was developed by Barnoski, et al., (1970). His method allows one to make objective visibility comparisons between vehicles by assigning a number between 0 and 100 to the particular vehicle being evaluated. Boats have similar problems to automobiles in that they contain pillars and other structures which can interfere with driver visibility. Dissimilarity arises from visual obstructions caused by the changing planing angles of the boat. A computer graphic method for assessing this type of visual problem was developed by Miller (1973).

In addition to potential structural interference, there are also possible visual problems related to the foveal and peripheral capabiltties of individual drivers. Salvatore (1968) used subjects seated as passengers in an automobile with their heads in a chin rest. He found that individuals could estimate a vehicle's velocity better 
with peripheral cues than foveal. Newsome (1967) determined that the further in the periphery an object was, the further away an observer. jưdged its distance; an object at a peripheral angle of $180^{\circ}$ was judged by observers to be 100 feet away when the correct distance was only 65 feet. Glasses can also restrict peripheral vision capabilities (Smith and Weale, 1966 and Bewley, 1969). Burg (1968a) reported that age will cause a decrease in the lateral visual field. From these above findings, one can infer, for example, that boaters might have a tendency to underestimate the hazards associated with a boat seen in the periphery. Moreover, velocity estimates may be more difficult in open water, where the relevant peripheral cues similar to a traffic roadway are not prevalent.

Detection problems may be further apparent when one considers night boating. Night myopia has been detected in young automobile drivers; and, positive after images can result as an automobile driver looks directly at oncoming headlights (Fry, 1968). Dark adaptation is also a problem in automobile driving. This is definitely age related, where Domey and McFarland (1961) have recorded that it takes a teenager 10 minutes to become dark adapted and a 60 year old 28 minutes.

One might expect these adaptation problems to be prevalent during night boating, which in themselves makes the detection of collision obstacles difficult. However, these problems are compounded by irrelevant light sources. Even if boaters detect an approaching 
vehicle, they can easily lose sight of it if its background has several light sources, such as those emitted from shore. Additional boating problems were reported by Stiehl (1975). In a survey of 150 people involved in nighttime boating accidents, he noted that the glare produced by the $360^{\circ}$ stern light (manditory on boats) was a common problem in these collisions. Many boaters travel at night without their stern light on in order that they might be able to detect other boats. Problems arise when two boats in the same vicinfty are traveling without using these stern lights. Stiehl indicated that $42 \%$ of the accident involved persons surveyed sald that the other boat's 1ight were off. Judging whether another boat's lights were on can be difficult for operators depending on 1) the number of Irrelevant lights in the background and 2) whether they were looking in the direction of the approaching vessel. of course, it is easier to get these operators to admit that someone else's lights were not on.

Another boating problem is related to glare. Glare interferes with visual detection due to the scattering of light on the retina. Burg (1968b) analyzed drivers' visual performance and its relationship to accidents. He used a measure of glare recovery and found that it was a predictor of accident rate. This, however, did not predict as well as his dynamic visual acuity measure. MacNei11, et al., (1976a) felt that the glare factor was important enough to include it in the VAST-3 study. In controlling for glare in this VAST-3 study, subjects either did or did not wear sunglasses. MacNeill 
never mentioned the visual properties of the sunglasses; thus, replications of this experiment would be difficult.

Automobile manufacturers found that a large amount of glare comes from the vehicle structure itself. The Motor Velicle Safety Standard No. 107 (1968) addresses this problem with respect to such things as the finish on metal objects which could reflect in drivers' eyes (i.e., windshield wiper arms). Similar vehicle glare problems are particularly relevant in the boating arena. Boat manufacturers do not utilize much glare reducing material and still insist on many chrome objects around the boat, particularly in the instrument panel area. Figure 1.2 is an illustration of the effect of glare on the windshield.

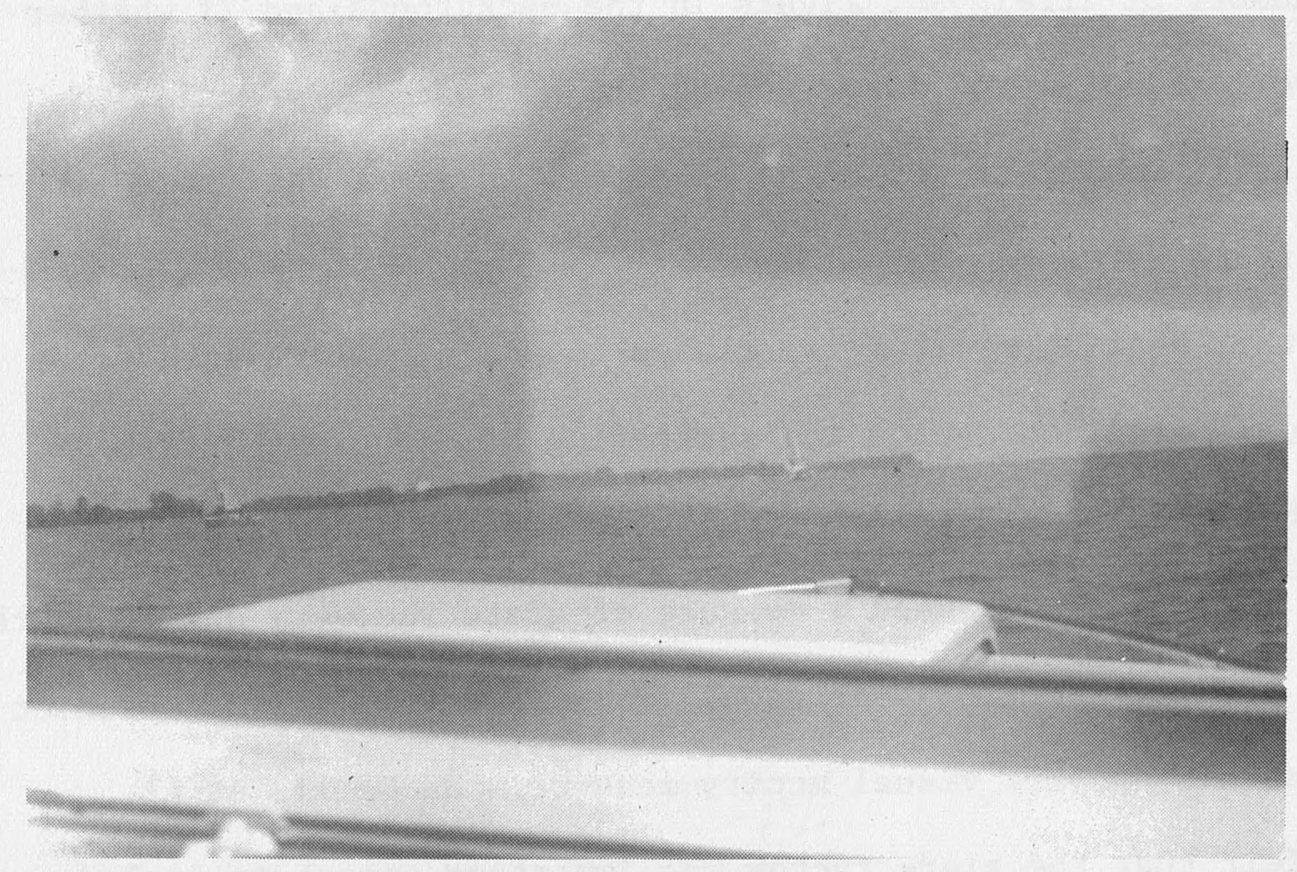

Figure 1.2: Photograph illustrating vehicle produced glare in the boating environment 
The previous section discussed some of the automotive research which has relevance to the boating arena. The following section contains examples of various types of automotive eye fixation research.

\section{Automotive Eye Fixation Research}

Numerous automotive eye fixation type studies have been performed by Rockwe11 and others at Ohio State University (e.g., Rockwe11, Overby and Mourant, 1968; Rockwe11, Ernst and Ru1on, 1970; and Ze11, Rockwel1 and Mourant, 1969). Using a corneal reflection eye marker system, Rockwe11 and others have been able to determine areas where drivers fixate to during different types of tasks. Some of their results are summarized in Table 1.1. From this table, the most noticable inference is that the drivers' time is spent primarily looking at objects in a straight ahead viewing area $\left(-3^{\circ}\right.$ to $5^{\circ}$ azimuth and $-2^{\circ}$ to $2^{\circ}$ elevation). This is true whether the drivers are on an open freeway, changing lanes on a freeway, following a car or driving in a neighborhood area. The familiarity of the route does not greatly affect the viewing area, although there does seem to be a downward trend of fixation location with repeated familiarity (Mourant, et al., 1969). It is also obvious from the fixation duration results in Table 1.1 that automobile drivers spent most of their time looking straight ahead. Less than $10 \%$ of their time was spent looking at road signs of lane markers. 
Table 1.1

Results from three Rockwell Eye Movement Studies

\begin{tabular}{|c|c|c|c|c|}
\hline Study & $\begin{array}{l}\text { Swrwher of } \\
\text { Subject: }\end{array}$ & Task & $\begin{array}{l}\text { Mean or Medtan } \\
\text { of Vleulng Angle }\end{array}$ & $\begin{array}{l}\text { Fixation } \\
\text { Time Results }\end{array}$ \\
\hline \multirow{5}{*}{$\begin{array}{l}\text { Whalen, Rockwell and } \\
\text { Mourant (1968) "A } \\
\text { P1lot Study of Drivers" } \\
\text { Eye Moveneats" }\end{array}$} & 3 & $\begin{array}{l}\text { Highway Driving } \\
\text { Open Road } \\
\text { (50 aph.) }\end{array}$ & $\begin{array}{l}5^{\circ} \text { azlmuth, } \\
0^{\circ} \text { elevation }\end{array}$ & \multirow{5}{*}{$\begin{array}{l}\text { Medtinn of Fixation } \\
\text { Duration for all } \\
\text { tasks was } 1 / 4-1 / 2 \\
\text { second. }\end{array}$} \\
\hline & & $\begin{array}{l}\text { Open Road } \\
(70 \text { aph. })\end{array}$ & $\begin{array}{l}5^{\circ} \text { azinuth, } \\
2^{\circ} \text { elevation }\end{array}$ & \\
\hline & & $\begin{array}{l}\text { Car Following } \\
\text { ufth Short } \\
\text { Headway }\end{array}$ & $\begin{array}{l}6^{\circ} \text { aztinuth, } \\
0^{\circ} \text { elevation }\end{array}$ & \\
\hline & & $\begin{array}{l}\text { Overtak1ng a } \\
\text { Lead Ing Vehtcle }\end{array}$ & $\begin{array}{l}5^{\circ} \text { azlinuth, } \\
0^{\circ} \text { elevation }\end{array}$ & \\
\hline & & $\begin{array}{l}\text { Freeway Traffic } \\
\text { Driving }\end{array}$ & $\begin{array}{c}5 \cdot \text { ezimuth, } \\
-1^{-} \text {elevation }\end{array}$ & \\
\hline \multirow{10}{*}{$\begin{array}{l}\text { Mourant, Rockwell } \\
\text { and Rockoff (1969) } \\
\text { "Drivers' Eye Move- } \\
\text { meat and v1sual } \\
\text { Workload" }\end{array}$} & 8 & $\begin{array}{l}\text { Open Freeway } \\
\text { Driving }\end{array}$ & & $\begin{array}{l}\text { S0z of Viewing Time } \\
\text { Looking Ahead }\end{array}$ \\
\hline & & Trial $\| 1$ & $\begin{array}{l}5^{\circ} \text { azimuth, } \\
2-1 / 2^{\circ} \text { elevation }\end{array}$ & $\begin{array}{l}\text { 8: of VLeulng TIme } \\
\text { Looking at Bridgea }\end{array}$ \\
\hline & & Trial /2 & 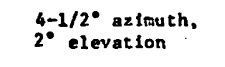 & $\begin{array}{l}\text { 6\% of Viewing Time } \\
\text { Looking at Road Signs }\end{array}$ \\
\hline & & Trial 3 & $\begin{array}{l}4-1 / 2^{\circ} \text { azimuth, } \\
1^{\circ} \text { elevation }\end{array}$ & $\begin{array}{l}\text { 3\% of Vlewing Time } \\
\text { Looking at Vehicles }\end{array}$ \\
\hline & & & & $\begin{array}{l}2 z \text { of Viewing TIme } \\
\text { Look Ing at Road and } \\
\text { Lane Markers }\end{array}$ \\
\hline & & $\begin{array}{l}\text { Car Following } \\
\text { (Freeway Difiving) } \\
\text { Trial il } 1\end{array}$ & $\begin{array}{l}4^{\circ} \text { azimuth, } \\
1^{\circ} \text { elevation }\end{array}$ & $\begin{array}{l}\text { 40\% of Vleulng Time } \\
\text { Looking at Lead Car } \\
\text { and Other Vehicles }\end{array}$ \\
\hline & & Trial 12 & $\begin{array}{l}4-1 / 2^{\circ} \text { aztimuth, } \\
0^{\circ} \text { elevation }\end{array}$ & $\begin{array}{l}\text { 30\% of Vicwing Time } \\
\text { Look ing Ahend }\end{array}$ \\
\hline & & Trial & $\begin{array}{l}4^{\circ} \text { az Imuth, } \\
0^{*} \text { elevat ion }\end{array}$ & $\begin{array}{l}\text { SR of Vlewlng Time } \\
\text { Lnoking at Bridges }\end{array}$ \\
\hline & & & & $\begin{array}{l}4 \% \text { of viewing Iime } \\
\text { Looking at Road SIgns }\end{array}$ \\
\hline & . & & & $\begin{array}{l}32 \text { of Viewink Time } \\
\text { Look ing at Road and } \\
\text { Lane Markers }\end{array}$ \\
\hline \multirow[t]{8}{*}{$\begin{array}{l}\text { Mourant and Rockuell } \\
\text { (1972) "Strategles of } \\
\text { V1sual Search by } \\
\text { Novice \& Experienced } \\
\text { Drivers" }\end{array}$} & \multirow[t]{8}{*}{$\begin{array}{l}\text { Exper- } \\
\text { lenced } \\
\text { Driver: }\end{array}$} & $\begin{array}{l}\text { Ne1Rhborhood } \\
\text { Task } \\
\text { Approach to } \\
\text { Stop Sign }\end{array}$ & $\begin{array}{l}0^{\circ} \text { azinuth, } \\
0^{\circ} \text { elevation }\end{array}$ & . \\
\hline & & $\begin{array}{l}\text { Approach to } \\
\text { Traffic Llght }\end{array}$ & $\begin{array}{l}-3^{\circ} \text { azimuth, } \\
0^{\circ} \text { elevation }\end{array}$ & \\
\hline & & $\begin{array}{l}\text { Approach to } \\
\text { Left Turn }\end{array}$ & $\begin{array}{l}2^{\bullet} \text { azlnuth, } \\
-1^{\circ} \text { elevation }\end{array}$ & \\
\hline & & $\begin{array}{l}\text { Approach to } \\
\text { Righe Turn }\end{array}$ & $\begin{array}{l}6^{\circ} \text { azimuth, } \\
-1^{-} \text {elevation }\end{array}$ & \\
\hline & & $\begin{array}{l}\text { Freewsy Task } \\
\text { Changing to } \\
\text { Left Lane }\end{array}$ & $\begin{array}{l}-2^{*} \text { Azinuth, } \\
-2^{\circ} \text { el evition }\end{array}$ & $\begin{array}{l}9 \text { suc. mean oilance } \\
\text { durution it linside } \\
\text { reistediew mitror }\end{array}$ \\
\hline & & $\begin{array}{l}\text { Chanzing to } \\
\text { Right Lane }\end{array}$ & $\begin{array}{l}1^{\bullet} \text { azisinth. } \\
1^{-} \text {elevation }\end{array}$ & $\begin{array}{l}\text { 1.0 sec. mean flanice } \\
\text { duration at slde } \\
\text { mirror }\end{array}$ \\
\hline & & $\begin{array}{l}\text { Travel ing in } \\
\text { Left Lane }\end{array}$ & $\begin{array}{c}2^{\circ} \text { azinuth, } \\
-1^{\circ} \text { elevation }\end{array}$ & $\begin{array}{l}\text {. S suc. mean glance } \\
\text { duration at speedn- } \\
\text { meter }\end{array}$ \\
\hline & & $\begin{array}{l}\text { Iraveling In } \\
\text { RIght Lane }\end{array}$ & $\begin{array}{l}3^{\circ} \text { az lnuth. } \\
-1^{\circ} \text { elevation }\end{array}$ & \\
\hline
\end{tabular}


In the Kaluger and Smith study (1970), fatigue caused the eye fixation patterns to be less concentrated (i.e., scanned a larger area), indicating that the fatigued drivers probably had to use foveal vision in the areas typically monitored peripherally.

McDowe11 (1975) reported that fixation durations were longer with increased velocity and suggested that this was related to operators processing information more accurately at higher velocities. Such velocity effects may be particularly pertinent in boating, due to the fact that the operators' have the freedom to select their speed in most types of boating environments.

Bhise (1973) studied automobile drivers as they merged onto freeways via a ramp. He noted that drivers on the entrance ramp made considerably more use of their side view mirror than when they were on the freeway.

Automobile driver's eye fixation patterns have also been studied for other types of roadway geometry. Shinar, et al., (1977) found that drivers approaching a curve alternate their fixations between the road ahead and the right road edge.

Additional studies have been performed to investigate the role of carbon monoxide, marijuana and alcohol on automobile drivers! eye fixations. Rockwell and Weir (1973) found that with elevated carbon monoxide levels drivers increased their percent of fixations in the looking ahead area. This was suggested as being related to a type of perceptual narrowing which developed as the level of carbon monoxide 
increased. Moskowitz, et al., (1976) had subjects drive a simulator while recording their eye fixation patterns; they reported an increased dwe11 (duration) time with alcohol and a decreased fixation frequency. These authors suggest that this alcohol effect is related to a decreased information processing rate. In the same simulator but with different subjects, marijuana did not produce the effects that Moskowitz had reported with alcohol. In fact, none of the eye fixation dependent measures exhibited any significant effects due to marijuana (Moskowitz, et al., 1976).

The above automotive driver eye fixation studies reviewed the results from a variety of independent variables. In order to gain a comparable understanding of boaters, research objective \#2 was undertaken.

OBJECTIVE 非: TO EVALUATE SEVERAL FACTORS WHICH AFFECT BOATERS! EYE FIXATIONS.

Discussing the results from objective 非 with respect to some of the above automotive studies occurs as the third research objective. OBJECTIVE 非: TO COMPARE THESE EYE FIXATION RESULTS WITH SIMILAR AUTOMOBILE DRIVERS' STUDIES WHERE EYYE FIXATION DATA WERE COLLECTED.

Regarding other than the automotive arena, airplane pilots have detection problems similar to boaters, in that they must scan their exterior environment for potential collision objects (i.e., other planes, etc.). However, researchers who have studied these pilots have concentrated primarily on their instrument scanning behavior 
and not on their external fixations (Fitts, et al., 1950; Jones, et a1., 1949; and Senders, et al., 1966); thus, the findings from these researchers have limited application to boaters and will not be discussed. The following section describes the changes which were incorporated into the research apparatus in order to make it possible to determine boaters' eye fixations. 


\section{CHAPTER II}

\section{RESEARCH APPARATUS}

This chapter pertains to the portion of Objective 1 1 related to the feasibility of collecting boaters' eye fixation data. For the purpose of recording boaters' eye fixations, two major pieces of equipment were necessary: an eye marker system, and a test boat. Each of these items is discussed below.

\section{TEST BOAT}

A 6 meter (22 ft.) cabin motor boat was donated to the University by Century Boat Company for the purpose of performing operator visibility related research (see Figure 2.1). Although this vessel is larger than the average size boat, it was selected for the following reasons :

1. The delicate nature of the electronic data collection equipment required that it be protected from water, extreme vibrations, and engine electrical interferences. Since this test boat had a more stable ride than smaller boats, vibrations on the equipment were minimal.

2. The hardtop and glassed-in-areas offered more protection to the subject and test equipment; and the hardtop reduced some of the glare on the subject.

3. The vessel was large enough for three experimenters to perform different tasks without distracting the subject from his primary task of driving the boat. 


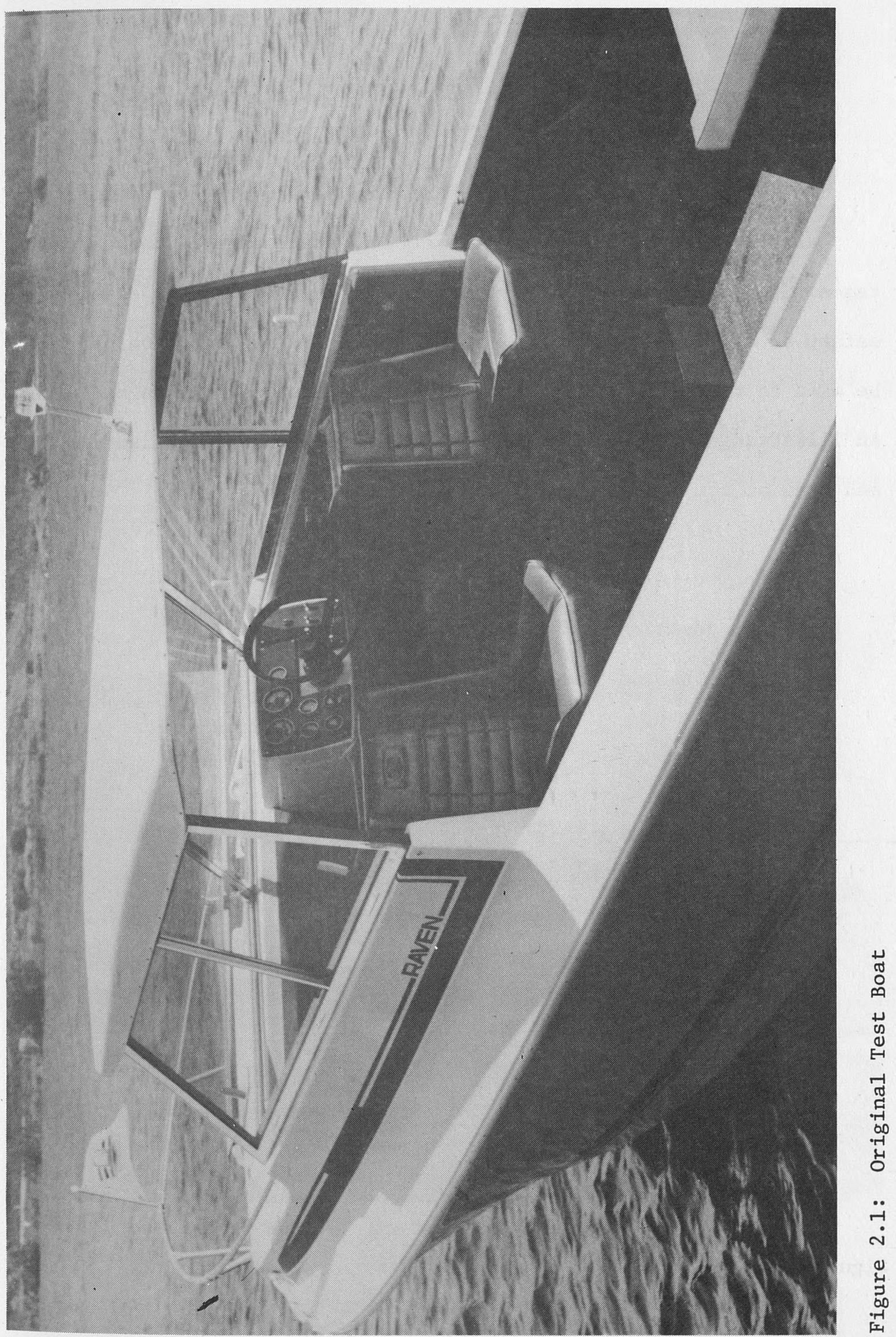


4. The forward located starboard helm position was similar to many popular boats in the 4.9 to 7.9 meter ranges and the research results might, therefore, be fairly representative.

Although the test boat in its original state offered many advantages, it did not fully satisfy the experimenters as to the ease and safety of conducting the study. Thus, extensive modification had to be made to this vessel before any data could successfully be collected. An illustration of the modified test boat is contained in Figure 2.2 and some of the modifications are as follows:

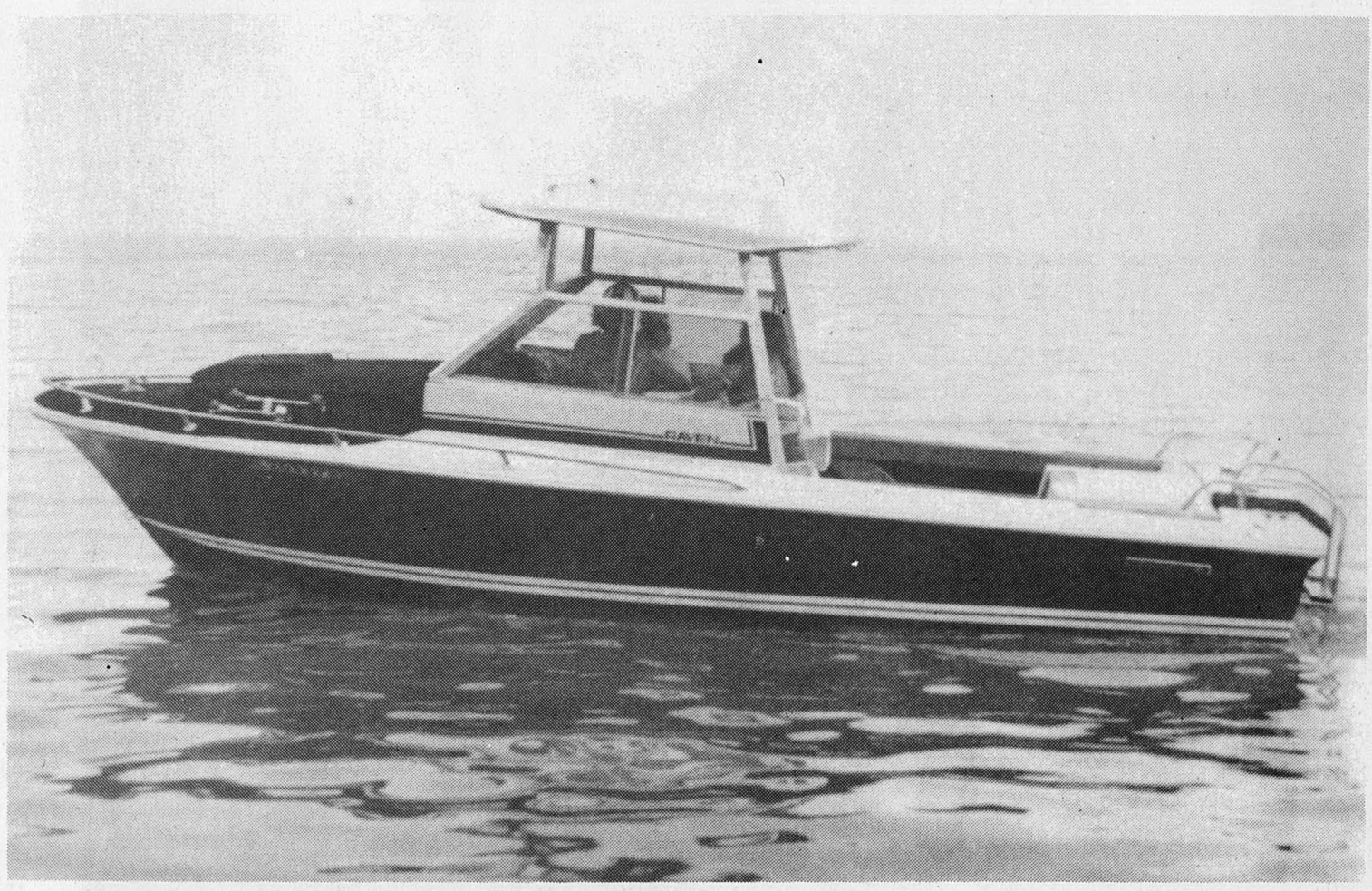

Figure 2.2: Modified test boat 
1. The driver's seat was a pedestal type seat with fore and aft seat adjustment. It was modified to include vertical seat adjustment. Thus, if the subject felt his seated position height was not optimal, he could raise or lower the seat. This was necessary in order to improve the forward visibility of. the driver while trying to scan the water.

2. Gauges and controls on the instrument panel were relocated to improve visibility and ease of operation. The original and the modified instrument panels are illustrated in Figure 2.3 .

3. Glare reducing material was installed on the bow of the boat, the underside of the roof, the instrument panel and several chrome areas. which were glare sources (e.g., the spokes of the steering whee1).

4. The roof of the boat was raised 15". This was necessary in order to provide enough head room such that the driver while wearing the corneal reflection eye movement system would not contact the roof in rough water.

5. Each front windshield was replaced with a single piece of glass. Originally, these windshields were a two piece unit with vented lower portion (see Figure 2.1).

6. The bow rail was lowered to improve forward visibility. This was necessary because at a normal planing angle the bow rail obstructed much of the horizon. 


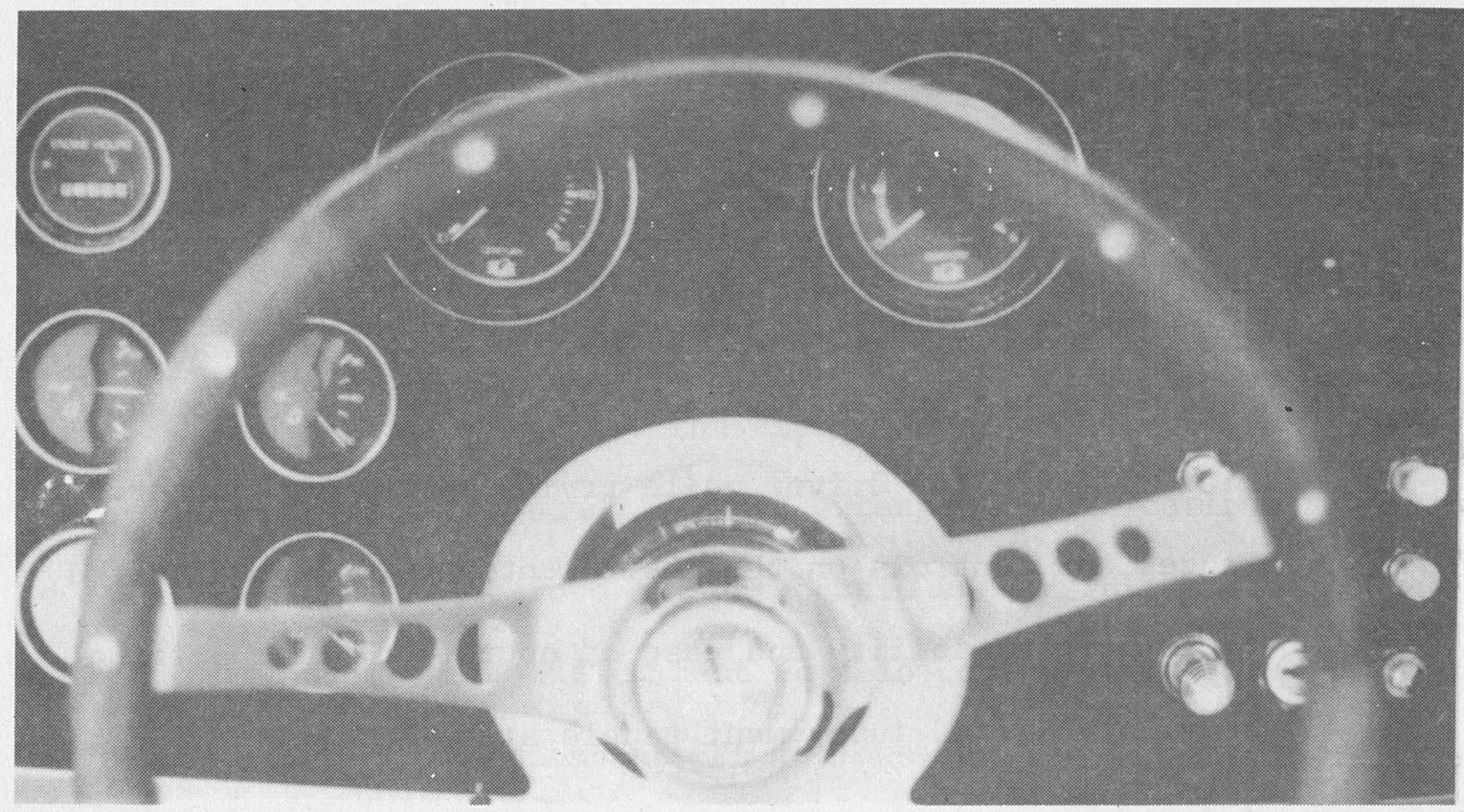

Original Instrument Panel

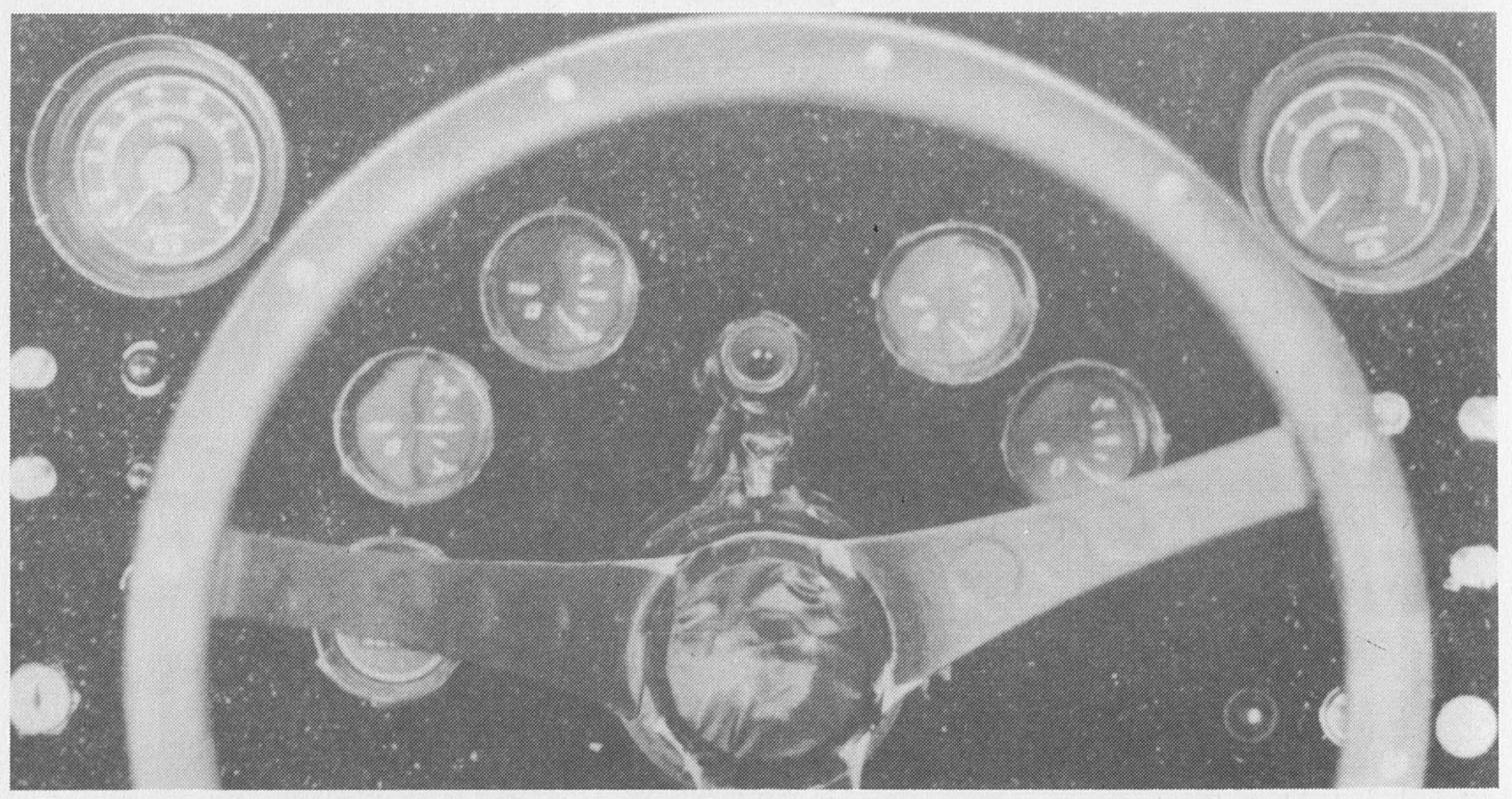

Modified Instrument Panel

Figure 2.3: Original and modified instrument panel 
7. The passenger seat directly behind the driver was removed in order to locate the test equipment electronics as close to the subject as possible without distracting him from his task.

During testing the subject occupied a starboard helm seat with the experimenter occupying a port seat (see Figure 2.4). Behind the experimenter was the camera man who took $35 \mathrm{~mm}$ photographs at various locations along the test route and recorded traffic densities. The equipment monitor was located directly behind the subject.

An electrical modification was made to the boat's engine by adding an auxiliary battery. The two batteries were connected with a battery isolator. Then an inverter drew current off this battery system in order to supply the 120 volts A.C. to the test equipment.

\section{VISUAL ACTIVITY MONITORING SYSTEM}

Numerous apparatus have been developed to record eye fixations. Many of these apparatus used in laboratory settings (e.g., electrooculography and contact lenses) restrict subjects to limited head movements (see Yarbus, 1967 for a discussion of eye fixation/movement recording devices). Automotive eye fixation researchers have usually used portable corneal reflection type recording apparatus.

The corneal reflection eye marker recorder used for this study was developed at the University of Michigan's Industrial and Operations Engineering Department. This "Visual Activity Monitoring" (VAM) system is illustrated in Figures 2.5 and 2.6. 


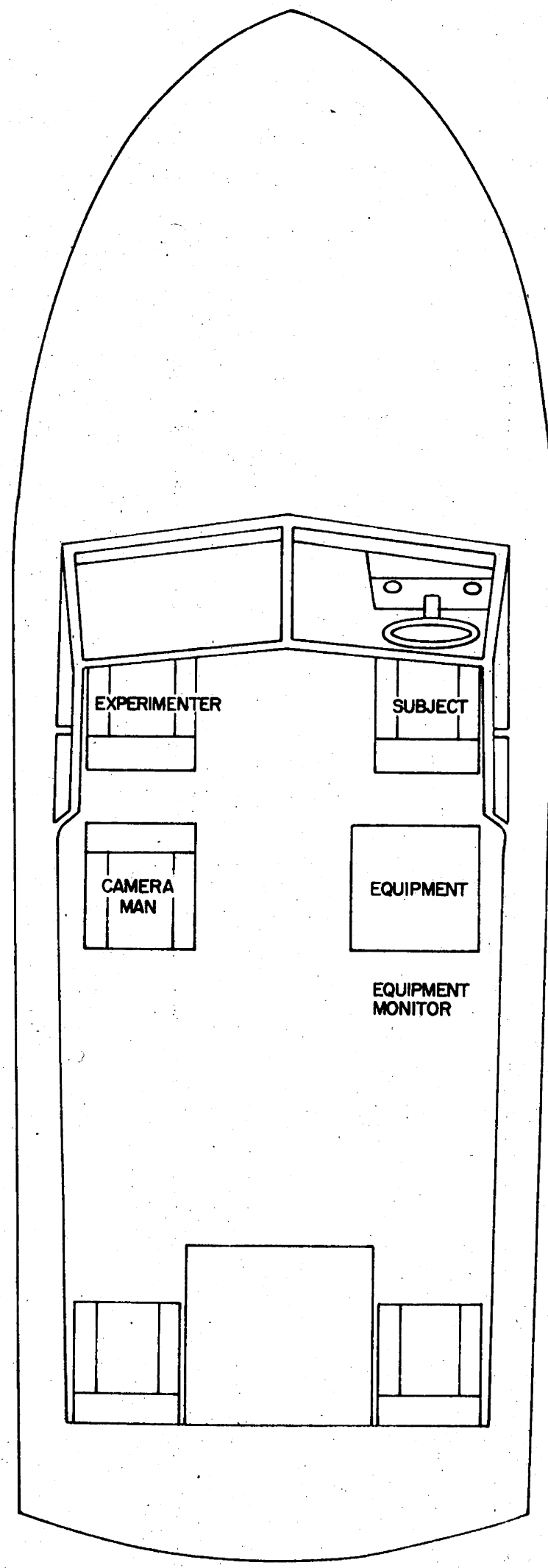

Figure 2.4: Layout of Experimental Boat 


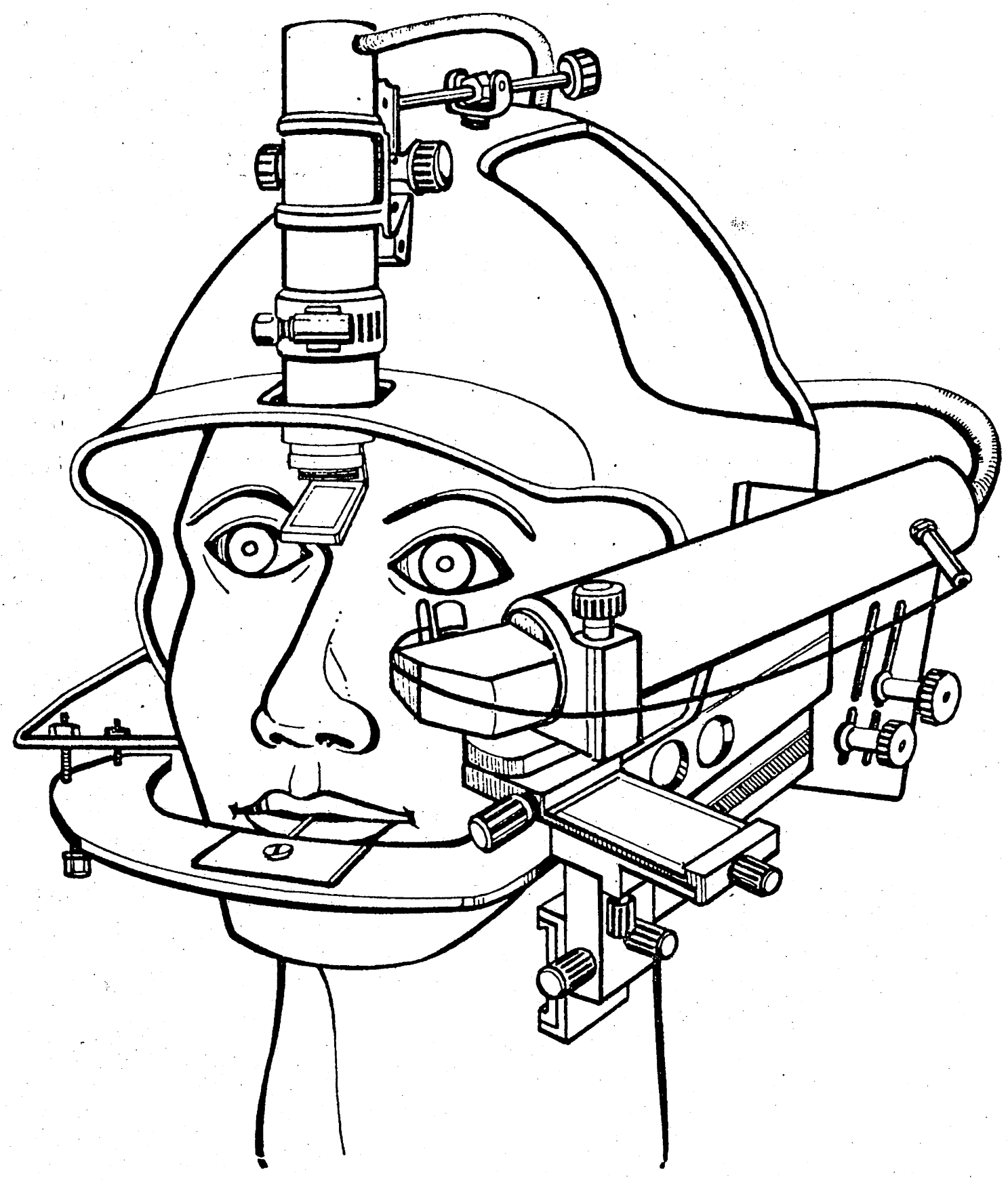

Figure 2.5: Illustration of visual activity monitoring helmet 


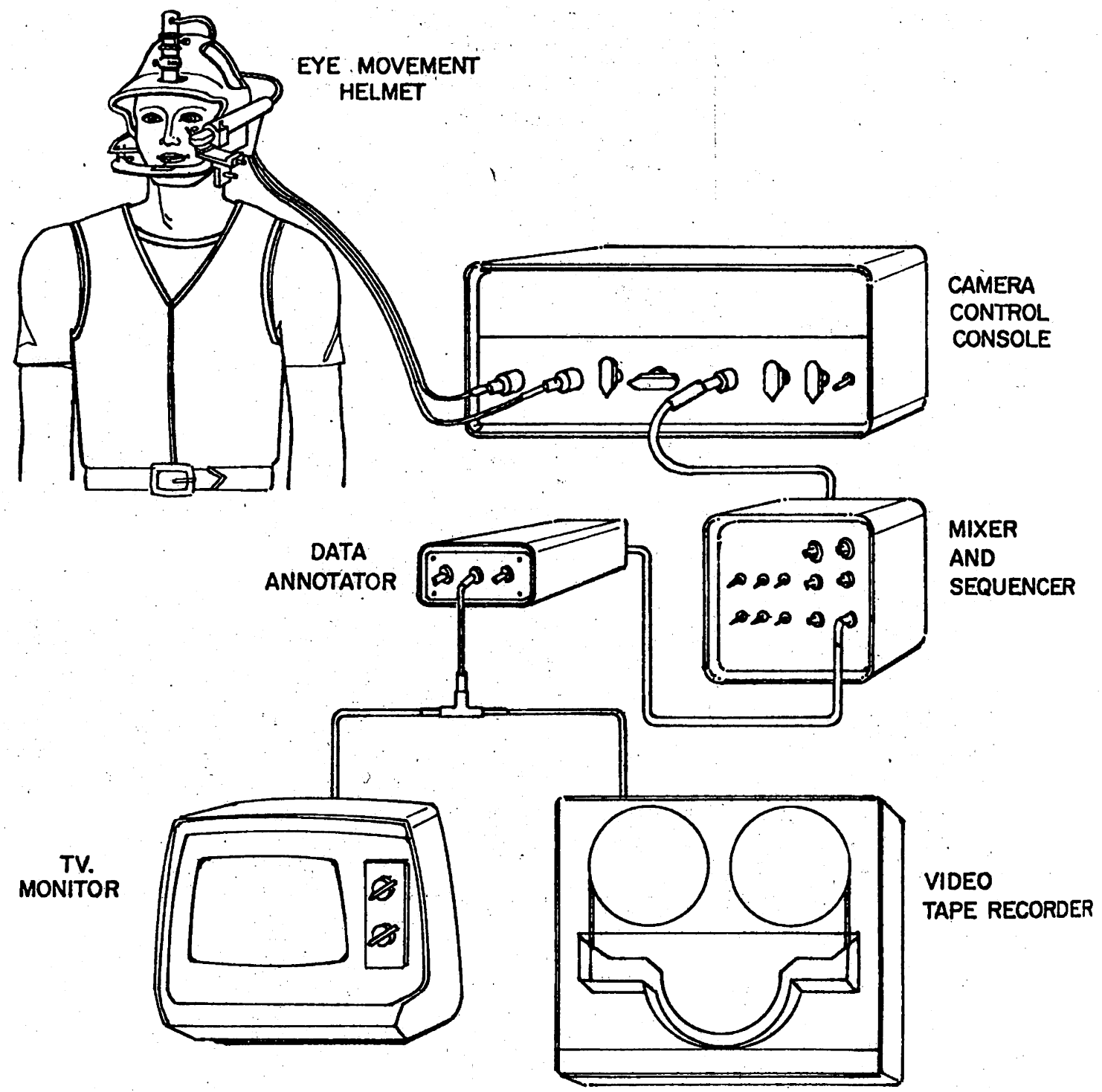

Figure 2.6: Illustration of visual activity monitoring system 
This VAM system was similar in concept to the one discussed by Rockwe11, Bhise, and Mourant (1972). It consisted of a helmet with a custom fitted foam innerliner and stabilized by means of side brackets attached to a bite bar. Television vidicon tubes were mounted on the helmet in front of the left eye to pick up the corneal reflection and vertically on the subject's forehead to record the forward scene. A combination of electronic and mechanical adjustments allowed the corneal reflection image to be superimposed on the field view image. This resulted in a small white dot which was calibrated in such a way as to correspond to the subject's actual viewing location, as illustrated in Figure 2.7.

Pilot tests with the VAM apparatus indicated that the original design had to be modified in order to record data in the boating environment. The following modifications were made:

1. Neutral density filters were added to the head vidicon lens to reduce the amount of light entering the tube.

2. A red light emitting diode (LED) originally used as the corneal reflection light source was neither visible to the experimenters not on the video tape under sunlight boating conditions. Thus, a brighter miniature incandescent lamp was used for the light source.

3. Several ground wires were added to the system.

4. The VAM helmet was painted flat black in order to reduce the glare to the subjects. 


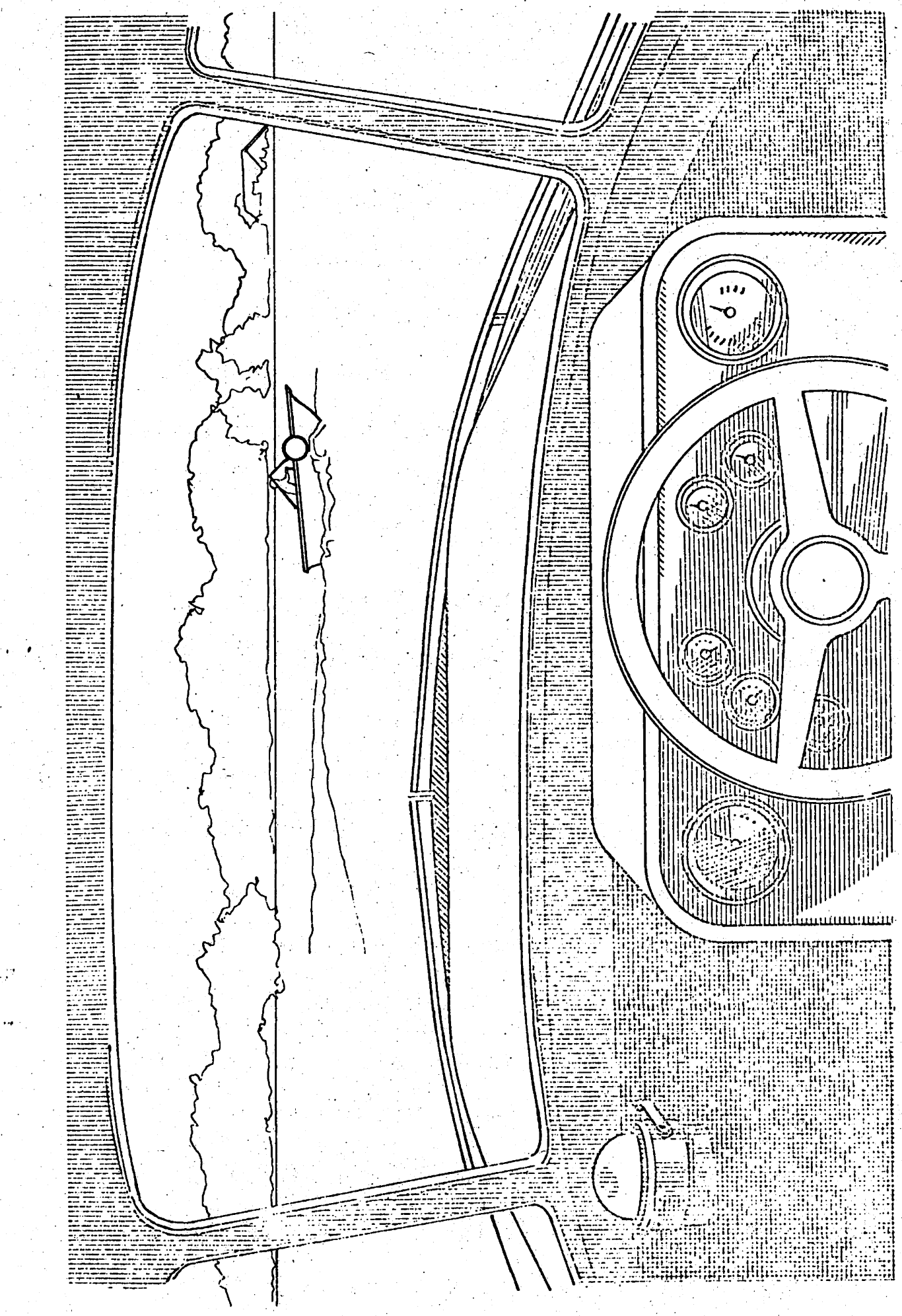

告 
5. The power supply had voltage surges which were related to the engine r.p.m. An adjustable transformer (VARIAC) was added to reduce these surges.

In addition to the above, the auxiliary electronic equipment (e.g., mixer, video tape recorder) was mounted in a plywood cabinet to protect them from the environment.

\section{Eye Marker Calibration}

During the test sessions, calibration adjustment of the system was accomplished using a calibration board which was $2.4 \mathrm{~m}$ ( $8 \mathrm{ft}$. away. This board had horizontal and vertical lines $12 \mathrm{~cm}$ (5 in.) apart and subtended visual angles of $16^{\circ}$ horizontal and $10^{\circ}$ vertical.

To check the calibration at distances other than the $2.4 \mathrm{~m}$ location, the subject fixated on a.) the instrument pane1 gauges and controls which were approximately $.5 \mathrm{~m}$ (20 in.) away from him, b.) external items (such as a point of land, a flagpole) which were at least $100 \mathrm{~m}$ (325 ft.) away, and c.) bow rail markers which were approximately $2.9 \mathrm{~m}$ ( $9 \mathrm{ft}$. ) away. During these calibration sequences the calibration error was considered acceptable if less than $2^{\circ}$.

The initial calibrations for all test runs were recorded on the video film. Periodically through the testing sequence, the calibration was checked by having the subject fixate on certain objects. Minor variations could be corrected electronically. However, if 
larger variations were noted the test was stopped and the system was recalibrated mechanically (with the adjustments available on the helmet). Calibration error was usually caused by the helmet slipping. This occurred because of such things as rough water conditions or the subject trying to "scratch his head." The average callbration error during testing was $1.5^{\circ}$ horizontal and $.7^{\circ}$. vertical. 


\section{CHAPTER III}

\section{RESEARCH METHODOLOGY}

The development of a research methodology for collecting boaters' eye fixation data was one of the major objectives of this research. Another abjective was the evaluation of factors which affect these types of data. In order to have satisfactorily completed these objectives, the factors which were believed to have large affects on boaters' eye fixation were selected as research variables. The selection and implementation of these factors is discussed in this chapter.

\section{INDEPENDENT RESEARCH VARIABLES}

In order to determine differences in boaters' fixation patterns, it was decided to vary their spare capacity using the concept of "attentional demand." Senders, et al., (1967) referred to the "attentional demand" placed on an automobile driver as being a function of 1 ) the roadway, 2) the traffic situation, and 3) the velocity of his vehicle. Translating this concept to boating, the "attentional demand" placed on a boat operator might be a function of 1) the waterway characteristics (boating environment), 2) traffic density, and 3) boat velocity. The type of driving task should probably also be added to Sender's model; and, thus, one would add type of navigation task to the boating analogy. These variables were, thus, considered within the present research as listed in Table 3.1, and how each was involved in the experimental design will now be discussed. 
Tab1e 3.1

Independent Variables

\begin{tabular}{|ll|}
\hline Independent Variable & Levels \\
\hline Navigation Task & Compass \\
& Visual Reference Point \\
& Center in Channel \\
\hline Velocity & Low (29 kmh) \\
& $\begin{array}{l}\text { Medium (42 kmh) } \\
\text { High (56 kmh) } \\
\text { Boating Environment }\end{array}$ \\
\hline Subjects & $\begin{array}{l}\text { Limited Access } \\
\text { Open Water }\end{array}$ \\
\hline
\end{tabular}

\section{Navigation Tasks}

Three navigation tasks were selected as being representative boating tasks. First, a compass task was included in order to replicate the type of task which the subjects were asked to perform in the VAST studies by MacNei11, 'et a1., (1976a). Second, heading the boat to a visual reference point was included since this is one of the most common types of boating navigation tasks. The third 
task, centering in a channel, was similar to the automobile driver's task of keeping his car in the center of a traffic lane.

In addition to being representative, these navigation tasks also controlled the subject's focus of attention. The compass task forced subjects to look inside the boat. The visual reference point task focused attention to a distant point directly in front of the boat, and the channel task focused attention to the external peripheral environment.

A brief description of each task now follows:

1. Compass task: Subjects were instructed to take a $0^{\circ}$ or $180^{\circ}$ heading on a spherical marine compass. These heading were selected because they were the easiest gradient markings to read. The compass task was not a simple task for the subjects since it was constantly oscillating. Thus, subjects were forced to continually monitor it in order to perform the task.

2. External visual reference point task: Subjects were instructed to head the boat to a target such as a water tower or smoke stack which was at least $1.6 \mathrm{~km}(1 \mathrm{mi})$ away. These target objects were selected to be easily visible from a distance because they were high above the shoreline silhouette.

3. Centering in channel task: Subjects were instructed to center the boat in freighter channels marked by buoys. At the narrowest location, these channels were $.3 \mathrm{~km}(.2 \mathrm{mi})$ wide. 
These navigation tasks were structured in order to obtain meaningful results related to boaters' fixation patterns. For example, focusing these power boaters' attention to several different areas provided additional information concerning tasks which were not studied. An example of this is the compass task, which focused the boater's attention inside his boat. This could also be related to a boater preoccupied with something inside his boat, e.g., a passenger, equipment, or some other item which would distract him from his primary task of boating.

\section{Velocity}

The above three navigation tasks were performed at three speeds: 29, 42, and 56 kilometers per hour $(\mathrm{kmh})(18,26$, and $35 \mathrm{mph})$. The minimum speed $(29 \mathrm{kmh})$ was selected as being just above planing with the top speed of $56 \mathrm{kmh}$ chosen as the maximum safe and comfortable speed in choppy water. The intermediate speed, besides allowing for a determination of quadratic velocity effects, approximates a normal, comfortable speed in this. 4.9 to 7.9 meter (16 to 26 foot) boat category.

At the minimum speed of $29 \mathrm{kmh}$, the tests could be best described as boring, the boat was not in an optimal control condition in that more steering movements were required than at the other speeds. Furthermore, this low velocity felt "perceptually slow." The boater should have had more spare capacity at this minimal speed. The medium and high speeds were more characteristic of normal boating speeds in this type of vesse1. 


\section{Boating Environment}

In order to test possible differences due to type of waterway, it was decided to run the test in two different types of boating environments. The first, designated as "limited access water", gave the appearance of being on a medium sized lake. This limited access condition had the following characteristics: a) land was close to the boat, b) it was easy for the driver to determine the location of other vessels in the immediate area, and c) vessels could only enter this area from a few "limited" locations.

The second boating environment was labeled "open water" and gave the appearance of being on a large lake. This large lake environment had the following characteristics: a) land was usually far away from the boat on at least two sides of the vessel, b) it was more difficult to determine the number of boats in the immediate area, and c) boats could approach or enter the area from a multitude of directions.

\section{Subjects}

Prior to selecting subjects, the University of Michigan Medical School Human Use Committee was contacted for approval of the planned research, and this approval was granted.

Subjects were solicited through an advertisement placed in a newspaper which was distributed in the area where the research was to be conducted. Over 40 boaters responded to the advertisement. 
However, a preliminary statistical analysis indicated that the minimum number of subjects to be used for this research should be three. (This analysis is discussed in conjunction with the experimental design.) The three subjects selected met the following criteria:

1. They were experienced boaters who had operated power boats for over five years.

2. They averaged over five hours of boat driving per week during the boating season.

3. They had operated a starboard helm, inboard-outboard drive boat similar to the experimental boat.

4. They were familiar with the test site area.

5. They had normal physical, visual and teeth characteristics. The subjects chosen turned out to be 20-30 years old and had the specific characteristics as 1isted in Appendix A.

\section{EXPERTMENTAL DESIGN}

Prior to developing an experimental design for this research, the preferred number of subjects was determined. An EMS (expected mean squares) table was developed and is contained in Table 3.2. This EMS table determined the tests of significance which would be used in the data analysis. From Table 3.2, it can be seen that the task main effects were tested against the subject-task interaction. In order for the task effects to be significant, the following comparison must hold: 
Table 3.2

EMS Table for Independent Variables ${ }^{1}$

\begin{tabular}{|c|c|c|}
\hline Source & $\begin{array}{l}\text { Degrees } \\
\text { of } \\
\text { Freedom } \\
\end{array}$ & EMS (Expected mean squares) \\
\hline$v_{i}$ & 2 & $6 n \sigma_{V}^{2}+6 \sigma_{S}^{2}+6 \sigma_{V S}{ }^{2}+\sigma_{e}^{2}$ \\
\hline$s_{j}$ & $\mathrm{n}-1$ & $18 \sigma_{S}^{2}+\sigma_{e}^{2}$ \\
\hline $\mathrm{E}_{\mathrm{k}}$ & 1 & $9 n \sigma_{E}^{2}+9 \sigma_{S E}^{2}+\sigma_{e}^{2}$ \\
\hline $\mathrm{T}_{\ell}$ & 2 & $6 n \sigma_{T}^{2}+6 \sigma_{S T}^{2}+\sigma_{e}^{2}$ \\
\hline $\mathrm{VS}_{i j}$ & $2 n-2$ & $6 \sigma_{\mathrm{VS}}^{2}+\sigma_{\mathrm{e}}^{2}$ \\
\hline $\mathrm{VE}_{\text {ik }}$ & 2 & $3 n \sigma_{V E}{ }^{2}+3 \sigma_{S E}{ }^{2}+3 \sigma_{V S E}{ }^{2}+\sigma_{e^{2}}$ \\
\hline $\mathrm{VT}_{i \ell}$ & 4 & $2 n \sigma_{\mathrm{VT}}{ }^{2}+2 \sigma_{\mathrm{ST}}{ }^{2}+2 \sigma_{\mathrm{VST}}{ }^{2}+\sigma_{\mathrm{e}}{ }^{2}$ \\
\hline $\mathrm{SE}_{j k}$ & $n-1$ & $9 \sigma_{\mathrm{SE}}^{2}+\sigma_{\mathrm{e}}^{2}$ \\
\hline $\mathrm{ST}_{\mathrm{j} \ell}$ & $2 n-2$ & $6 \sigma_{\mathrm{ST}}^{2}+\sigma_{\mathrm{e}}^{2}$ \\
\hline $\mathrm{ET}_{\mathrm{k} \ell}$ & 2 & $3 n \sigma_{\mathrm{ET}}{ }^{2}+3 \sigma_{\mathrm{SET}}{ }^{2}+\sigma_{\mathrm{e}}{ }^{2}$ \\
\hline $\mathrm{VSE}_{i j k}$ & $2 n-2$ & $3 \sigma_{\mathrm{VSE}}^{2}+\sigma_{\mathrm{e}}^{2}$ \\
\hline $\mathrm{VST}_{i j \ell}$ & $4 n-4$ & $2 \sigma_{V S T}^{2}+\sigma_{e}^{2}$ \\
\hline $\mathrm{VET}_{i k \ell}$ & 4 & $n \sigma_{\mathrm{VET}}{ }^{2}+\sigma_{\mathrm{SET}}{ }^{2}+\sigma_{\mathrm{VSET}}{ }^{2}+\sigma_{\mathrm{e}}{ }^{2}$ \\
\hline $\mathrm{SET}_{j k \ell}$ & $2 n-2$ & $3 \sigma_{\mathrm{SET}}^{2}+\sigma_{\mathrm{e}^{2}}^{2}$ \\
\hline $\begin{array}{l}\operatorname{VSET}_{i j k \ell} \\
\mathrm{e}_{\mathrm{m}(i j k \ell)}\end{array}$ & $4 n-4$ & $\begin{array}{l}\sigma_{\mathrm{VSET}}{ }^{2}+\sigma_{\mathrm{e}^{2}} \\
\sigma_{\mathrm{e}}{ }^{2}\end{array}$ \\
\hline
\end{tabular}

where: $V_{i}=$ Velocity, $i=1-3 \quad T_{\ell}=$ Navigation Task, $l=1-3$

$s_{j}=$ Subject, $j=1-n \quad e_{m}(i j k \ell)=$ Error, $m=1$

$\mathrm{E}_{\mathrm{k}}=$ Boating Environment, $\mathrm{k}=1,2$

${ }^{1}$ See Hicks (1973) for EMS Table discussion. 


$$
\left.\frac{6 n \sigma_{T}^{2}+6 \sigma_{S T}^{2}+\sigma_{e}^{2}}{6 \sigma_{S T}^{2}+\sigma_{e}^{2}}\right\rangle F_{(3,2 n-2)}
$$

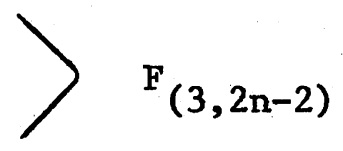

$$
\text { where } \begin{aligned}
n & =\text { number of subjects } \\
T & =\text { task } \\
S T & =\text { subject-task }
\end{aligned}
$$

By assuming that $\sigma_{\mathrm{ST}}$ is zero, this equation can be reduced to:

$$
\left.\sigma_{T}\right\rangle\left(\frac{F(3,2 n-2)^{-1}}{6 n}\right)^{1 / 2} \sigma e
$$

Table 3.3 contains the resultant inequalities for various $n$ (number of subjects). Automotive eye fixation researchers have reported standard errors $\left(\sigma_{e}\right)$ for horizontal location of from $2^{\circ}$ to $4^{\circ}$ (McDowell, 1975). Using this standard error estimate, the greatest "gain" is obtained in going from two to three subjects. Having three subjects appears to be economically beneficial because the gains are smaller in increasing the number beyond three.

The experimental design used for data analysis is contained in Table 3.4 and the mathematical model for this design is of the form: 
Table 3.3

Analysis to Determine Number of Subjects

\begin{tabular}{|c|c|c|}
\hline $\begin{array}{c}\text { Number of } \\
\text { Subjects }\end{array}$ & $\begin{array}{c}\text { For a significant task effect }(\alpha<.05), \\
\text { the following relationship must hold: }\end{array}$ & $\begin{array}{c}\text { If } \sigma_{\mathrm{e}}=4^{\circ} \\
(\text { McDowel1,1975) }\end{array}$ \\
\hline 1 & $\sigma_{\mathrm{T}}>1.2 \sigma_{\mathrm{e}}$ & $\sigma_{\mathrm{T}}>4.8^{\circ}$ \\
\hline 2 & $\sigma_{\mathrm{T}}>.56 \sigma_{\mathrm{e}}$ & $\sigma_{\mathrm{T}}>2.2^{\circ}$ \\
\hline 3 & $\sigma_{\mathrm{T}}>.40 \sigma_{\mathrm{e}}$ & $\sigma_{\mathrm{T}}>1.6^{\circ}$ \\
\hline 4 & $\sigma_{\mathrm{T}}>.32 \sigma_{\mathrm{e}}$ & $\sigma_{\mathrm{T}}>1.2^{\circ}$ \\
\hline 5 & $\sigma_{\mathrm{T}}>.27 \sigma_{\mathrm{e}}$ & $\sigma_{\mathrm{T}}>1.1^{\circ}$ \\
\hline 6 & $\sigma^{\circ}$ & \\
\hline
\end{tabular}

Table 3.4 .

Experimental Design

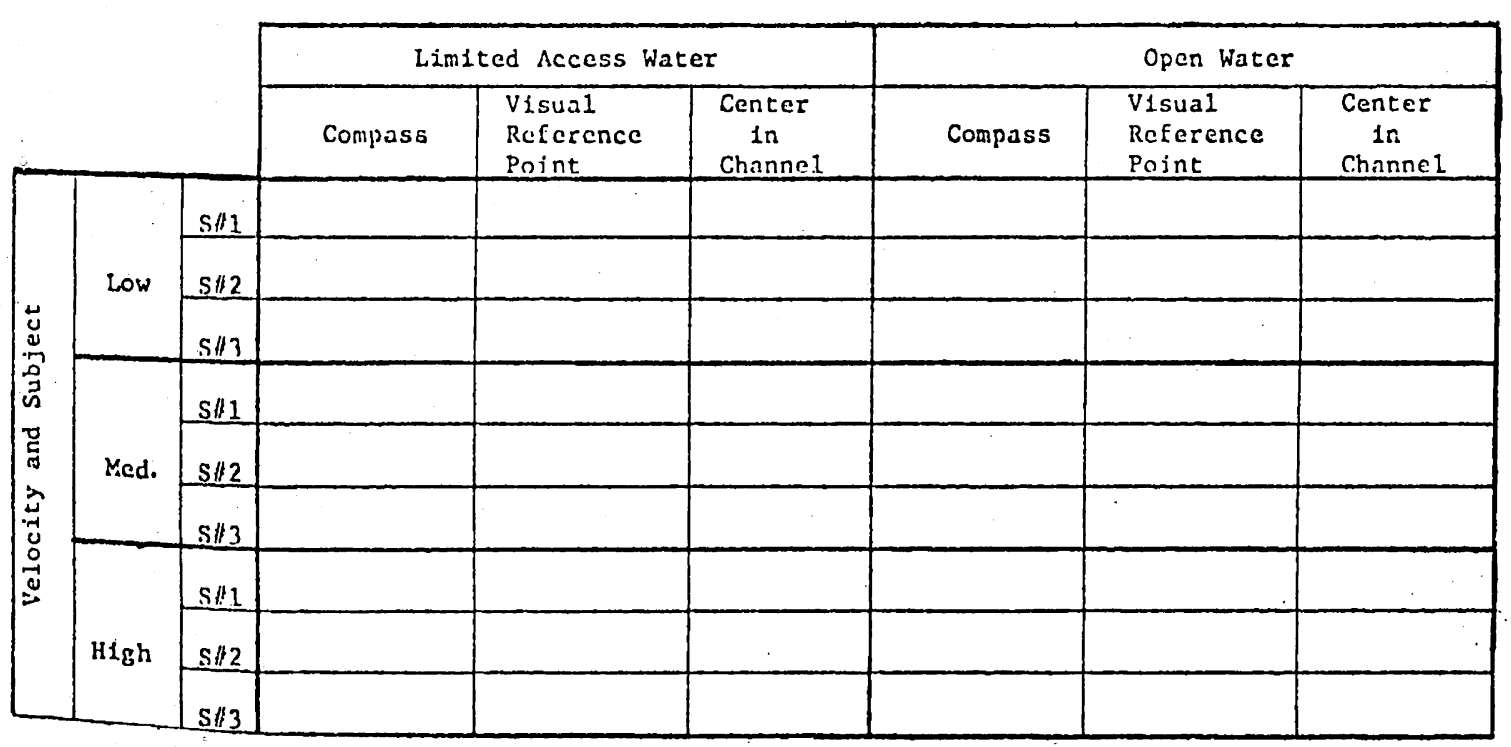




$$
\begin{aligned}
Y_{i j k 1 m} & =\mu+V_{i}+S_{j}+E_{k}+T_{1}+V S_{i j}+V E_{i k}+V T_{i 1}+S E_{j k}+S T_{j 1} \\
& +E T_{k 1}+V S E_{i j k}+V S T_{i j 1}+V E T_{i k 1}+S E T_{j k 1}+V S E T_{i j k 1} \\
& +e_{m(i j k 1)}
\end{aligned}
$$

where: $\quad Y_{i j k l m}=$ Eye fixation parameters (e.g., durations)

$$
\begin{array}{ll}
\mu & =\text { Mean } \\
\mathrm{v}_{i} & =\text { Velocity, } i=1-3 \\
\mathrm{~S}_{j} & =\text { Subject, } j=1-3 \\
\mathrm{E}_{\mathrm{k}} \quad=\text { Boating environment, } \mathrm{k}=1,2 \\
\mathrm{~T}_{1} \quad=\text { Navigation task, } 1=1-3 \\
\mathrm{e}_{\mathrm{m}(\mathrm{ijk} 1)} \quad \text { = Error, } m=1
\end{array}
$$

Observations within this design were randomized with respect to velocity and sequenced through boating environment and navigation task. Use of this factorial design allowed the determination of both the main effects and the interactions. The testing order is discussed in the Test Procedures section.

\section{UNCONTROLLED MEASURED VARIABLES}

In order to insure satisfactory completion of each test run, the data were not collected unless the following conditions were met: 
1. Wave conditions were at a light chop (i.e., not more than 1-2 ft. waves).

2. Weather conditions. were such that a storm would not occur prior to completion of all test segments.

3. Boating traffic was light during data collection, such that not more than one boat was within $.2 \mathrm{~km}$ (250 yds) of the test vesse1.

To insure that the data selected for reduction had light boating traffic, one of the experimenters recorded the moving and anchored boats within the area. For each test segment, this experimenter recorded those boats within $.4 \mathrm{~km}(.25 \mathrm{mi})$ and $\pm 100^{\circ}$ around the subjects' forward vision. The specific categories for which this experimenter recorded observations are listed in Table 3.5.

Other environmental variables, although not controlled during the testing, were recorded at the initiation of each run. These measured environmental variables are listed in Table 3.6.

\section{TEST LOCATION}

The test site used for collecting the data was 1ocated approximately one hour away from Ann Arbor, Michigan. The specific geographical area of the test run was among the islands and lake-1ike bays of the lower Detroit River as it opens into-Lake Erie (see Figure 3.1). This area was ideal for conducting such studies since islands, bays, coastal waters, rivers, and large water type conditions are easily accessible and in close proximity without trailering. 
Table 3.5

Traffic Density Measurements

The following categories of boat traffic were recorded for each test segment:

Overall Traffic Density On

Port*

Starboard*

Moving Boats which Overtook Test Boat On

Port

Starboard

Test Boat Overtook Other Boats

Moving on Port

Moving on Starboard

Anchored on Port

Anchored on Starboard

Head-On Approaches to Other Boats Which Were

Port

Starboard

Other Boats Crossed Test Boat's Path

From Port

From Starboard

$*$ Port $=$ left, Starboard $=$ right

Table 3.6

Environmental Conditions Recorded for Each Test Run

The following items were recorded from the Detroit Weather Report:

1. Sky (e.g., cloudy, partly sunny)

2. Temperature

3. Humidity

4. Wind Speed

5. Wind Direction

6. Barometer Reading

The following items were recorded by direct observation by one of the experimenters:

1. Percent Cloud Cover

2. Weather Conditions (e.g., cloudless, overcast, rain, etc.)

3. Water Conditions (e.g., calm, choppy, etc.)

4. Wave Height

5. Visibility (in miles)

6. Visibility (i.e., good, fair and poor)

7. Wind Condition (e.g., none, moderate, etc.)

8. Wind Direction 


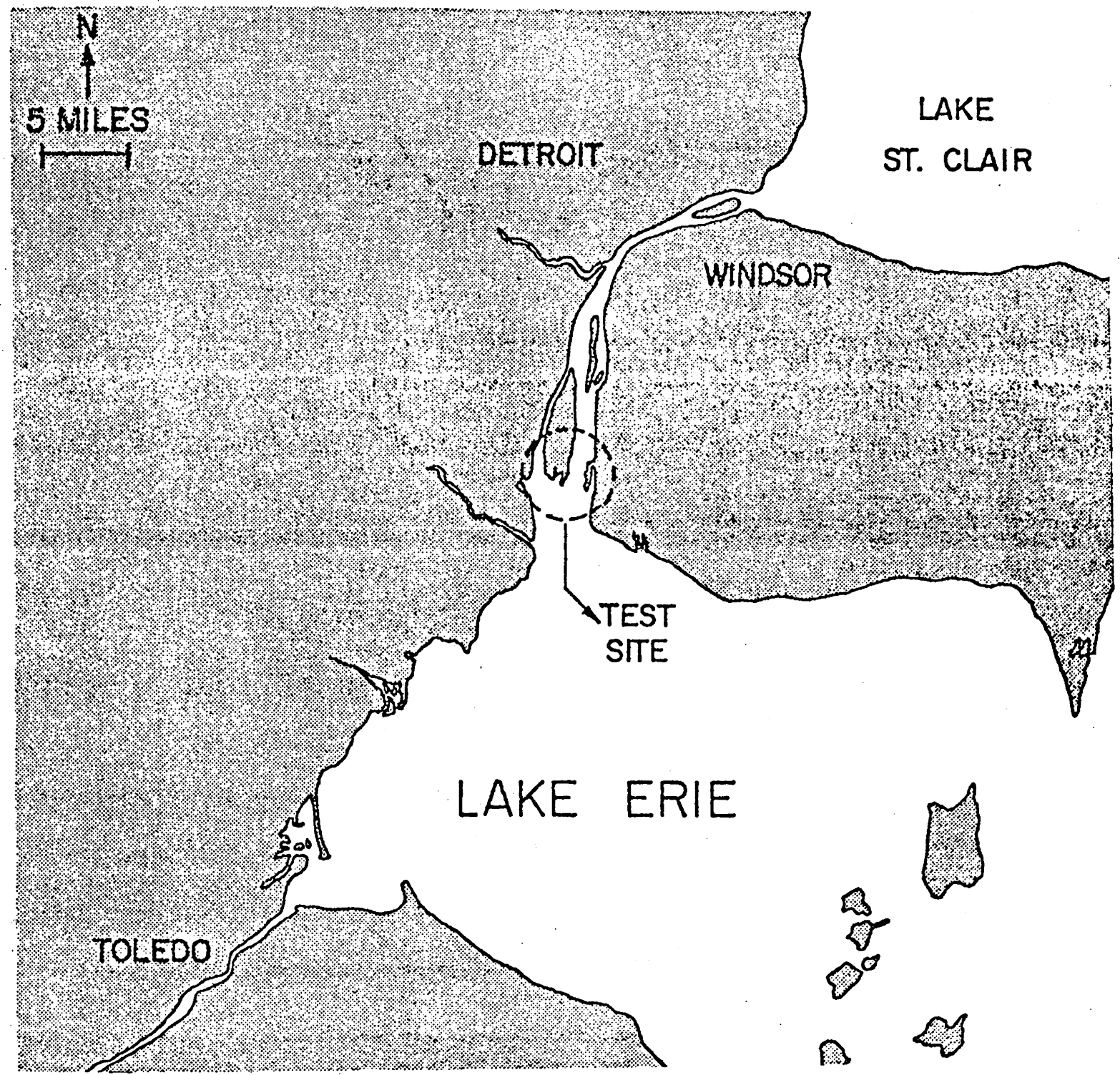

Figure 3.1: Test site location 
An overall view of the test route selected is illustrated in Figure 3.2. The limited access water conditions, which are in the lower half of this figure, are further magnified in Figure 3.3; while the open water conditions are in the upper half of Figure 3.2 and magnified in Figure 3.4. This test route proved to be a very interesting and non-monotonous course which satisfied the following:

1. During the compass task, it permitted a compass heading which prevented the boater from using an external reference point instead of using the compass. (It would not been easier for the boater to head the boat toward a tall tree or other distinguishable environmental factors than to follow a compass heading.)

2. Conditions were varied enough such that subjects could not memorize the traffic in the locality.

3. In the open water condition, the land was far enough away such that the boater appeared to be on a large inland lake.

4. The route was compact enough to minimize the test time.

5. At least one minute of data could be collected after the subject was performing the specific naviagtion task at the desired test speed.

In choosing the limited access water condition shown in Figure 3.3, land was always within .2 kilometers. This appeared to the subject as a medium sized lake environment where the boater was cruising and the shoreline was fairly close to his vessel. In the open water environment (Figure 3.4) 1and was always at least 


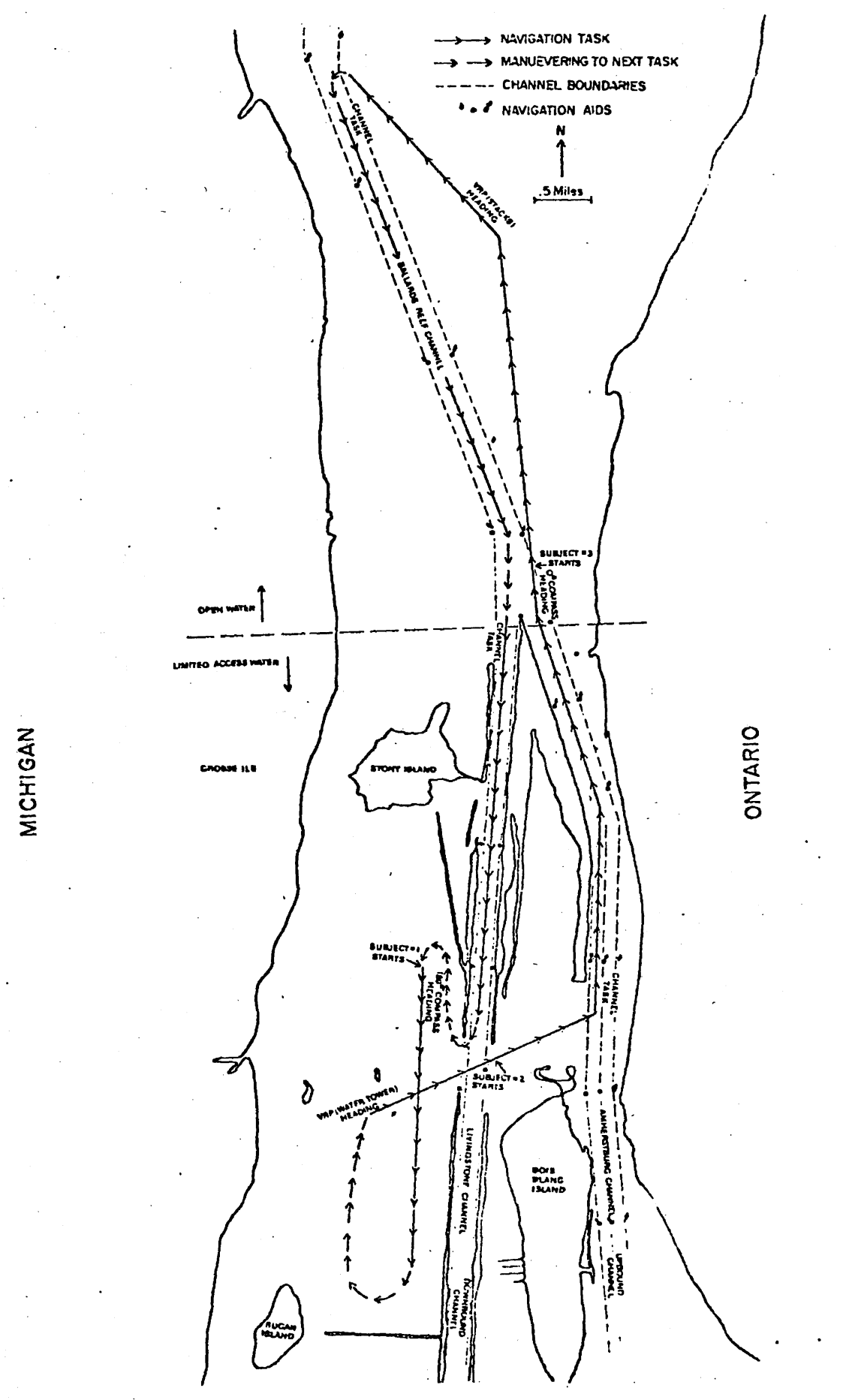

Figure 3.2: Boating test course 


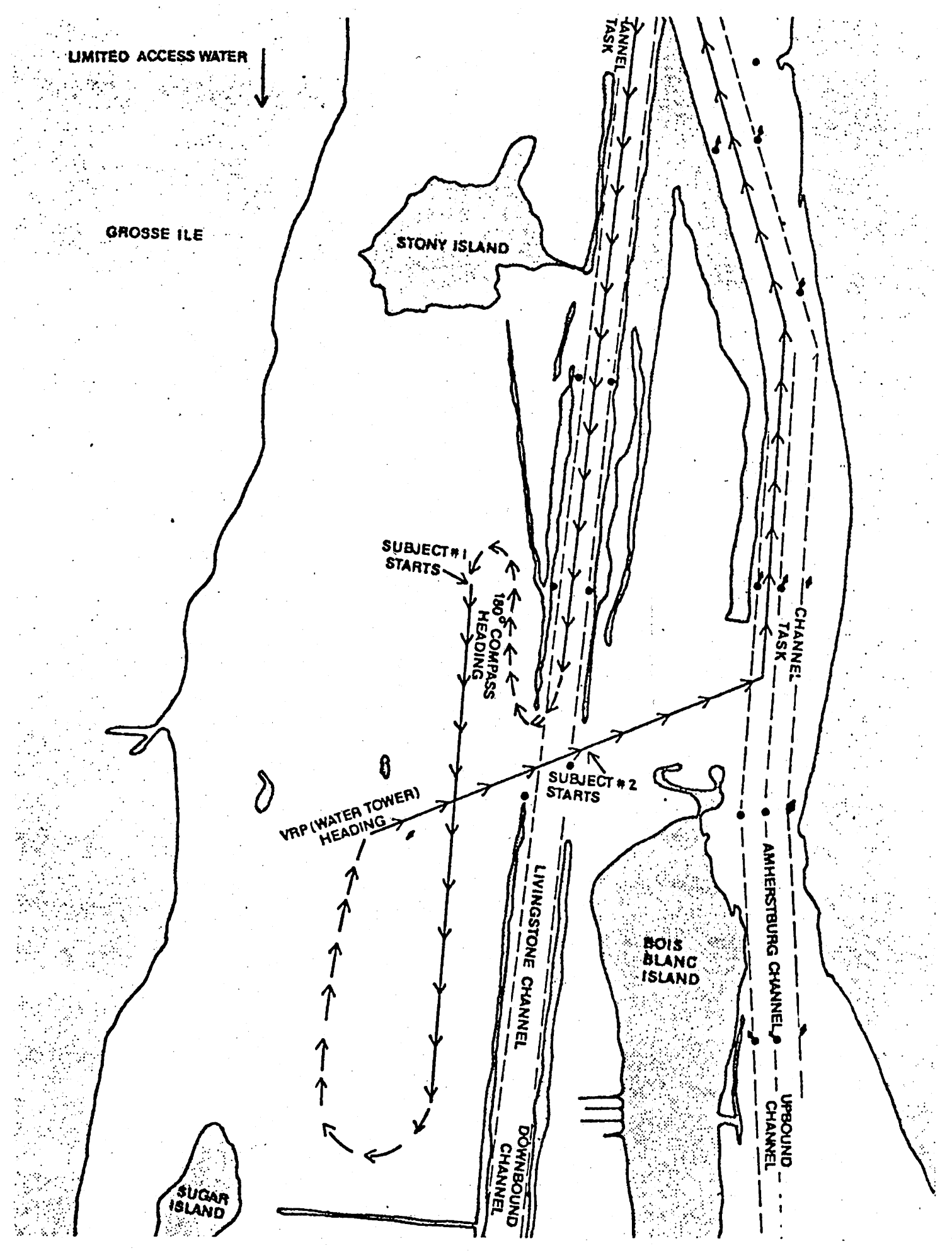

Figure 3.3: Limited access boating test course 


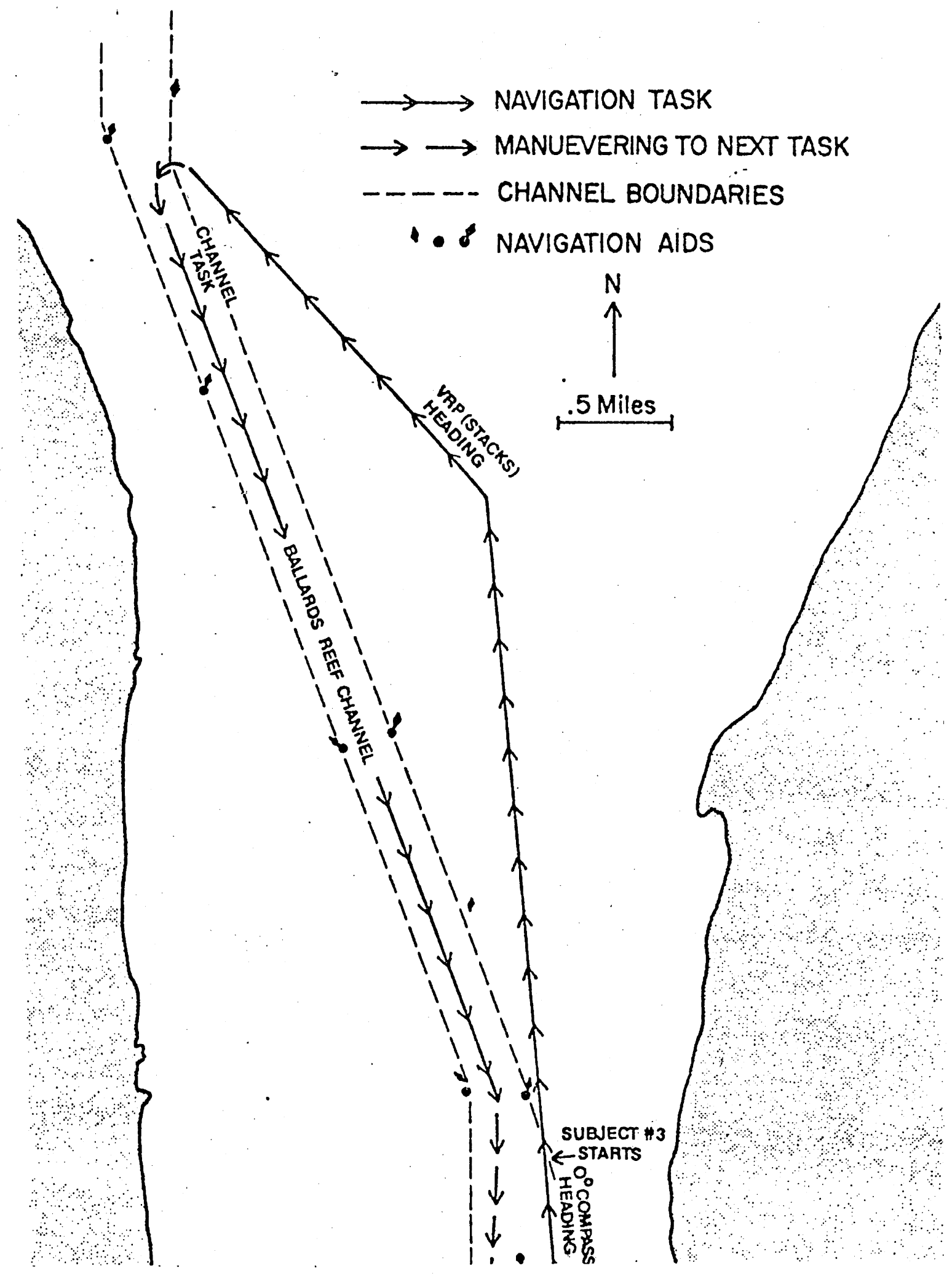

Figure 3.4: Open water boating test course 
.6 kilometers away from the test boat. This gave to the boater the appearance of being on a large lake since a great expanse of water was either in front of or behind the test vessel.

As mentioned earlier, subjects sequenced through the several Navigation Tasks and Boating Environments within the experimental design cells. This was necessary to conserve time. As an example from Figure 3.4, it would not be possible to finish the channel task and proceed to the reference point task without wasting precious minutes of nonfruitful data collection. To offset this sequencing effect, subjects started at different locations as illustrated in Figure 3.2.

Due to the length of the test segments, it was only possible to perform at most two velocity levels during each of these segments. This required subjects to maneuver through the test course twice. In order to assign the sequential order to the segments to be performed, it was, first, decided randomly whether one or two velocity levels would be performed for each subject and each navigation task on the first run through the test course. Second, corresponding velocity levels were then randomly assigned. As an example, Subject $\|_{1}$, who started in the limited access compass task, performed at the low velocity. He then progressed to the limited access, visual reference point task and performed this at the low then the medium velocity levels. The ordering for a11 test sequences is contained in Appendix A. 
Traffic densities were very light during the week on this test course. The channels marked in Figure 3.2 are freighter channels and occasionally a freighter was encountered at a safe distance during testing. Specific details of the test phases will now be discussed.

\section{TEST PROCEDURES}

\section{Pre-Test Subject Preparation Phase}

During the subject's first visit to the base facilities, he was familiarized with the test vessel and controls; he viewed a video tape explaining the type of data recorded for the study; and he signed a consent form before proceeding with other activities. A complete list of all data collection activities for all phases is contained in Table 3.7.

In previewing the test boat, the subject was permitted to enter the boat and sit in the driver's. seat and was shown the various instrument panel displays and controls. This included a demonstration of the single level throttle-gear shift selector and the function of the switches on the instrument pane1. Any questions that the subject may have posed were answered; however, all subjects seemed to be generally familiar with the types of controls and layout of the cockpit.

After this introduction to the boat, the subject was taken into the base facility to preview a video tape which showed what the eye movement system helmet looked like on a subject and the type of data which were to be collected. Further details of the study were then explained to the subject and he was asked if he was still willing 
Table 3.8

Data Collection Test Day Events

\begin{tabular}{|c|c|}
\hline Day & Events \\
\hline 1 & $\begin{array}{l}\text { The following activities were performed: } \\
\text { 1. Initial viewing of test boat } \\
\text { 2. Explanation of study and signing of Implied } \\
\text { 3. Vonsent Form } \\
\text { 4. Anthropometric Measurements taken } \\
\text { 5. Dental Bite Bar molded } \\
\text { 6. Foam Headliner constructed } \\
\text { 7. Initial piloting of boat by subject }\end{array}$ \\
\hline 2 & $\begin{array}{l}\text { 1. Fitting of VAM Helmet } \\
\text { 2. Piloting of boat by subject with helmet } \\
\text { 3. Calibration of VAM System }\end{array}$ \\
\hline 3 & $\begin{array}{l}\text { 1. Calibration of VAM System } \\
\text { 2. Piloting of boat by subject with helmet }\end{array}$ \\
\hline 4 & 1. Data collected for Coast Guard Study \\
\hline 5 & 1. Data collected for Dissertation \\
\hline
\end{tabular}

to participate in the test sessions. More specific details of the study such as the number of hours and the pay were explained; and then, he was asked to voluntarily sign the subject consent form contained in Appendix A.

A vision test was given using a Bausch and Lomb Ortho-Rater. This measured characteristics such as subject's acuity, color vision, 
aphoria and depth perception. Using a yardstick and tape measure, anthropometric dimensions were then taken, and these included measurements relevant to the boat"s seating arrangement. Subjects! vision and anthropometric measurements are contained in Appendix A. The Visual Activity Monitoring system required a very secure fit on each subject's head in order to maintain the stability. Thus, a foam innerliner and a dental bite bar were customly fabricated for each subject. The dental bite bar was made by warming a metal form which was covered with dental impression wax (Kerr impression compound, type 1, red). This was inserted into the subject's mouth such that it came in contact with his upper and lower teeth. The subject bit into this impression material and maintained pressure for approximately one minute until it had hardened.

The head foam innerliner required a carefully executed procedure. Basically, it is made from pressurized foam ingredients injected into a mold which was placed on the subject's head and hardened in approximately three minutes. Precautions were taken to reduce any discomfort that the subject might feel during this foaming procedure and no subjects complained of being uncomfortable.

After completing the bite-bar and helmet liner fabrication, the experimenters took the subject for his first famillarization run in the test vessel. The objectives of this run were to acquaint the subject with the operating handling characteristics of the vessel and the visual landmarks In the specific test area. One of the experimenters explained the functions of the cockpit controls to the 
subject, went through the engine starting checklist, started the boat, and maneuvered it away from the dock area. Once the test vessel was maneuvered away from a residential area and also other boat traffic, the subject was permitted to take over the operation of the boat.

In this familiarization run, subjects were given maneuvering instructions as to the turns to make with the boat, changes in speed using the tachometer and any specific compass headings they were to maintain. As these maneuvers were performed, two of the experimenters subjectively evaluated the boater's skill on a scale of 1 to 10 , by making judgments about certain boating situations and his handling of the vessel. A number 5 would represent an average boater, a number 10 would be the most skilled, professional type boater. All subjects in this study performed at the 5 to 7 range as judged by the experimenters. Thus, one could classify the subjects as being average to slight1y above average in boating skil1. This familiarization run took approximately 45 minutes, after which the subject could ask any further questions. A time was then arranged for him to return for his second test session.

\section{Familiarization Phase}

As noted in Table 3.7, upon arrival for his second session, the subject was briefly fitted with the entire VAM system and a corneal reflection eye spot was obtained. This was done in the test station and not on the boat. Upon its completion, the subject was taken 
to the boat after removing the helmet. In the boat, the helmet was again placed on the subject's head and stabilized with the bite bar system. Since the object of this second test session was for the subject to become familiar with driving the boat while wearing the VAM system, the corneal reflection was not obtained and no data were recorded.

After one of the experimenters backed the boat out of the dock area, the subject was permitted to take over the controls for the balance of the run. He then proceeded through the test site area but not through the specific test course. The run took approximately one hour and by the end of the run, all subjects seemed to be performing normally and were familiar with the landmarks of the test site area. Upon completion of this run, each subject was then rescheduled for a third test session.

The original schedule called for data to be collected during the third test session. Unfortunately, electrical problems with the VAM system arose during this session. Thus, subjects were given an extra day for additional familiarization with the boat and VAM system.

\section{Coast Guard Data Collection Phase}

A fourth day test session was scheduled which took approximately six hours. During this time, data were collected to fulfill a Coast Guard contract. This contract studied the effect of traffic density, velocity and fatigue on boater's eye movement patterns and 
details of tt are available from a report by Miller, Gatchell and Dykstra (1977). During this test session, the subject drove the test vessel through a prescribed course very similar to that which he had driven through on his familiarization days. Each subject went through the test course three different times with an approximate one hour rest period between each run. Corneal reflection eye movement data were collected on the first and third runs while only head movement data were collected on the second run. Head movement data were obtained from a third vidicon tube mounted above the instrument panel in such a location that a facial view of the subject could be obtained (see Dykstra, 197.7), After completion of this test session, the subject was scheduled to return for his fifth test session which is described below.

\section{Experimental Data Collection Phase}

Upon arrival for his fifth test session; the subject entered the boat and adjusted the driver's seat to a comfortable location. The VAM helmet was then placed on the subject's head, stabilized and the corneal reflection was located. Once the experimenters were satisfied that the equipment was functioning, it was removed from the subject's head and he drove the boat to a calm area near the beginning of the test run. Again, the equipment was placed on the subject's head. Calibration was then accomplished using a portable grid system which was positioned in the rear of the boat and by having the subject fixate on distant reference points, on bow markers 
located at the front of the boat, and on specific instruments in the instrument panel. Once calibration was completed, the subject , proceeded to the test course illustrated in Figure 3.2. As previous1y stated, each boater started at a different point in the course and went through the total route twice (see Appendix A for test sequence). Checks on calibration were repeatedly made during the test session when data were not being collected. The total run lasted approximately one hour, which seemed to be the approximate time until the helmet system began being uncomfortable.

Chapters II and III presented evidence to satisfy objective 非 (development of a research methodology and establishment of the feasibility of collecting boaters' eye fixation data). The following chapter will now analyze the effect of several chosen factors on boaters' eye fixations (Objective 非). 


\section{CHAPTER IV}

\section{RESULTS}

This chapter addresses Objective $\$ 2$ of this study and is divided into two major sections. The first section discusses the data reduction techniques which were employed prior to analyses in order to manipulate the raw data. The second section contains the empirical analyses performed on these data.

\section{DATA REDUCTION}

To insure homogeneity of the data sets selected for reduction, criteria were established to aid in the selection process (see Table 4.1). After determining the sequences to be reduced, the spatial and temporal parameters of the eye fixations within each sequence were determined.

A frame by frame analysis was performed to determine these parameters of the data. To facilitate this type of manual reduction, a slow motion, stop action video tape recorder was utilized along with a television monitor. Superimposed on the TV monitor was a clear acetate grid vertically and horizontally divided into $2^{\circ}$ intervals with a resultant range of $20^{\circ}$ in both axes. This grid was utilized to determine the distance in degrees a given eye spot was from a particular reference point. The two lines which determined the $(0,0)$ reference point were the horizon and a vertical boat marker, (see Figure 4.1). 
Table 4.1

Criteria for Data Selected for Reduction

The data selected for reduction also had to satisfy a set of criteria as follows:

1. The operator had to be performing the selected navigation task at the correct velocity and had to be heading the boat in a straight 1ine. (The auditory portion of the video tape was useful, since the experimenter could be heard giving the subject navigation commands and any velocity changes could be detected.)

2. Low traffic density conditions of no more than one moving boat within 275 meters had to exist.

3. A complete segment consisting of 64 distinct in-view fixations had to be available. This number was arrived at by determining the maximum number of fixations which could be reduced from all segments of data. This resulted in segments being, on the average, 40 seconds long. (Coincidentaily, this 40 second data segment length was used by Steinman (1976). He also stated that Ditchburn and Foley-Fisher had proposed this length (40 seconds) be adopted as an international standard for eye movement research. 


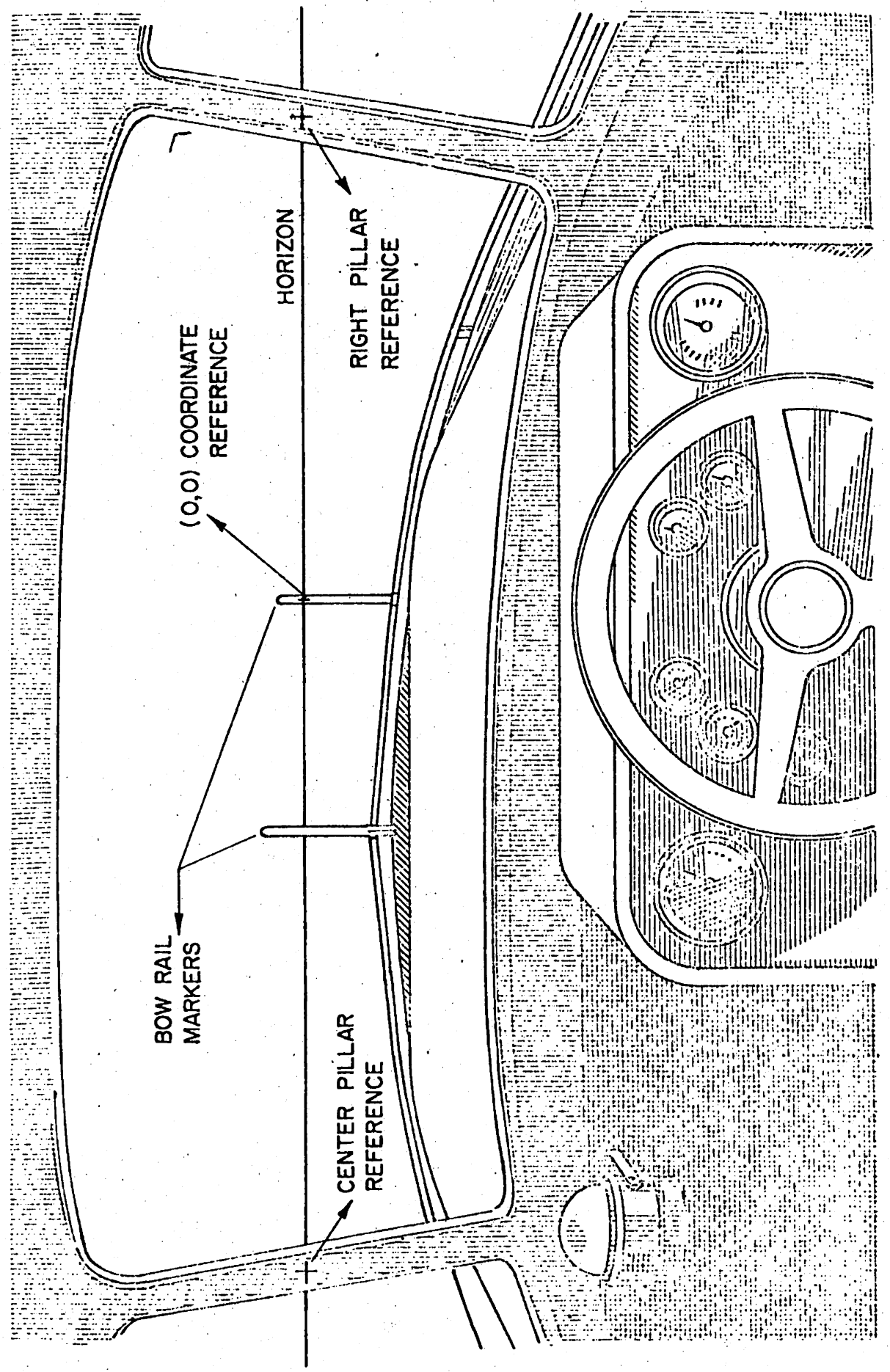

 
If the bow rail marker was not visible in the TV picture (e.g., if the subject turned his head to the side) then one of the boat pillars was used as a reference line. U1timately, all spatial coordinates were re-referenced to the horizon and the vertical bow marker 1ocated directly in front of the driver.

After determining the spatial and temporal fixation parameters within a particular sequence, the data reducer again viewed the sequence. During this second viewing, other task related information was categorized. All variables determined by the data reducer are listed in Table 4.2 and detalled explanations are contained in Appendix B.

Upon initial viewing of the data tapes, a problem was encountered relating to the definition of a fixation, as will now be discussed.

\section{Fixation Definition}

A problem arises in defining a new fixation when the distance between fixations is small, on the order of a few degrees. While very critical to eye movement research, there has been no agreed upon method for defining a new fixation. Moreover, researchers are usually vague about specifying the criteria they used for defining these fixations. Rutley and Mace (1968) counted the number of eye movements subjects made which exceeded $5^{\circ}$. Their criterion number is extremely large since Rockwell (1971) stated that most eye fixations in automobile driving were less than $6^{\circ}$ in travel distance. 


\section{Table 4.2}

Dependent Measures Recorded by Data Reducer

The following dependent measures were determined for each data sequence: ${ }^{1}$

Immediate Boating Situation (e.g., another boat is approaching port)

Maneuver (e.g., subject is moving the vessel straight through light choppy water)

Traffic Density, Moving (number of moving boats within $1 / 2 \mathrm{mi}$ )

Traffic Density, Anchored (number of anchored boats within $1 / 2 \mathrm{mi}$ ).

Reference Location (the reference for the eye spot coordinates)

Beginning Digitizer Number (where there is no eye spot movement)

Ending Digitizer Number (where there is no eye spot movement)

Horizontal Coordinates of Eye Spot (with respect to the reference point)

Vertical Coordinates of Eye Spot (with respect to the reference point)

Calibration Error, both Horizontally and Vertically

Fixation Target (e.g., subject is fixating on a moving boat)

${ }^{1}$ see Appendix B for detailed categories of dependent measures 
Lambert, et a1., (1974) discussed a computer system of data reduction which incorporated a complex set of criteria for determining a new fixation.

The determination of these new fixation durations strongly depend on the instructions given to the data reducer. Difficulties arise because of drifts and involuntary microsaccades which can accompany fixations. The longer the fixation duration, the higher the chance of observing these drifts or involuntary saccades (Yarbus, 1967). Yarbus illustrated that drifts and involuntary saccades were as large as $1 / 2^{\circ}$ and the durations were usually from $300-800 \mathrm{msec}$. Many types of eye movement recording systems (e.g., suction cap devices and Purkinje image methods) are capable of determining these drifts and involuntary saccades. The corneal reflection eye movement systems are usually poor at determining these micro eye movements. Within this current study a precise criteria definition of a new fixation was determined by the data reducer who used the criteria in Table 4.3. These and all the dependent measures as 1isted in Table 4.2, were entered into the computer. The following section discusses the various transformations made on the raw data.

\section{Fixation Location Determination}

The computer programs used to transform the data were taken from the Michigan Interactive Data Analysis System (MIDAS) as developed by Fox and Guire (1973). Basically, these MIDAS programs were necessary to re-reference the fixation data, test for normality, determine 
Table 4.3

Criteria for Defining a Fixation

The following criteria were used to define a new fixation:

1. Spatial travel distance was greater than $1^{\circ}$ (This eliminated drifts and Involuntary saccades which occurred and were less than $1^{\circ}$ )

2. The beginning of a fixation was the first frame when the eye spot was stationary after making a transition.

3. Fixations had to be longer than three frames ( $50 \mathrm{msec}$ ). (Although Lambert, et a1., (1974) used a $100 \mathrm{msec}$ duration criteria, others (Gould, 1976 and Carpenter and Just, 1976) have noted durations as short as $50 \mathrm{msec}$.

4. The end of a fixation was the last frame where the eye spot was stationary and not blurred as in making a saccade to a new fixation.

statistical parameters such as means and standard deviations, and develop prediction equations. These specific manipulations on the raw data will now be discussed.

The data reducer determined the calibration error (i.e., in azimuth and elevation degrees) for a particular sequence by viewing the calibration check just prior to and just after that particular 
sequence. This error was noted for each fixation and incorporated into the computer program which determined the re-referenced relative spatial coordinates of each fixation.

These computer programs, besides taking into account the calibration error, also adjusted the eye spot coordinates with respect to the original reference point. If the eye spot was initially referenced to a location not straight ahead, then its coordinates were re-adjusted. This was accomplished by determining the angle from straight ahead for each auxiliary reference point on the boat and each particular subject. It was necessary to make this determination for each subject since their different statures and seating positions significantly affected the angular location to these references as viewed from their eye location.

\section{Fixations Eliminated from Data Sets}

Inftial analyses of the resulting data indicated strong biases due to the navigation tasks. Clearly, the navigation tasks were selected to force changes in the boater's focus of attention as mea-

sured by his spatial coordinates. As a result, the spatial coordinates and the duration measures exhibited trends that could be explained by the strong biases due to the navigation tasks.

The strongest of these biases was exhibited during the compass task. The compass was mounted at $-40^{\circ}$ azimuth, $-10^{\circ}$ elevation. Thus, fixations to this instrument strongly affected the means and standard 
deviations of the resultant data sets. Figure 4.2 illustrates the bimodal distribution which results for horizontal fixation locations during the compass task. Removing those fixations which were on the compass resulted in only $2 \%$ of the fixations being located at $-40^{\circ}$ rather than the $16 \%$ as illustrated in Figure 4.2 .

Fixation durations were also strongly biased due to the compass fixations. Average overall fixation durations ranged from $260-530 \mathrm{msec}$, while the specific compass durations averaged $1150 \mathrm{msec}$.

The initial analysis of the results using all fixations in the data sets did result in many significant effects. However, it was difficult to distinguish between those effects primarily caused by the biases as mentioned above, and those that were truly related to the boaters' "normal" fixation patterns. Since one of the objectives of this research was to determine boaters! normal visual patterns, the navigation task fixations were removed from the data sets. Thus, fixations to the compass were removed from the compass data sets, fixations to the water tower or smoke stacks were removed from the visual reference point data sets, and fixations to the channel markers or buoys were removed from the centering in channel data sets. A1though the fixations to the compass had the greatest biasing effect on their respective data sets, fixations to the visual reference points or channel markers had a noticeable effect on their data sets. Thus, all fixations specific to a given navigation task were removed from the data sets ultimately analyzed. 

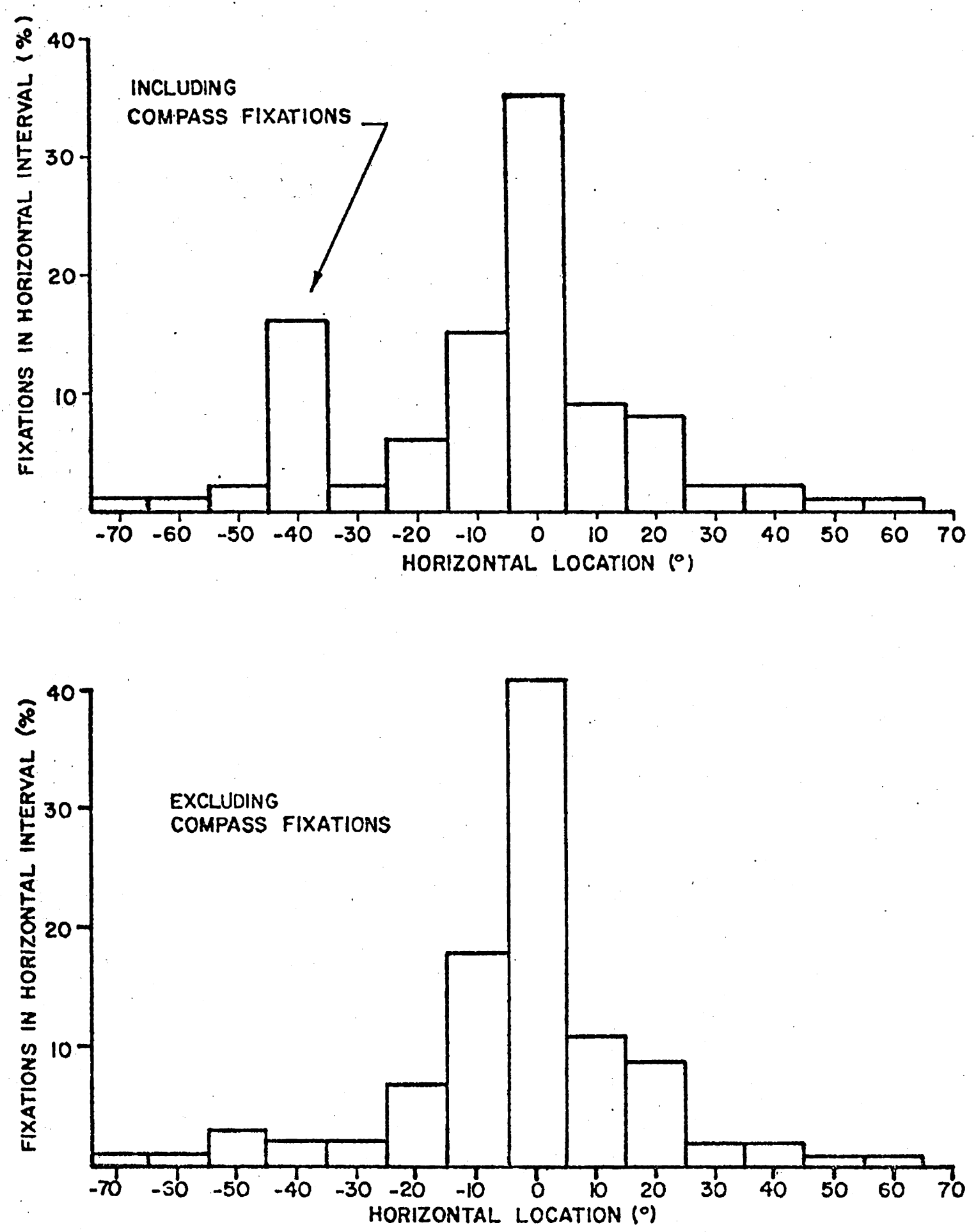

Figure 4.2: Histograms of horizontal location including and excluding compass fixations (data from compass navigation tasks) 
Another item of concern when analyzing the boaters' spatial patterns had to do with traffic density. As previously mentioned, the data sets analyzed contained only the low traffic density situation, (not more than one boat being present at any particular moment). However, this still resulted in an inconsistency throughout the data sets. As an example, a boat could have been approaching from the right, from the left, or there could have been no traffic at all within the different data sets. Furthermore, the speed with which another boat approached probably had an effect on the number of fixations the operator made on it. Thus, it was decided to further remove from the data sets fixations to all other boats (either moving or anchored). Although this did not seem to greatly affect the resultant dependent measures, it did delete some outlying spatial fixations in a few of the data sets.

After removing fixations specific to navigation tasks and othèr boats, the resultant data sets encompassed what this author believes to be "normal" boaters' fixation patterns in non-vehicular avoidance situations (i.e., monitoring for obstacles in his path),

\section{ANALYSIS OF RESULTS}

A list of all the dependent measures which are discussed in the following sections is contained in Table 4.4. The following analysis explores those dependent measures which are of importance when discussing eye fixation patterns. Of particular interest are the spatial and duration characteristics of the fixations. 
Table 4.4

Dependent Eye Fixation Variables Analyzed

Spatial Scanning Patterns

Horizontal and Vertical Fixation Locations.

Eye Spot Trave1 Distances

Temporal Scanning Characteristics.

Fixation Durations

Visual Zone Fixation Percentages (See Appendix D)

Fixation Targets

Prediction of Horizontal and Vertical Fixation Locations and Fixation Durations (See Appendix E)

The following analyses of the data utilized a full factorial, statistical model with subjects as random effects, and with all the non-significant mean squares pooled to determine significant effects. (The equation for this model was presented on p. 42.)

ANOVA's were computed from the resultant data sets using the Biomechanical Computer Program for analysis of variance BMD8V (Dixon, 1974). These analyses will now be discussed.

\section{Spatial Patterns - Horizontal and Vertical Fixation Locations}

The horizontal and vertical fixation location results illustrated in this section are portrayed with mean \pm 1 standard deviation ellipses. These ellipses assume a bivariate normal distribution and because there 
was no correlation between the horizontal and vertical components, their slope is zero. These types of ellipses are a convenient method for displaying a large amount of information concerning fixation locations; however, they have been used only once before in the eye movement 1iterature (Bhise and Rockwe11, 1971).

Statistical analysis of the vertical fixation location components revealed no significant effects due to any of the independent research parameters. Thus, further discussions in this section include only those effects related to the analysis of the horizontal component of eye fixations. (Although the ellipses illustrate both components.)

As illustrated by the centering in channel tasks in Figure 4.3, the horizontal fixation location parameters were normally distrlbuted. Analysis indicated that for these distributions the skewness was about -.3 and the kurtosis was about 3.2 as determined by methods described by Hahn and Shapiro; (1967).

Performance of the ANOVA routines on the various data sets required that means and standard deviations be calculated (Appendix C). A sumary of the significant effects from the resultant ANOVA's of horizontal location are contained in Table 4.5. These results will now be discussed.

\section{Navigation Task Effects}

As illustrated in Figure 4.4, the significant effects which are in the "Task" column of Table 4.5 occur because boaters scanned a greater area foveally while performing the visual reference point task, 


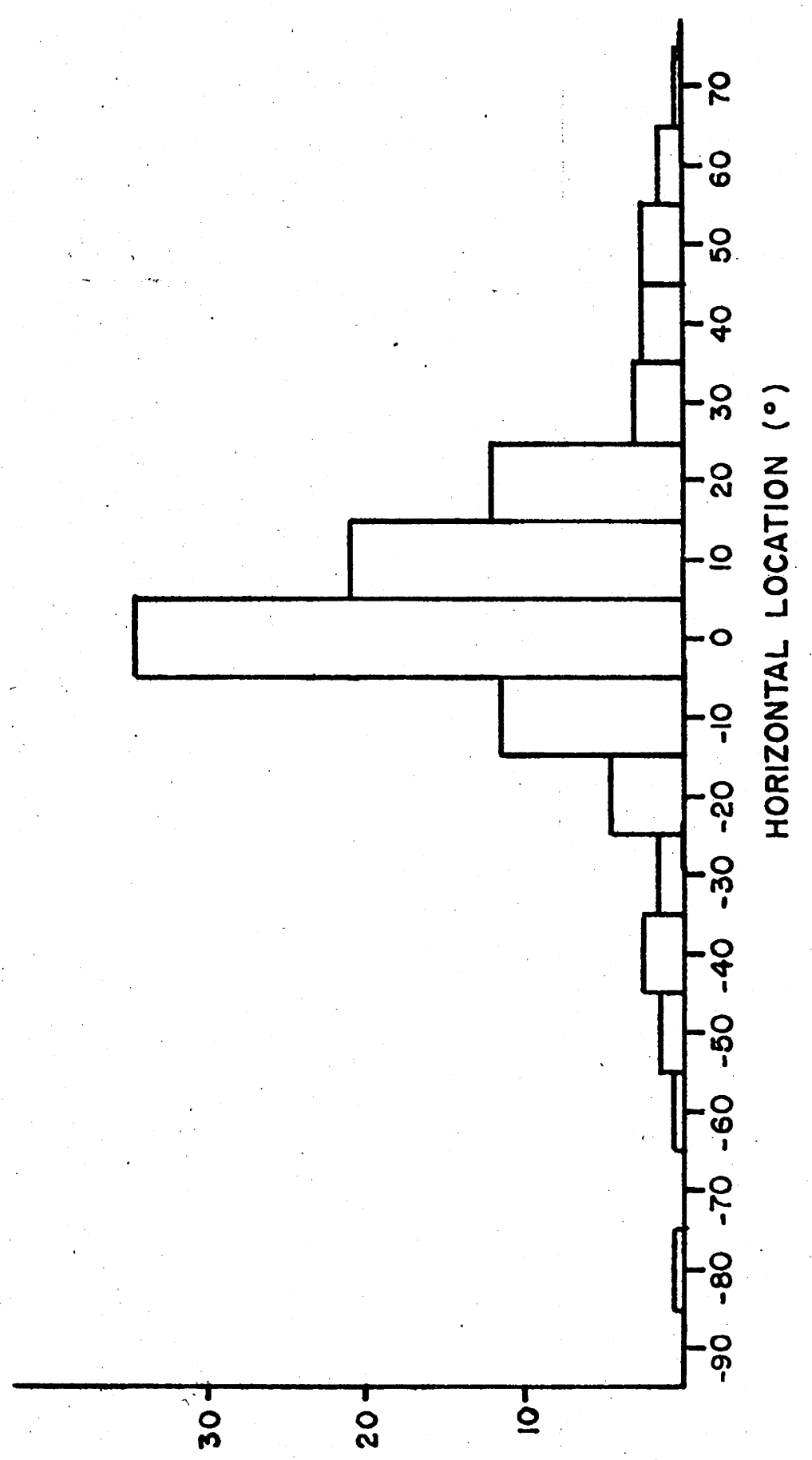

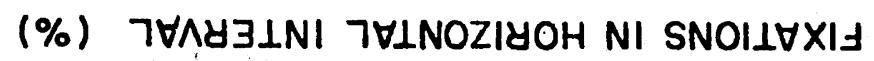

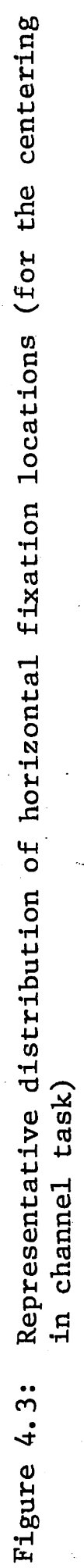


Table 4.5

Significant Results from the Analysis of

Variance for Horizontal Location

of Eye Fixations 1

\begin{tabular}{|l|l|l|l|l|l|l|l|}
\cline { 2 - 6 } \multicolumn{1}{c|}{} & \multicolumn{6}{c|}{ Independent Variables } \\
\hline $\begin{array}{l}\text { Dependent } \\
\text { Variable }\end{array}$ & $\mathrm{V}$ & $\mathrm{S}$ & $\mathrm{E}$ & $\mathrm{T}$ & $\mathrm{SE}$ & $\mathrm{ET}$ & $\mathrm{SET}$ \\
\hline $\begin{array}{l}\text { Horizontai } \\
\text { Location: } \\
\text { Mean } \\
\text { Standard } \\
\text { Deviation }\end{array}$ & $*$ & & & & & & \\
\hline
\end{tabular}

${ }^{1}$ Data sets contain only those fixations not on a particular navigation target or other boats.

where: $V=$ Velocity

$S=$ Subject

$\mathrm{E}=$ Boating Environment

$\mathrm{T}=$ Navigation Task

$$
\begin{aligned}
& * \quad=\alpha<.05 \\
& * * \quad=\alpha<.01 \\
& * * *=\alpha<.005 \\
& * * * *=\alpha<.001
\end{aligned}
$$

than while they were performing either the compass or channel tasks. Furthermore, during this visual reference point task, their mean horizontal location was almost straight ahead while in the other two tasks it was $3^{\circ}$ from straight ahead. (The compass task mean horizontal location was $-3^{\circ}$, while the centering in channel task mean locations was $\left.+3^{\circ}\right)$. Figure 4.4 also 11lustrates that during the channel task, boaters scanned almost the same area to the right of straight ahead as during the visual reference task and scanned less area to the left. During the compass task they scanned a similar area to the left as during the visual reference task but less area to the right. 

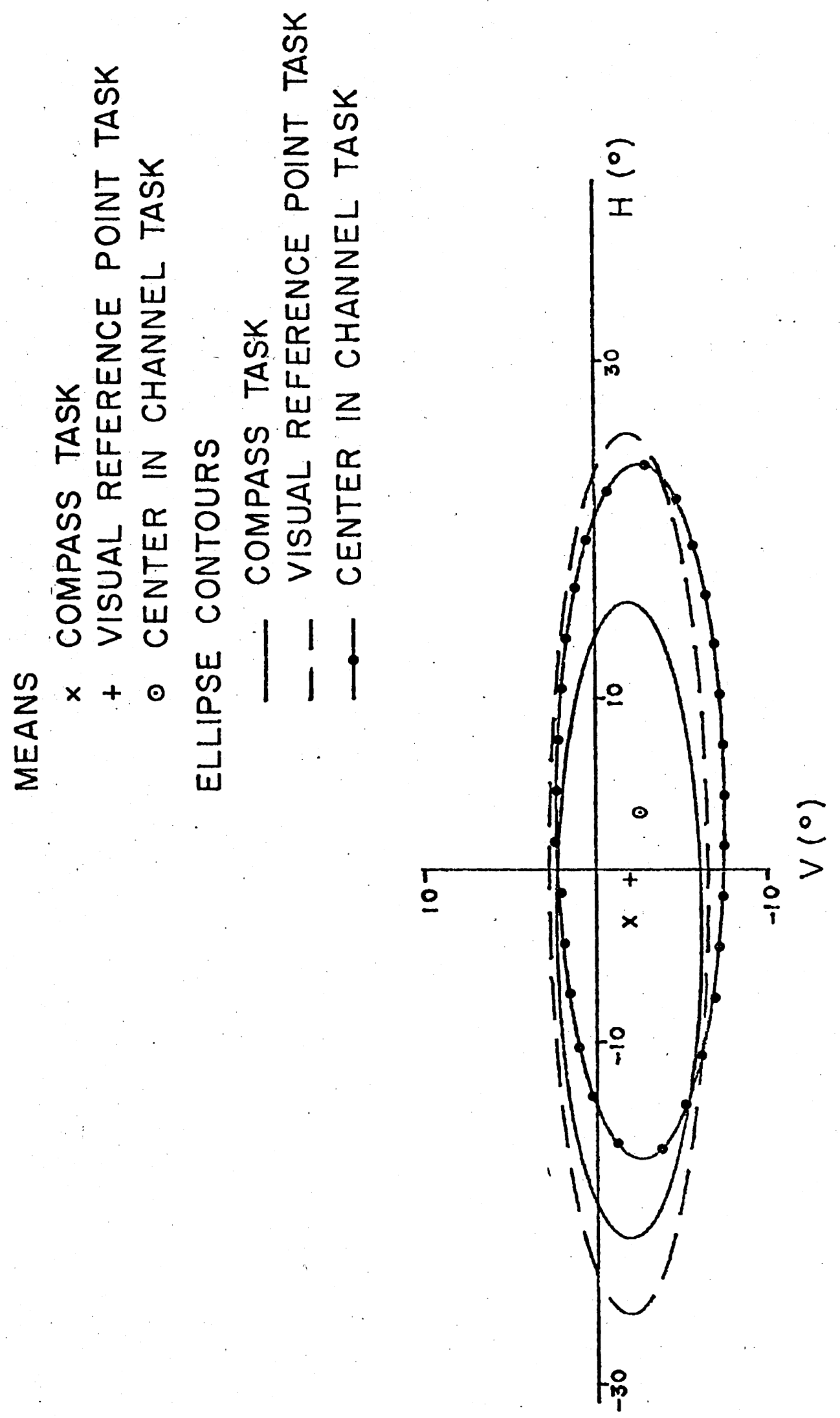

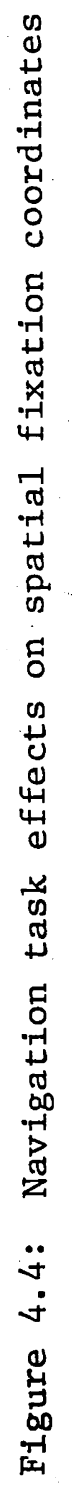


Boating Environment Effects

Table 4.5 also shows that changes in eye movement patterns were significant due to the boating environment of the test. In an unexpected result, the boaters scanned a larger area in the limited access environment than in the open water (see Figure 4,5 ). In the open water environment, boaters scanned almost the same area to the left of straight ahead; however, they scanned $10^{\circ}$ less to the right of straight ahead.

\section{Subject Effects}

The subject column (S) of Table 4.5 indicates significant differences for the standard deviation of horizontal location. This result is illustrated in Figure 4.6. Subject $\left.\right|_{2}$ had a smaller scanning area (standard deviation was $10^{\circ}$ less than either subjects 非 or 非3). Throughout much of the analyses, Subject 非 had numerous differences from Subjects $\|_{1}$ and 3 .

\section{Subject-Boating Environment Effects}

This subject effect was further magnified by the subject-boating environment interaction in column "SE" of Table 4.5. This is nicely illustrated in Figure 4.7. In the limited access water condition, the scan patterns for Subjects $\|_{1}$ and 3 were similar while subject \#2 scanned a smaller area. During the open water condition, subject \#2's pattern was similar to that of Subjects 11 and 33 to the right 

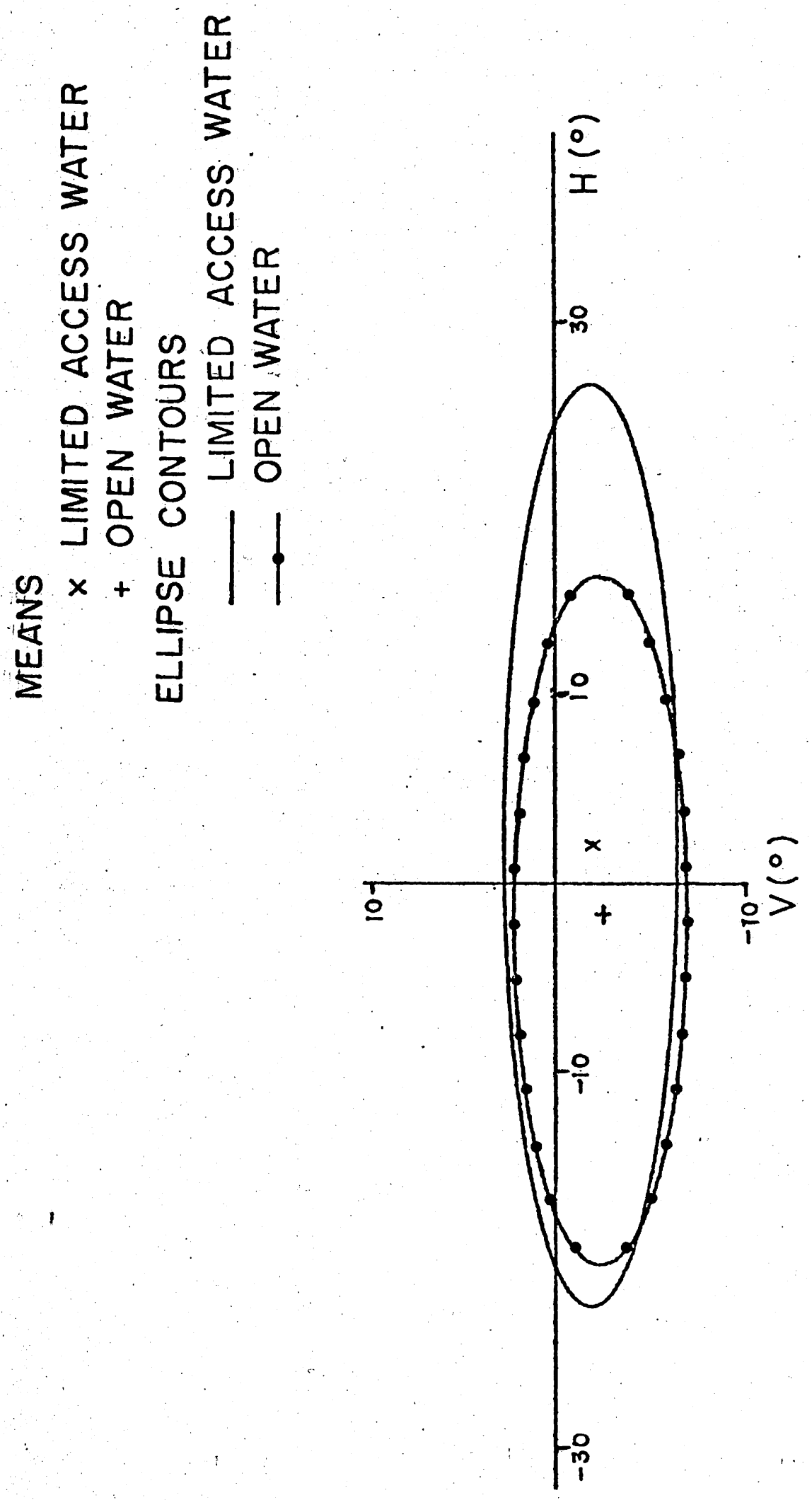

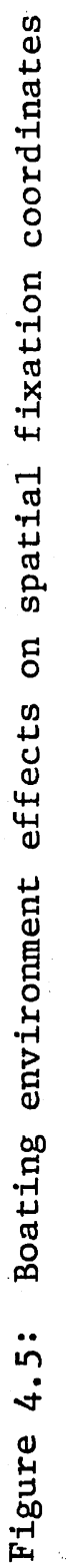



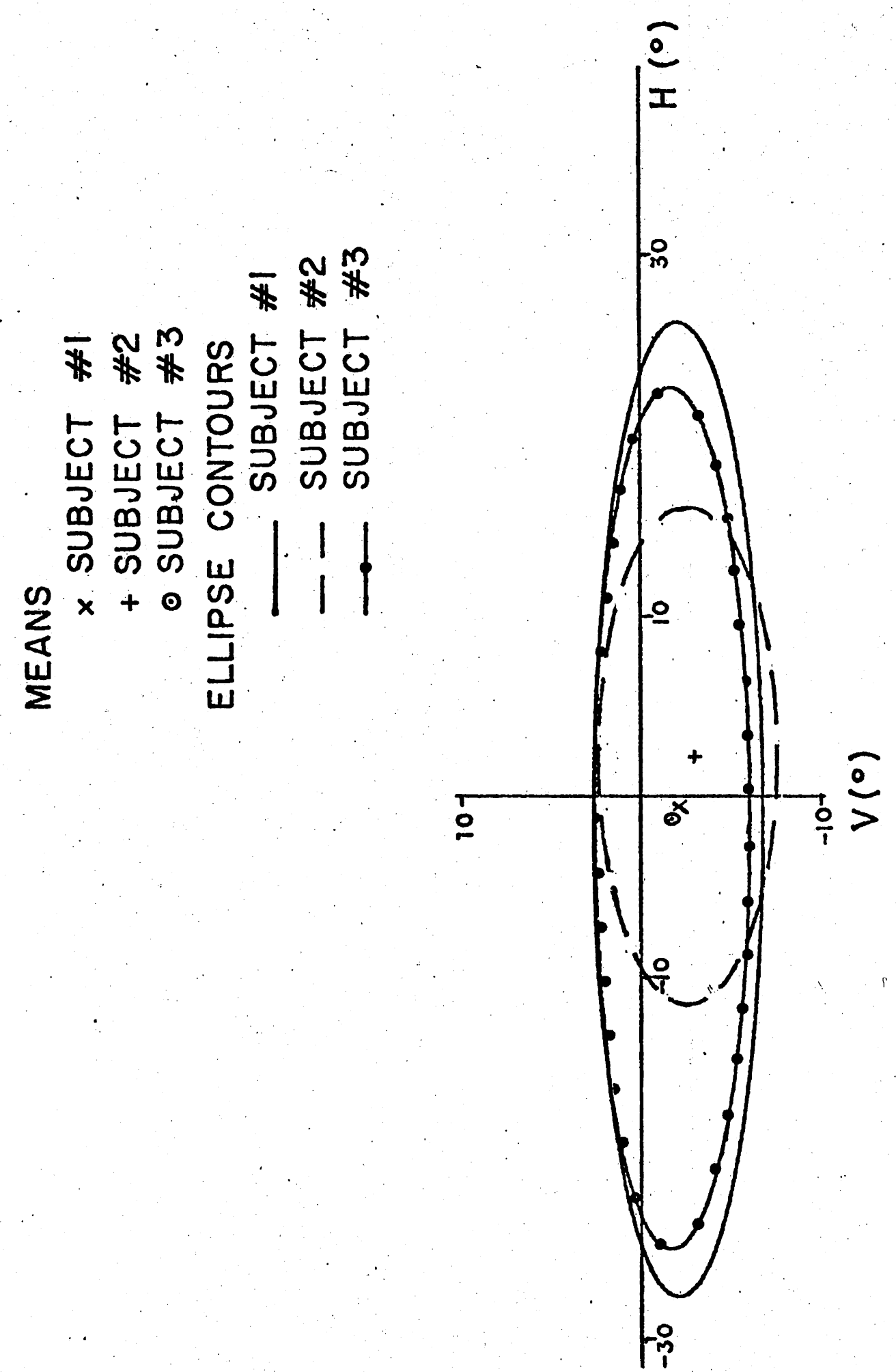


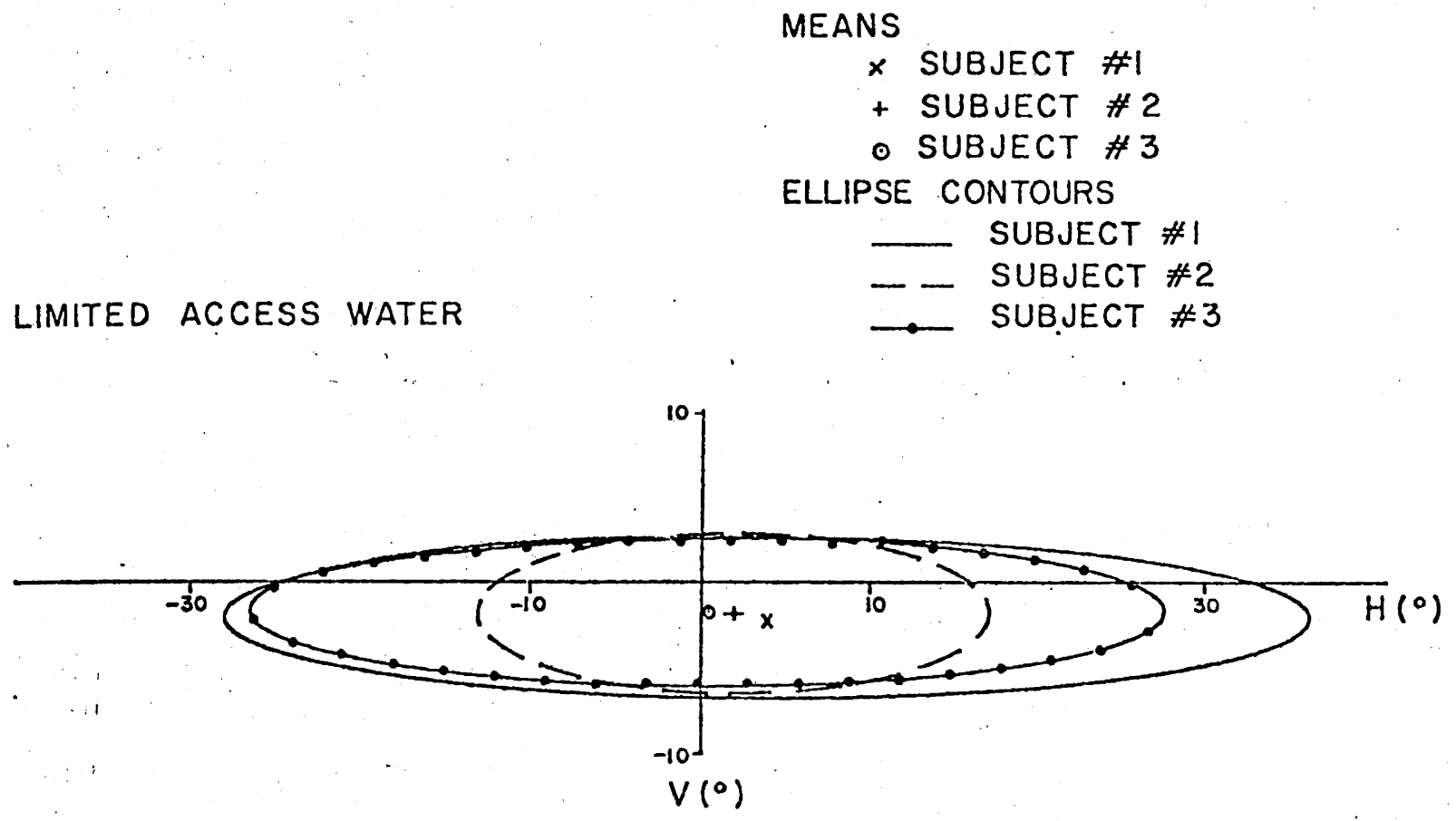

OPEN WATER

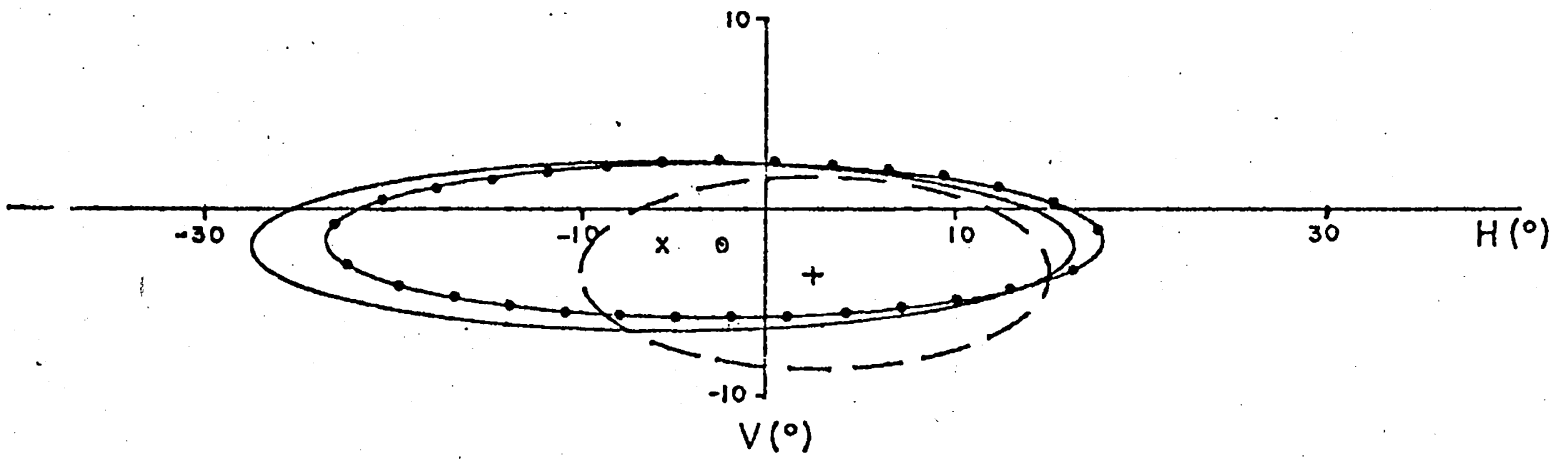

Figure 4.7: Subject-boating environment effects on spatial coordinates 
of straight ahead; however, his pattern to the left of straight ahead, was considerably smaller. The mean horizontal locations were similar for all three subjects in the limited access water condition; while in the open water condition, subjects $\# 1$ and $\# 3$ shifted their mean location to the left of straight ahead and Subject \#2's mean location remained approximately the same.

\section{Components of Variance for Horizontal Location}

Summaries of the resultant data as in Figure 4.8 used the components of varlance determined from the ANOVA results, Although no "subject" main effects were significant for the mean horizontal location, it accounted for almost half the variance of the standard deviation of horizontal location. The main effects due to "task" variables (i.e., velocity, boating environment and navigation task independent variables) were fairly consistent for both the mean and the standard deviation of the horizontal location; however, the "subject-task" components of variance were high (41\%) for the mean horizontal location and negligible. (5\%) for the standard deviation. Furthermore, the error term of the "unexplained" variance was high (35\%) for the mean; whereas it was lower (19\%) for the standard deviation of horizontal Iocation.

The differences related to the boater"s spatial patterns were further explored with an analysis of the magnitude of the distance between fixations as will now be discussed. 

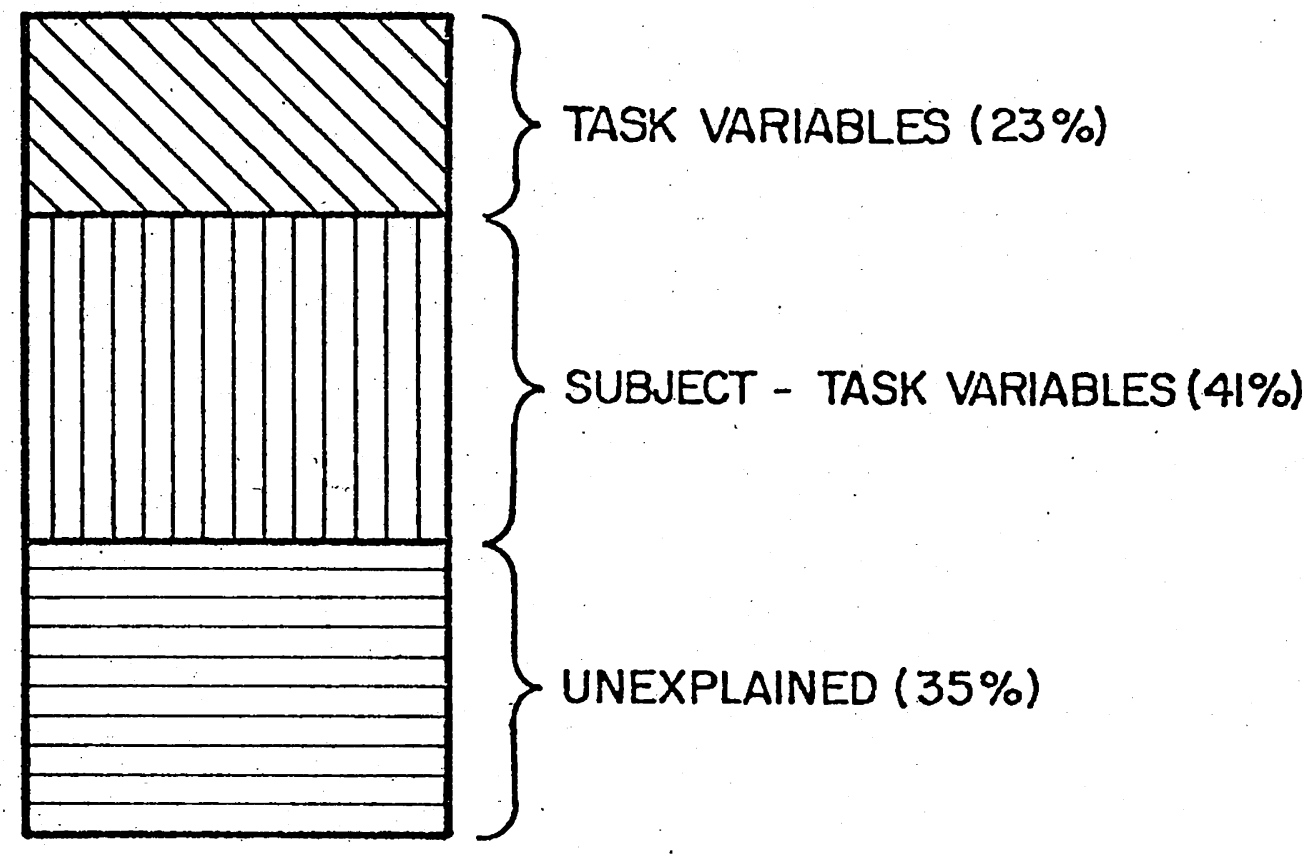

MEAN

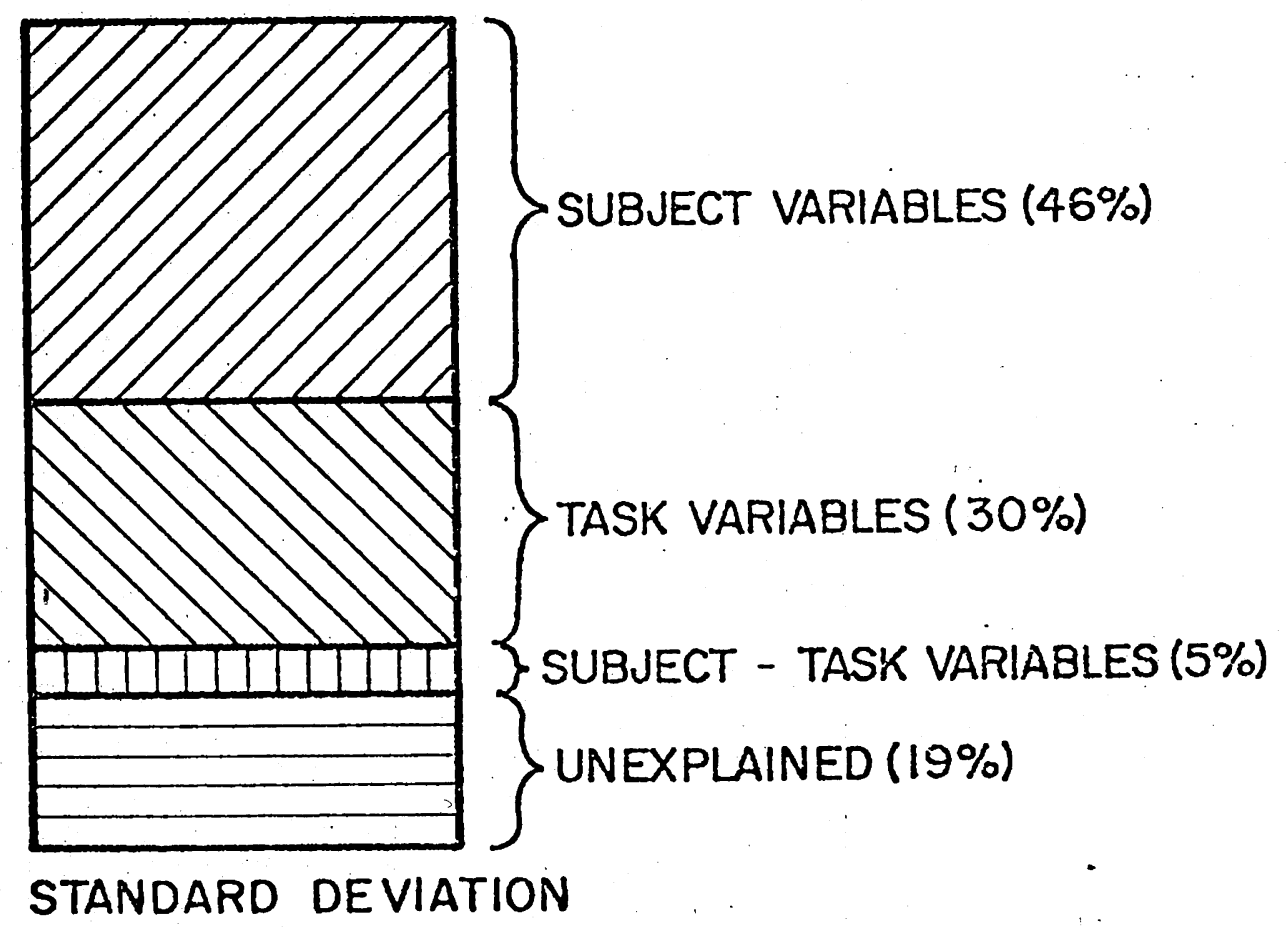




\section{Spatial Patterns - Eye Spot Trave1 Distances}

Eye spot travel distance is another dependent measure which has been used when characterizing operators." spatial scanning patterns. For example, this has been reported for automobile operators and was defined as the distance in degrees between two consecutive fixation locations (Rockwe11, 1971).

In this boating study, extremely large travel distances were caused by certain tasks. As an example, during the compass task, if the boater was fixating straight ahead, say near the horizon, and his next fixation was to the compass, Iong travel distances would obviously occur. Thus, it was decided to delete travel distances to and from navigation targets in order to approximate the "normal" boater's fixation patterns.

Determining the means for these travel distances and then performing an analysis of variance resulted in the significant effects summarized as listed in Table 4.6. These results again amplified some of the effects which have been previously reported for horizontal location.

\section{Subject Effects}

The effect noted in column " $\mathrm{S}$ " of Table 4.6. was caused by Subject \#2. Recall that he had the smallest scanning pattern; he also had the shortest mean travel distances: $\left(9.5^{\circ}\right)$ as compared to Subjects $\# 1$ and \#3 $\left(15.5^{\circ}\right)$. 
Table 4.6

Significant Results from the Analysis of Variance for Eye Spot Travel Distances ${ }^{1}$

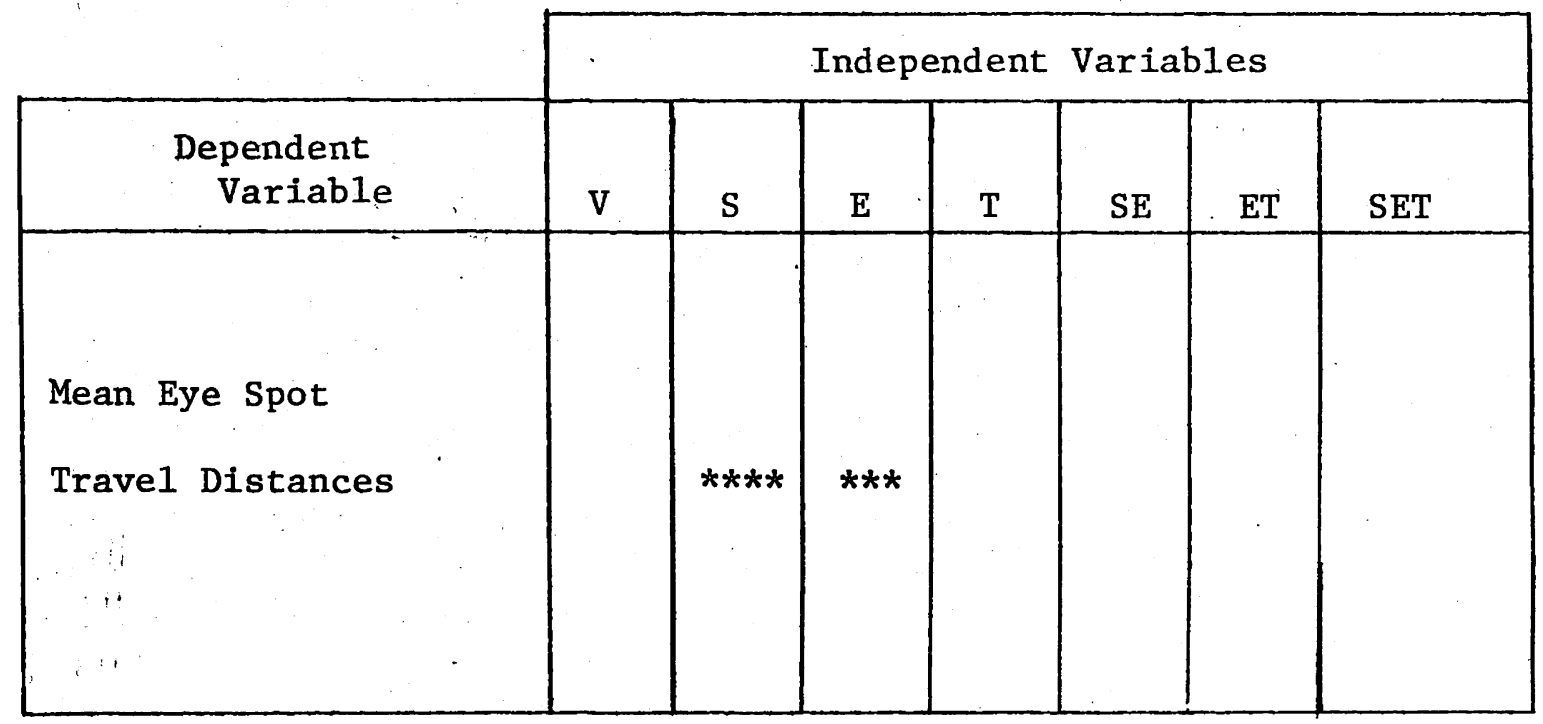

${ }^{1}$ Data sets contain only those fixations not on a particular navigation target or other boats.

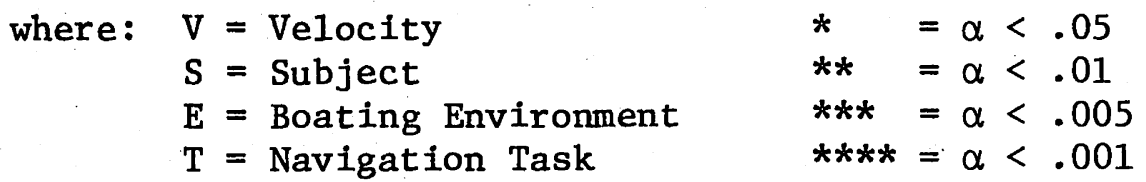

\section{Boating Environment Effects}

The boating environment effect (column E) resulted because the limited access water condition produced larger travel distances $\left(2.5^{\circ}\right.$ greater) than in the open water situation. These results are similar to those found for the standard deviation of the horizontal location. In the limited access water condition, the boaters scanned a larger area and in order to do this they would logically make a larger saccade to a subsequent fixation. 
This concludes the discussion of the parameters specifically related to the boaters' spatial scanning characteristics. The following sections center around the analysis of the temporal eye fixation parameters.

\section{Temporal Characteristics - Fixation Durations}

As previously stated, the navigation types of fixations were removed from the data set. This is important when discussing the durations of fixations to the compass, because at times these fixations were extremely long and tended to have an effect on their respective data sets. Fixations to boats, either moving or anchored, did not seem to effect the duration data set. However, to be consistent, the following discussions exclude all those fixations on either navigation targets or other boats.

Initial analysis of the resultant duration measures revealed that the data were not normally distributed (see example data in Figure 4.9). Most other eye movement researchers apparently either have normally distributed fixation durations or have assumed normal distributions. These durations in the present data were arbitrarily bounded on the bottom end at approximately $50 \mathrm{msec}$, (see Table 4.3, p. 64). Also, the variance (if one were to assume a normal distribution) increased with the mean; thus, a lognormal distribution was a better description of the distribution. Both the Chi-Square and Kolmogorov/Smirnov tests indicated that the lognormal distribution "fit" the duration data (the null hypothesis could not be rejected 


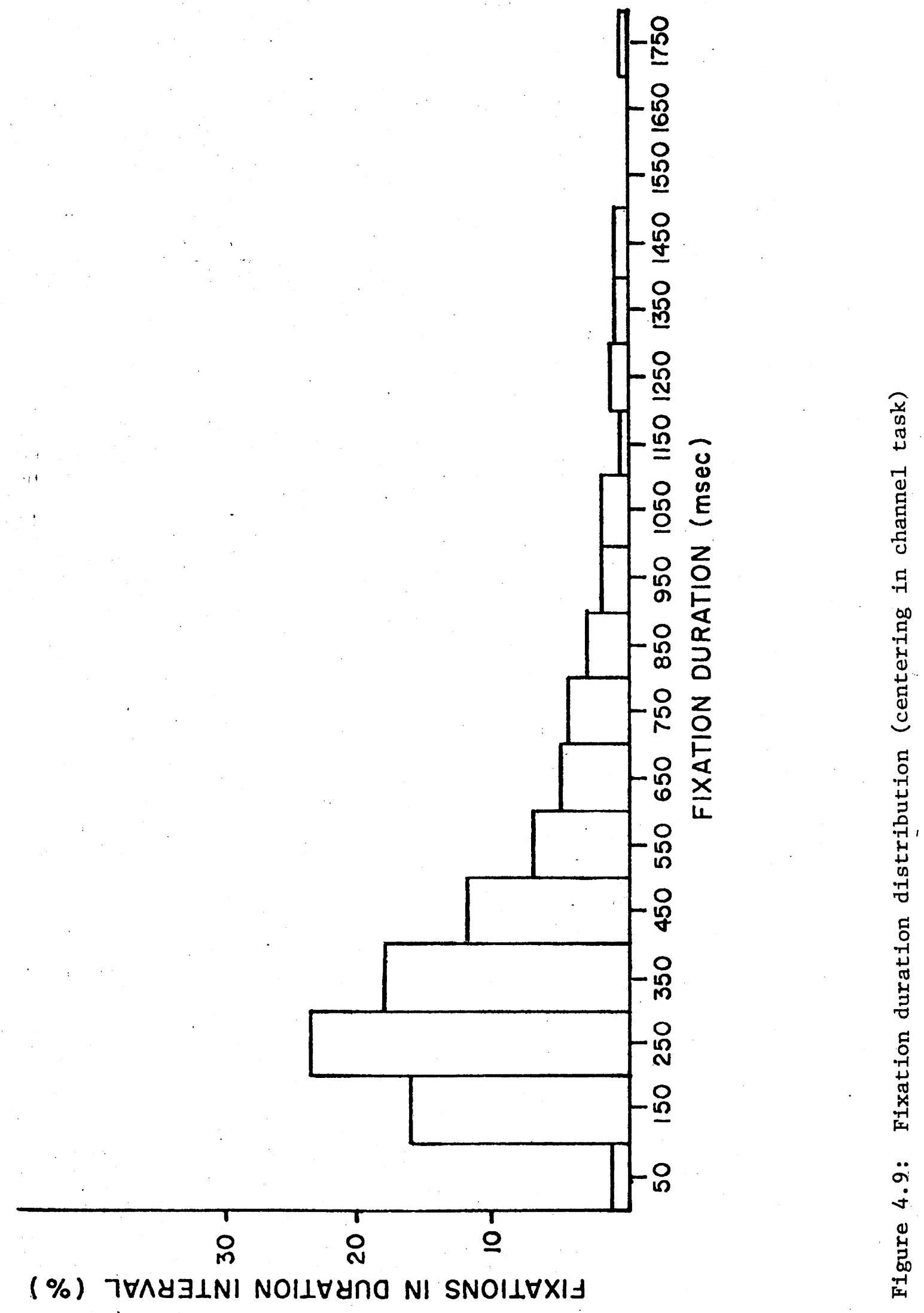


at $\alpha=.05)$. The histogram of the natural logarithmic transformation of the example data in Figure 4.9 is contained in Figure 4.10.

After the $\log$ transformations were obtained for all the duration data, the means were determined. These means were then used in the ANOVA analysis. (For discussion purposes, the untransformed means will be referred to.) Only one significant effect was determined which was due to the velocity-navigation task interaction $(\alpha<.005)$.

This velocity-task effect on fixation, duration is illustrated in Figure 4.11 (using the mean duration values): During the high speed compass and visual reference point tasks, the boaters had significantly shorter mean durations than at the other two velocity levels. However, when the boaters were centering in the channel, the mean durations were shorter during the low velocity than the high velocity tasks.

\section{Fixation Targets}

\section{Analyses with Navigation Targets Excluded}

As previously mentioned, the data reduction also determined the type of object that the subject was viewing for each fixation. For the following analyses, task related fixation, to the compass and other navigation targets were removed. Percent of fixation time per category was then determined. (The 1 ist of all categories of objects is contained in Appendix B.) As seen in Figure 4.12, boaters spent the greatest amount of time fixating on two category types: (1) a general scanning of the water and land and (2) fixations on the instrument pane1. Note that subject 非 consistently exhibited different 


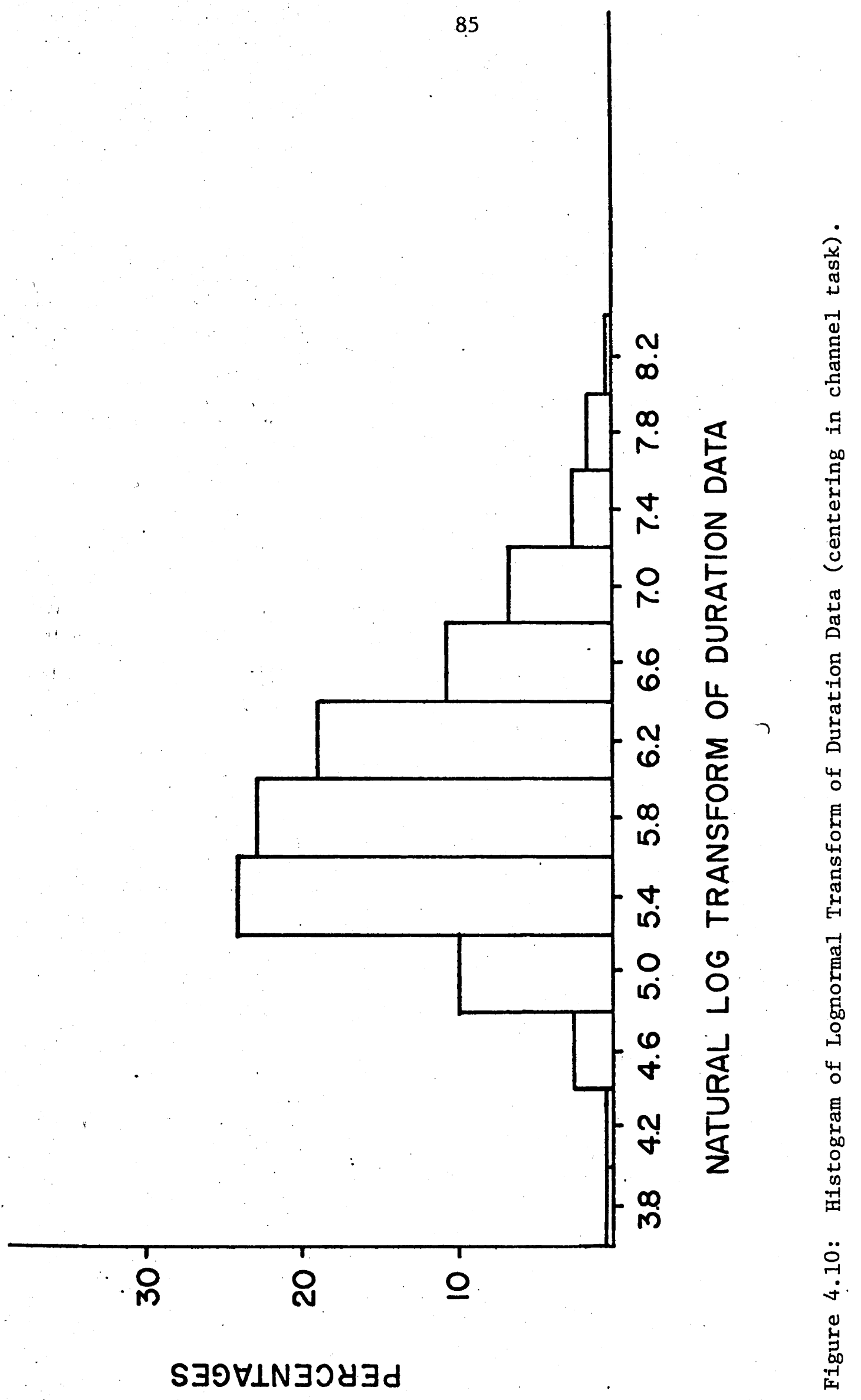


- COMPASS TASK

- - VISUAL REF. PT. TASK

$\rightarrow$ CENTER IN CHANNEL TASK

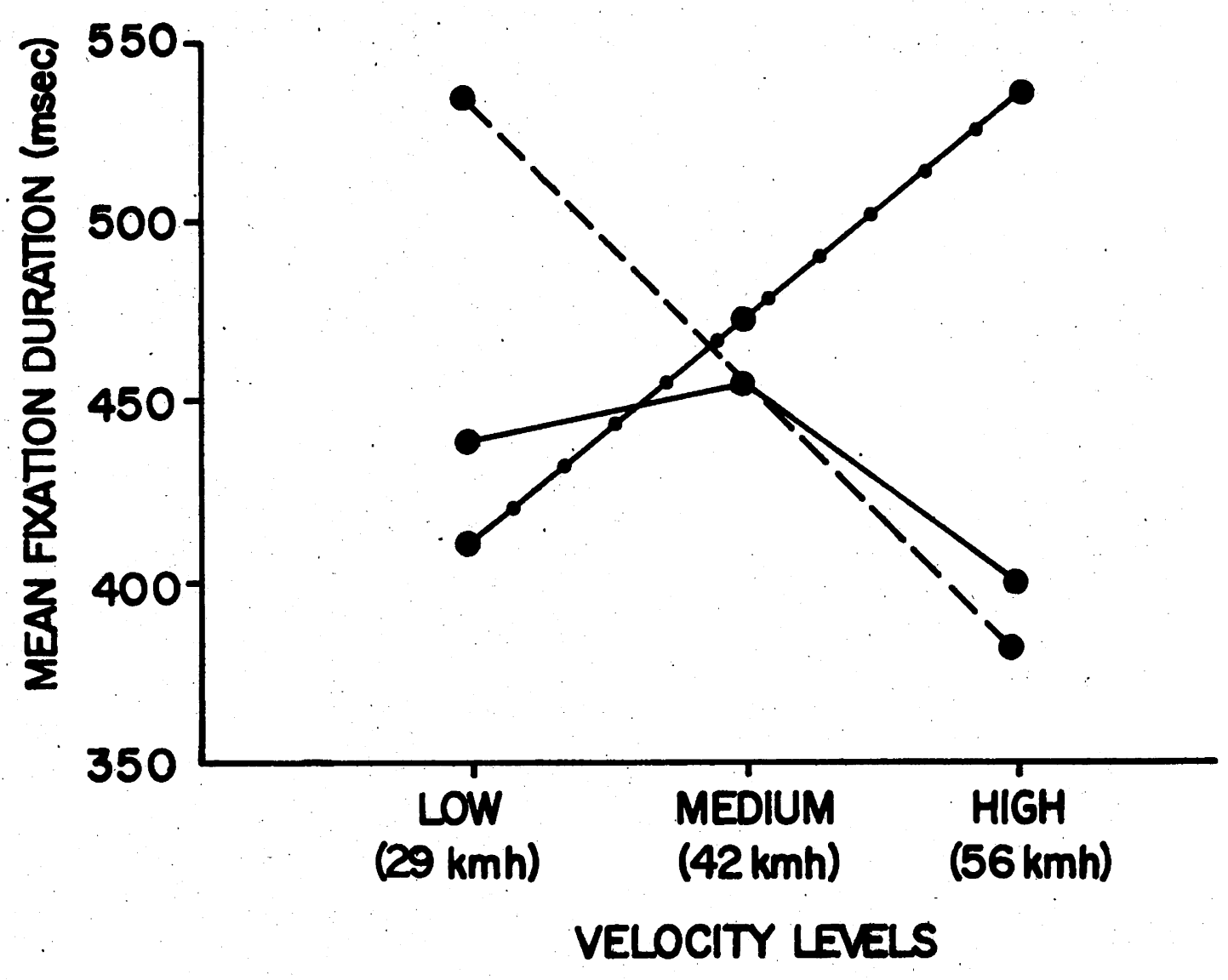

Figure 4.11: Illustration of fixation durations for yelocitynavigation task interaction 

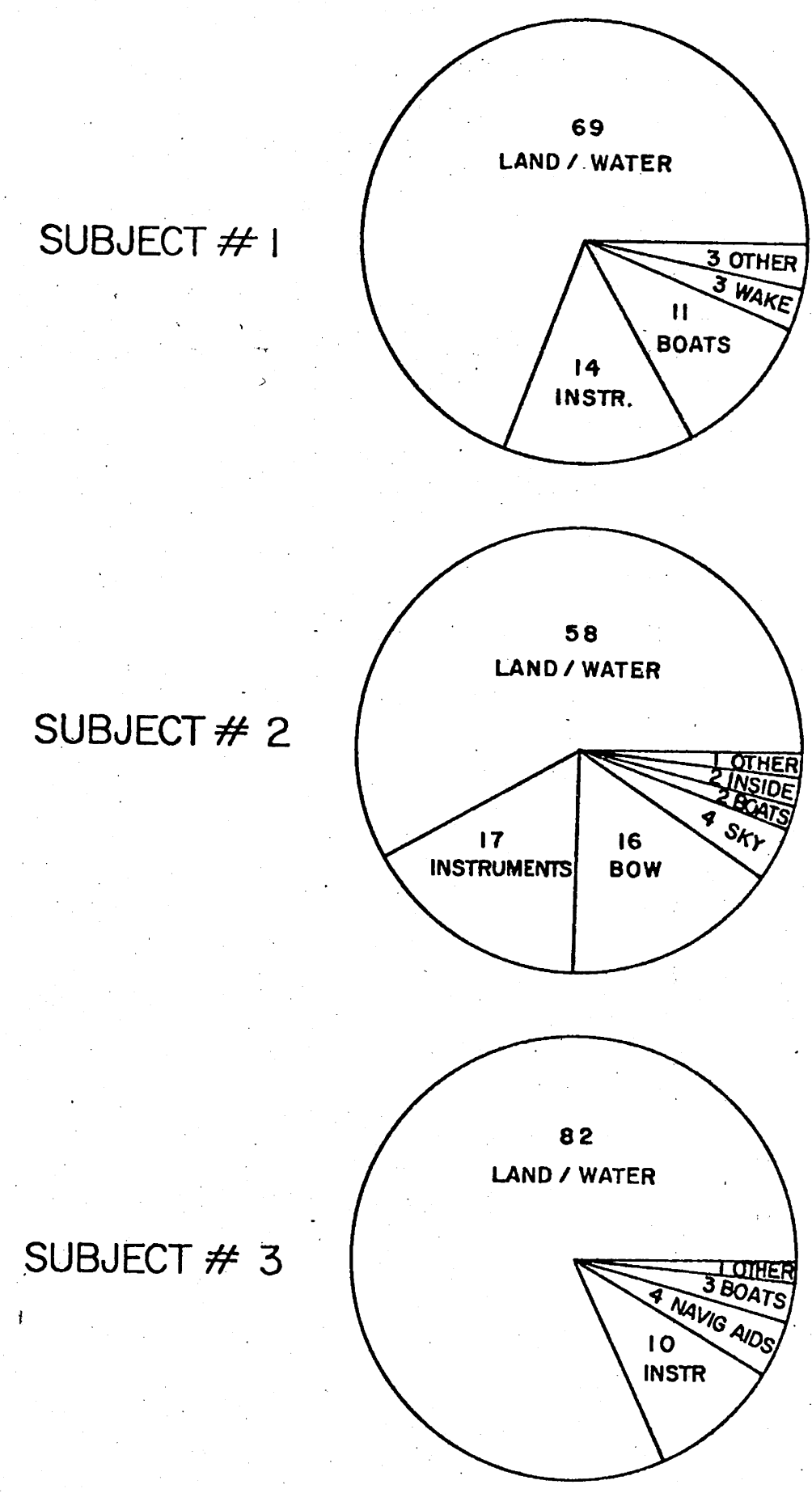

Figure 4.12: Percent fixation time by target type for each subject ${ }^{1}$ ${ }^{1}$ Data sets contain only those fixations not related to the navigation targets 
visual behavior patterns than the other two subjects. Figure 4.12 points out in particular his tendency to look at the bow of the boat, or at. least appear to be doing so. Reasons for this tendency cannot be determined. Similarly, Subject 非 2 had a tendency to look at the sky. These two categories together meant that Subject 非 spent $20 \%$ of his time fixating on irrelevant targets (i.e., the bow of the boat or the sky). However, Subjects $\#_{1} 1$ and $\|_{3}$ did not spend any significant amount of time fixating on these so-called irrelevant targets.

\section{Analysis with Navigation Targets Included}

Most of the previous analyses have deleted fixation targets specific to the navigation task (e.g., compass). It was important to delete these targets since they greatly affected the data sets. However, it is also important to consider the overall effect of these navigation targets on the boaters' visual behavior. Figure 4.13 illustrates the percentage of fixation time the boaters spent on all the different targets including the navigational targets. In this figure, the "visual reference point" and "center in channel" tasks are combined. This was done because these two tasks were not statistically different with respect to fixation time percentages. The compass task was designed to show how fixation patterns would be changed if the driver was preoccupied with something inside his vessel. Figure 4.13 illustrates this change. During the compass task, boaters spent approximately twice as much time (29\%) fixating on it than they spent 


\section{COMPASS TASK}

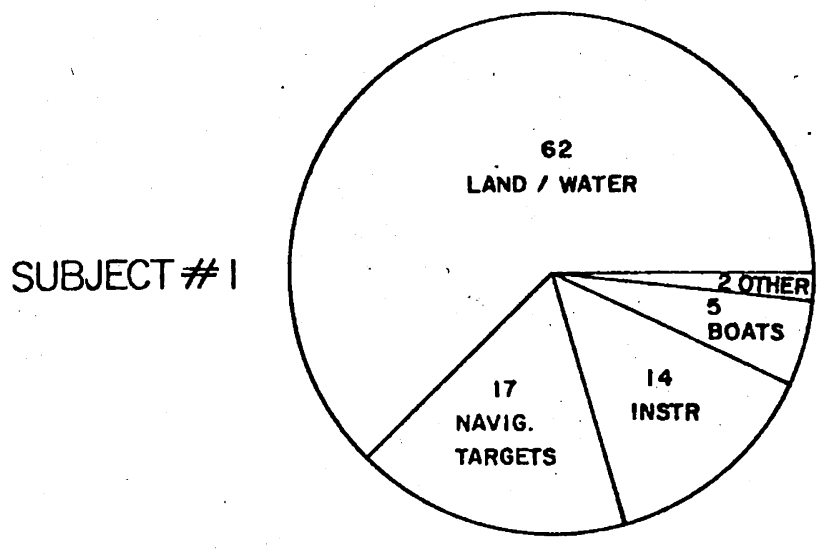

SUBJECT \# 2

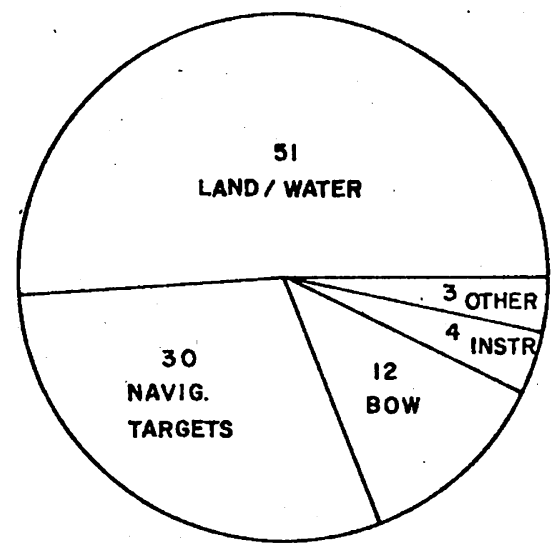

SUBJECT \# 3

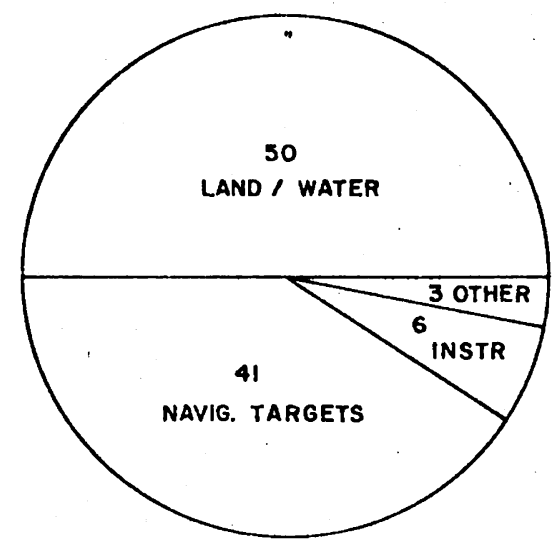

VSUAL REFERENCE POINT AND CENTER IN CHANNEL TASK
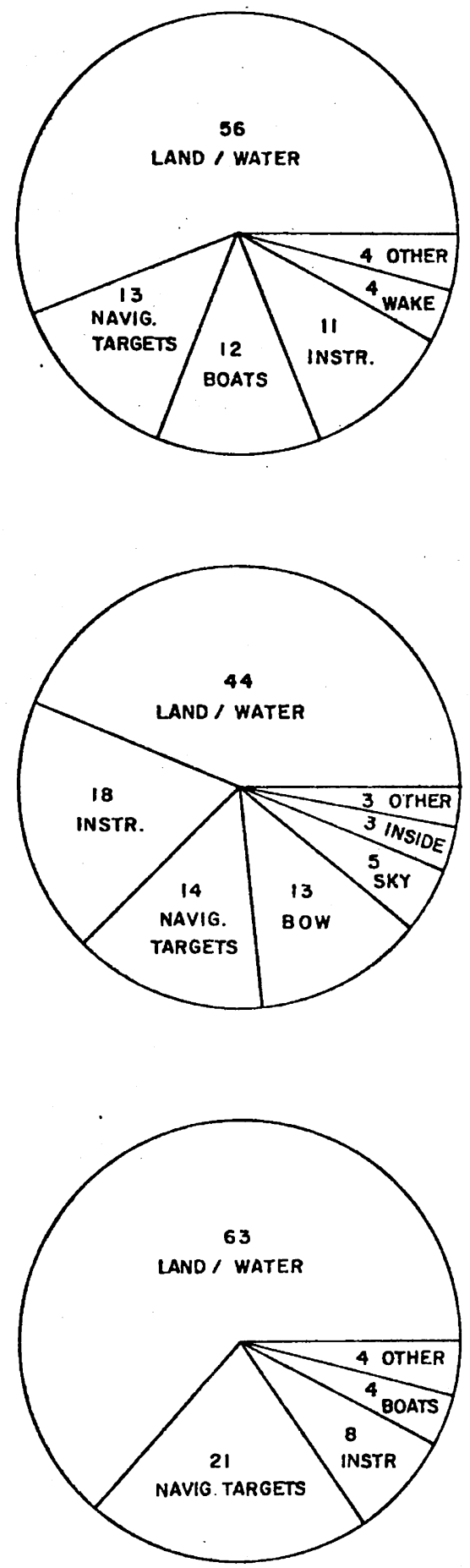

Figure 4.13: Percent fixation time by target type for subjects and navigation tasks ${ }^{1}$

1 Data sets contain all fixations 
fixating on navigation task specific targets for the other two tasks. This meant that less time was spent looking in the vicinity of where there might be potential collision obstacles.

The preceding sections have described and discussed the significant results obtained from the data collected for this research endeavor. Additional analyses utilizing visual zone percentages is contained in Appendix D. These analyses were not included in the main body of this dissertation since it was felt that the results were not as meaningful as those already presented.

Prediction equations of horizontal and vertical fixation parameters were developed in a separate analysis presented in Appendix E. The regression equations developed were capable of predicting the eye fixation parameters (all except one R-Squared was greater than .75). It was of practical importance to find that quadratic effects were significant in many instances; and thus, future research should continue to test many of the variables at a minimum of three levels. The following section compares the boating data to automotive eye fixation data. 


\section{CHAPTER V}

\section{COMPARISON TO AUTOMOTIVE EYE FIXATION RESEARCH}

It was suggested in Chapter I that boat operators' visual fixation patterns might be similar to those of automobile drivers because of a "transfer of training" effect from their own automobile driving experiences. It was this possibility which generated Objective 非 as

a focus of the present research.

In making comparisons of this nature, an initial problem was that of equating speeds on land to speeds on the water. Documented evidence does not exist to equate perceived speed in the two environments. However, this author believes that a $42 \mathrm{kmh}$ water speed can be perceptually equated to an $80 \mathrm{kmh}$ speed on 1and. These speeds appear to be about optimal in that they are: (1) in the medium velocity range for their respective tasks; (2) at a non-stressful perceptual level; and (3) fast enough not to be boring. A particularly good analogy could be drawn between (a) boaters centering their vesse1 in a' channel in a limited access situation and (b) automobile drivers on an open 'highway traveling at the previously mentioned speed levels. Both of these boating and driving environments rely on the operators staying within certain areas which are bounded by the edges of the pathway to the sides of their vessels. The specific comparisons to be discussed are contained in Table 5.1 and Figure 5.1. 
Table 5.1

Comparison of Boat and Automobile Operators' Eye Fixation Data

\begin{tabular}{|c|c|c|}
\hline Dependent Measure & $\begin{array}{l}\text { Boater (Centering } \\
\text { his Vessel in a } \\
\text { Channel) }\end{array}$ & $\begin{array}{l}\text { Automobile } \\
\text { Driver (Open } \\
\text { Highway) }\end{array}$ \\
\hline Mean Horizontal Location & $5^{\circ}$ & $5^{\circ}$ \\
\hline Mean Vertical Location & $-2^{\circ}$ & $2^{\circ}$ \\
\hline $\begin{array}{l}\text { Standard Deviation of } \\
\text { Horizontal Location }\end{array}$ & $22^{\circ}$ & $3^{\circ}$ \\
\hline Mean Eye Spot Travel & $13^{\circ}$ & $2^{\circ}$ \\
\hline Fixation Duration & $540 \mathrm{msec}$ & $270 \mathrm{msec}$ \\
\hline
\end{tabular}

${ }^{1}$ Automobile data derived from Mourant, et a1., 1969.

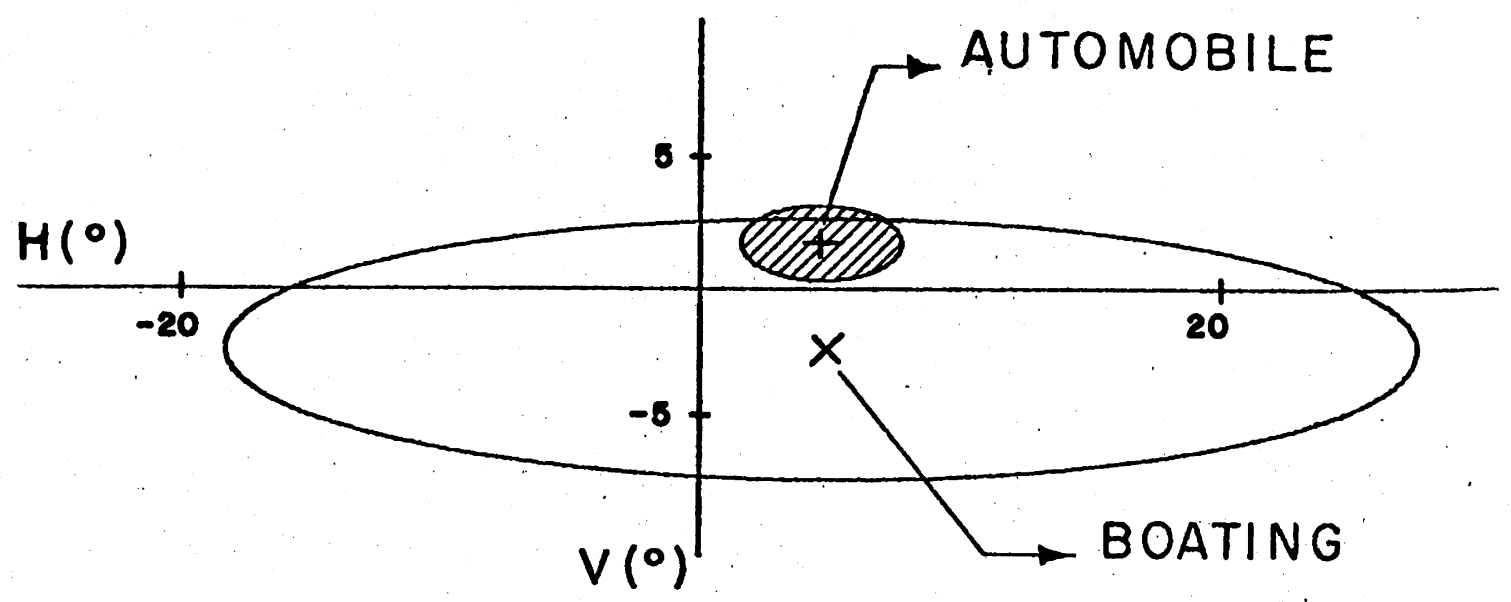

Figure 5.1: Elliptical illustration of automobile drivers' and boaters' spatial scanning patterns (mean \pm 1 standard deviation ellipses)?

${ }^{1}$ Automobile data derived from Mourant, et al., (1969). 


\section{SPATIAL CHARACTERISTICS}

In the centering in channel situation, the boaters' mean fixation location was $5^{\circ}$ horizontal, $-2^{\circ}$ vertical; while Mourant, et al., (1969) found automobile drivers in the open highway situation to have a mean fixation location of $5^{\circ}$ horizontal, $2^{\circ}$ vertical. The mean horizontal locations were remarkably similar, even though the two cockpit stations were on different sides of the vehicles. However, there was a four degree variation in the mean vertical location, which could be related to differences in their primary task. Automobile drivers, concerned with tracking, fixated above the horizon $\left(2^{\circ}\right)$; boaters, concerned with scanning for non-vehicular collision obstacles, fixated below the horizon $\left(-2^{\circ}\right)$.

The standard deviation of the horizontal location was $22^{\circ}$ in boating, compared to the $3^{\circ}$ for automobile drivers found by Mourant, et al., (1969). McDowe11's (1975) analysis of automobile drivers' eye fixation also determined that the horizontal standard deviation was $2-4^{\circ}$.

Another measure of importance when discussing the scanning patterns of these two types of operators, is their eye spot travel distances. Mourant reported a mean travel distance of $2^{\circ}$ for his automobile drivers in the open driving situation. Boaters during their centering in channel tasks displayed a mean travel distance of $13^{\circ}$. These differences in travel distances and standard deviations might be related to the following cognitive processes: 
1. The boaters scanned a larger area to obtain collision avoldance information; thus, larger movements between fixations were necessary. Automobile drivers scanned a smaller area since they were concerned primarily in tracking information.

2. Relevant information for the automobile drivers might have been denser than for the boaters and thus required more foveally related attention fixations.

The spatial analysis of the boaters' eye fixation patterns indicated that their scanning areas were much greater than was found for automobile drivers. Thus, the subjects in this experiment were not apparently seriously affected by a "transfer of training" from automobile driving. If there had been such a "transfer", scan patterns similar in horizontal standard deviations would have been expected. However, it remains an open question as to whether some collisions might be related to a narrow scan pattern, possibly caused by this "transfer".

\section{TEMPORAL CHARACTERISTICS}

Boaters, while centering their yessel in a channel at the medium velocity, had mean fixation durations of $540 \mathrm{msec}$, as compared to a $270 \mathrm{msec}$ mean duration for automobile drivers (see Table 5.1). These differences may be attributed to legitimate task specific differences (such as the amount of visual information to be processed) or possibly to factors in the data recording or reduction techniques. With 
respect to data recording techniques, the earlier work by Mourant was recorded on $16 \mathrm{~mm}$ film which frames approximately four times slower than the boating data collected on video tape. Since one of the criteria for determining a fixation duration was "no eye spot movement" within the included frames, one suspects that the video tape, which frames every $16.7 \mathrm{msec}$, was a better estimator of the beginning and ending of the durations than the $16 \mathrm{~mm}$ film which frames every $62.5 \mathrm{msec}$. This, however, could only explain a part of the difference. Further confusion arises in a more recent study by McPowel1 (1975) wherein a $500 \mathrm{msec}$ mean fixation duration was found for subjects driving an automobile on straight sections of a highway. at speeds of 64 and $96 \mathrm{kmh}$. McDowe11 also used an Ohio State eye movement system similar to Mourant's, except that it used TV cameras rather than $16 \mathrm{~mm}$ film for recordings. One wonders why his fixation duration means were almost double those of the earlier work by Mourant. Another explanation for the longer fixation durations and distances in boating might be related to the amount and type of visual information necessary to be processed. As previously mentioned, the information load may have been heavier and denser in automobile driving than it was in boating. Thus, the automobile drivers may have used shorter durations and shorter distances between fixations in order to input this denser information. The boaters' longer fixations and larger jumps between fixations may have indicated that less dense information over a larger area was being processed; and it is likely 
that peripheral vision may have played a more important role in this process.

McDowe11, in road tests, (1975) also found an increase in fixation durations as velocity increased. These road tests are analogous to this boating study's "centering in channe1" task where a similar effect resulted (increase in fixation durations with increasing veloc1ties; Figure 5.2). Unfortunately, McDowell's automobile drivers were only tested at two speed levels and thus, any quadratic effects which existed could not be determined. However, even from McDowell's limited number of velocity levels, it is apparent that his data can not be equated with boaters who performed the other boating tasks.

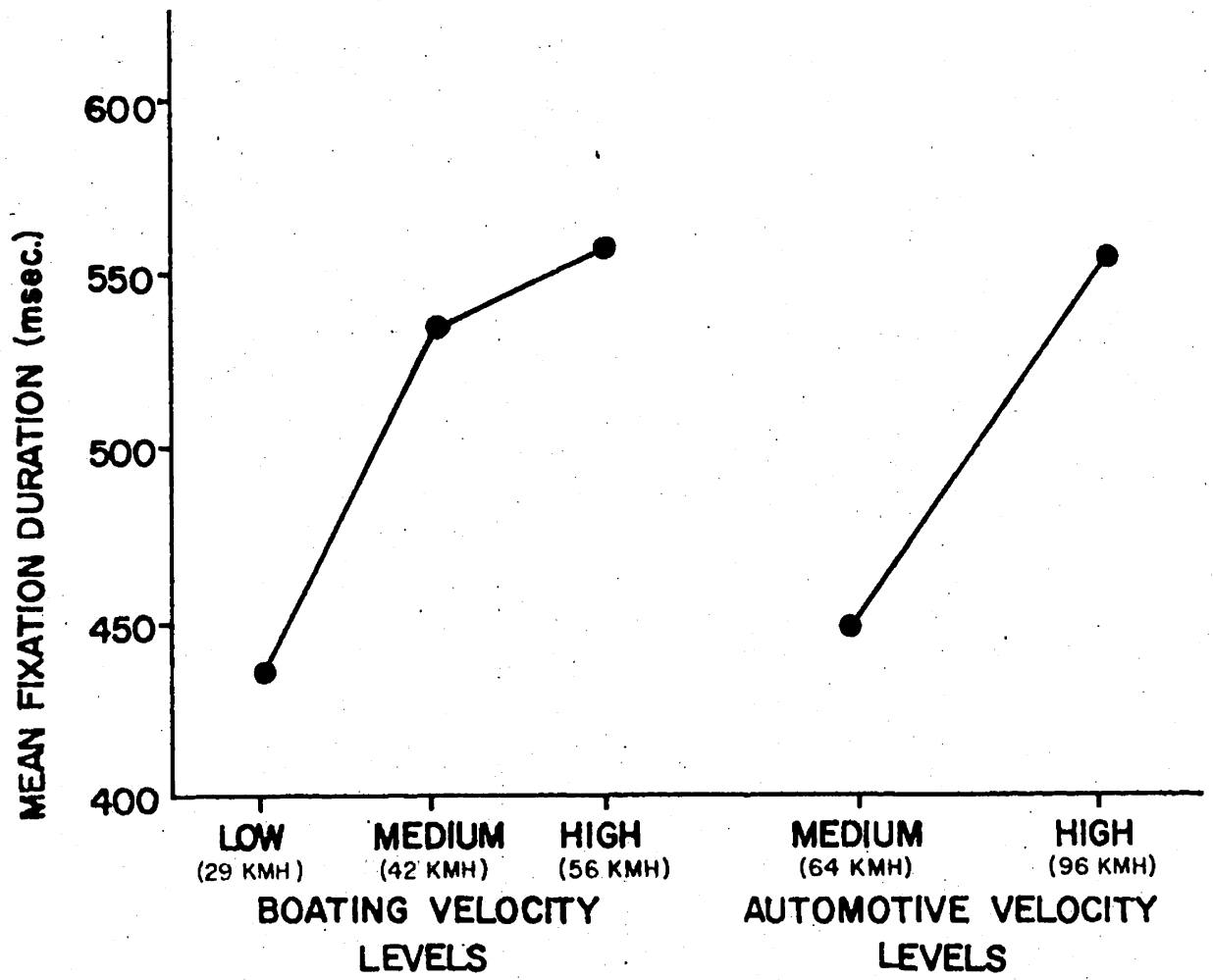

Figure 5.2: Velocity effects: boating center in channel task vs. McDowe11 (1975) automobile driving 
During these other two tasks, (the compass or visual reference point), boaters exhibited decreases in the duration parameters as the velocity increased. Several basic types of visual behavior are emerging from these above boating data.

A first type of basic behavior might have been exhibited during the centering in channel task and in McDowell's (1975) road task. He explained his increases in mean fixation durations with increased velocity as being attributable to drivers making more accurate discriminations of their visual information. For boaters centering their vessel in extremely deep channels, the emphasis on collision avoidance may have been reduced since the probability of non-vehicular collision objects was lower. Thus, in this task situation, boaters may have been primarily concerned with tracking or lateral placement; and at higher yelocities this lateral placement discrimination may have required more accurate information processing of peripheral information, in particular.

A second basic type of visual behavior may have been exhibited during the compass and the visual reference point tasks (Figure 4.11) where collision avoidance was a primary concern. In these particular task situations, the routes and water depths were variable and the probability of encountering a non-vehicular collision object was higher; and at the higher velocities, boaters made more fixations of shorter durations. This may have been a strategy to briefly sample a location and make a yes/no response with respect to such things as debris in the water. 
A third type of behavior relates to the observation of instruments which supply quantitative information. Mourant and Rockwel1 (1972) found that experienced automobile drivers looking at a speedometer had mean glance durations of approximately $780 \mathrm{msec}$. The boaters had two speed monitoring devices, the speedometer and the tachometer; and these boating subjects were instructed to maintain certain tachometer settings. The glance duration for the boaters viewing either of these instruments was $930 \mathrm{msec}$. Thus, the durations necessary for obtaining quantitative information from instruments is conslderably longer than durations related to qualitative information from the forward visual field outside of the cockpit area.

\section{SUMMARY OF COMPARISON CHARACTERISTICS}

The comparison of boaters to automobile drivers made in this Chapter $\mathrm{V}$ to address Thesis Objective \#3 has, in summary, provided the following insights:

\section{Spatial}

While centering in a channe1, boaters" mean horizontal fixation locations were similar to automobile drivers, although the standard deviations were considerably greater. Thus, boaters' fixations were distributed over a larger portion of the forward viston field (see FIgure 5.1).

The mean vertical fixation locations indicated that boaters. scanned below the horizon, in contrast to automobile drivers who 
scanned above the horizon (see Figure 5.1). This may have been related to a difference in their primary tasks; boaters were interested in collision avoidance and automobile drivers with tracking and lateral placement.

\section{Tempora1}

A significant velocity-task interaction was found for the fixation duration measure (Figure 4.11). It was suggested that three basic types of visual search behavior might have been displayed.

A first was exhibited during the centering in channel task, where boaters were similar to automobile drivers in that their fixation durations were longer as velocity increased (Figure 5.2). This may mean that more accurate discriminations of the visual information related to possibly the lateral tracking task were being made.

A second behavior was displayed during the compass and visual reference point tasks, where boaters had shorter durations at increased velocity. This may indicate that boaters were sampling visual information at a faster rate in order to make a series of binary yes/no responses concerning potential collision obstacles.

And finally, a third behavior occurred where quantitative information was being observed from the speedometer or tachometer instruments. Here, fixation durations were longer than for any fixations occurring for out of cockpit tasks. 


\section{CHAPTER VI}

\section{SUMMARY AND RECOMMENDATIONS}

The following summarizes the findings from this research and discusses these findings in relationship to recommendations for future boating research.

\section{OBJECTIVE 非: COLLECTION OF BOATER EYE FIXATION DATA}

The first research objective involved (a) determining the feasibility of collecting boaters' eye fixation data and (b) establishing a research methodology for this type of data collection.

With respect to the feasibility of collecting accurate boater eye fixation data, the eye spot calibration error during testing was, at the most, $6 \%$ of the standard deviation of the horizontal fixation location; this calibration error was known for each run and could be corrected before the raw data was summarized and analyzed.

Chapter III described in detail the methodology: utilized. This included the selection of the independent variables; the determination of subject sample size statistical criterion, and the discussion of details concerning test procedures.

Chapters II and III presented evidence of the development of a methodology to collect boater eye fixation data and, thus, satisfy Objective \#1. Sufficient details were given within these chapters to allow future boating researchers to conduct their own studies in ways which will hopefully confirm and supplement the findings presented herein. 
OBJECTIVE 非: SOME FACTORS AFFECTING BOATERS' VISUAL BEHAVIOR

Objective 非 2 was related to determining some factors which affected boaters' eye fixation behavior. Chapter IV contained the analyses of these factors and determined those which did significantly affect the dependent measures. The following sections summarize these factor effects.

\section{Boating Environment Navigation Task Effects}

Chapter IV included discussions concerning the effects of the boating environment factor (open water vs. 1imited access water) and the navigation task factor (compass vs: center in channel vs. visual reference point tasks).

\section{Fixation Locations}

With respect to the boating environment, the limited access water condition was responsible for boaters scanning a signiffcantly larger area to the right of the vessel than the open water condition (Figure 4.5, pg. 75). More fixations to the right during this condition might be related to the cockpit station being located on the right (stárboard) side of the boat.

The navigation tasks generated statistically different distributions of horizontal fixations (Figure 4.4, pg. 73). Boaters scanned the largest area during the visual reference point task. Since this reference point task is probably the most common boating task, it is 
reassuring to know that the scanning patterns were large. In the compass task the boaters' fixation locations were centered at $-3^{\circ}$ horizontal, wherein the channel task produced a mean horizontal location of $+3^{\circ}$. Again, a preference for looking to the right side of the vessel during this channel task may be related to boaters' favoring the lateral position cues closest to their cockpit station.

\section{Fixation Durations}

Duration parameters were found to be significantly different due to a velocity-navigation task interaction (Figure 4.11, pg. 86). During the channel tasks, the durations increased with increased velocities. However, during the compass and reference point tasks, these durations decreased with increased velocities.

Snyder (1973) suggested that fixation duration could be used as an inverse indicator of visual acquisition performance, and Loftus (1976) found memory performance to be related to number of fixations per target. Utilizing these results one infers that the boaters may be approaching a more efficient visual performance during the high speed compass and visual reference point tasks.

The duration results further suggest that a speed/accuracy tradeoff might have been displayed by these boaters. During the channel task the durations increased with increased velocities. As previously mentioned, McDowe11.(1975) related similar increases in automobile drivers' durations to the processing of information more accurately. 
The boaters exhibited a decrease in durations during the other two tasks, which may be related to an increased information processing rate. This velocity-task interaction should be further studied in order to determine the nature of the boaters' various search strategies.

\section{Subject Effects}

Subject $\# 2$ displayed visual behavior which was statistically different from the other subjects (see Figure 4.7, pg. 77). These differences were primarily seen in his much smaller spatial scanning patterns. When such extreme differences are found for one subject, one is tempted to remove this subject's data because of possible unknown factors in the data or methodology. However, this idea was rejected because no such factor could be identified; and subject 非, although not similar to the other subjects, may still represent a portion of the boating population who have legitimate smaller scanning patterns.

It is possible that Subject $\#_{2}$ did not realize the importance of visually scanning a large area for collision avoidance monitoring. In addition to smaller scanning patterns Subject $\left.\right|^{2}$ spent $20 \%$ of his time fixating on objects which were apparently irrelevant to his boating task (Figure 4.12, p. 87). This might mean that he was easily distracted from his primary task. 
OBJECTIVE \#3: COMPARISON OF BOATER VERSUS AUTOMOTIVE VISUAL BEHAVIOR PATTERNS

The data related to Objective 3 were presented in Chapter V. This comparison of the boating and automotive eye fixation data illustrated some differences between the two operators (see Figure 5.1, p. 92). While centering in a channel, boaters' mean horizontal locations were similar to automobile drivers in that both were about $5^{\circ}$ right of center. However, the standard deviation of $22^{\circ}$ for boaters was much larger than the $3^{\circ}$ found for automobile drivers (see Table 5.1, pg. 92). Mean vertical locations indicated that boaters scanned below the horizon $\left(-2^{\circ}\right)$, while automobile drivers scanned above the horizon $\left(2^{\circ}\right)$.

\section{FUTURE RESEARCH RECOMMENDATIONS}

This section is divided into two parts. The first is related to those research recommendations developed from the literature review. The second part concerns recommendations which were an outcome of the data analyses and discussions.

\section{Recommendations Related to Literature Review}

The boating research by MacNeill, et al., (1976a) had subjects both maintain a compass heading and also monitor the VAST light task. However, judgments were never made as to the degree of attention time devoted to elther of the tasks. Utilizing an eye fixation approach, 
one could determine the respective attention time for each task. This type of information might also be useful in determining the "stress" levels that subjects were experiencing. Such "stress" levels could be increased by difficult compass headings, boat traffic or water conditions. Changes in a subject's peripheral light detection capability (VAST test) might be demonstrated further as being a function of stress levels. A subject's percent fixation time on a given task or his durations of fixations might be used as measures of such stress.

As previously stated, collision avoidance is an important task for boaters. These boaters should be constantly monitoring for either vehicular or non-vehicular objects. Future boating researchers should address the issue of vehicular collision avoidance. A possible method for this type of research would be to monitor boaters' eye fixations on another vessel as a function of such items as the other boat's distance, angle of approach, and velocity. The non-vehicular collision aspect should also be further investigated by studying a boater's eye fixation to these non-vehicular objects as a function of object type, contrast level and the boater's own stress level.

\section{Recommendations Related to Resultant Boating Data}

The analyses of the resultant data revealed several significant factors which affected eye fixation behavior. The scanning of a larger area to the right during the limited access water situation 
was suggested as possibly being related to their cockpit station 10cation. Examining the eye fixation patterns of boaters operating center or left helm station vessels would be useful. The importance of such research lies in determining whether boaters are "favorịng" a certain side of their vessel and whether the "unfavored" side would have a higher collision potential.

The navigation task-velocity effects on the duration parameters could be further researched using a secondary task approach. The changes in fixation durations as a function of velocity might be related to spare information processing or even speed/accuracy trade-offs.

Many dissimilarities were noted between. boat and automobile drivers' eye fixation data. These data were admittedly collected on different subjects utilizing different equipment and data reduction/analyses techniques. To alleviate discrepancies due to these items, an eye fixation study could be conducted using the same subjects driving a boat and an automobile. Subjects could be asked to perform analogous tasks in both vehicles. The boat could also be equipped with a comparable automotive type mirror system to determine its effects on boaters' fixation patterns.

It can be seen from this chapter that a considerable amount of information has been gained about power boat operators" visual behavior patterns. Some of these may be quite useful to those interested in collision related behavior. The examples of proposed research suggest that there are other interesting and useful endeavors to be undertaken in this new application of human performance. 


\section{BIBLIOGRAPHY}

Anliker, J. "Eye Movments: On-Line Measurement, Analysis, and Control." Eye Movements and Psychological Processes. Edited by R.A. Monty and J.W. Senders. Hillsdale, N.J.: Lawrence Erlbaum, Assoc., 1976.

Barnoski, R.L.; Maurer, J.R. and Kugler, B.A. Assessment of Automotive Visibility by a Figure of Merit. New York: Society of Automotive Engineers Report No. 700394, 1970.

Bartz, A.E. Mechanisms of Eye and Head Movements in Driving Tasks, Moorhead, Minn.: Concordia College, 1965.

Bewley, L.A. "Spectacle Frames Reduce the Field of Vision: A Driving Hazard." Journal of American Optometrists Association, June, 1969, 64-69.

Bhise, V.D. "Visual Search by Drivers in Freeway Merging: Implications for Vehicle Design." Proceedings of the 17th Annual Meeting

of the Human Factors Society, Washington, D.C., October 1973,

Bhise, V.D. and Rockwe11, T.H. "The Role of Peripheral Vision and Time Sharing in Driving." Proceedings of the 15th Conference of the American Association for Automotive Medicine. Colorado Springs, Colorado, October, 1971, 320-341.

Burg, A. "Lateral Visual Field as Related to Age and Sex." Journal of Applied Psychology, 52 (1968a), 10-15.

Burg, A. The Relation Between Vision Quality and Driving Record. Presented at the Highway Research Board Symposium on Visibility in the Driving Task, Texas A \& M University, May, 1968b.

Carpenter, P.A. and Just, M.A, "Linguistic Influences on Picture Scanning." Eye Movements and Psychological Processes, Edited by R.A. Monty and J.W. Senders. Hillsdale, N,J.: Lawrence Erḷbaum, Assoc., 1976.

Devlin, W.A. and Roe, R.W. The Eyellipse and Considerations in the Drivers Forward Field of View. New York: Society of Automotive Engineers, Report Na. 680105, 1968.

Dixon, W.J., ed. BMD - Biomedical Computer Programs. Los Angeles: University of California Press, 1974.

Domey, R.G. and McFarland, R.A. "Dark Adaptation Threshold, Rate, and Individual Prediction." Highway Research Board Record, 298 (1961), 3-17. 
Dykstra, D.R. Effect of Head-Mounted Visual Monitoring Equipment on Operatar Behavior, Unpublished Masters Thesis, the University of Michigan, 1977.

Fitts, P.M.; Jones, R.E. and Milton, J.L. Eye Fixations of Aircraft Pilots, III. Frequency, Duration, and Sequence Fixations When Flying Air Force Ground-Controlled Approach System (GCA). USAF Technical Report No, 5967; Wright-Patterson Air Force Base, February, 1950.

Ford Motor Co: Field of View from Automobile Vehicles. Dearborn, Michigan: Automotive Safety Planning and Research Office, Report No. S-72-6, September, 1972.

Fox, D.J. and Guire, K.E. Documentation for MLDAS, Michigan Interactive Data Analysis System. Ann Arbor, Michigan: Statistical Research Laboratory of The University of Michigan, 1973.

Fry, C.A. Visual Performance Under Varying States of Adaptation. Presented at the Highway Research Board Symposium on Visibility in the Driving Task, Texas A \& M University, May, 1968.

Gould, J.D. "Looking at Pictures." Eye Movements and Psychological Processes. Edited by R.A. Monty and J.W. Senders. Hillsdale, N.J.: Lawrence Erlbaum, Assoc., 1976.

Hahn, G.J. and Shapiro, S.S. Statistical Models in Engineering. New York: John Wiley and Sons, Inc., 1967.

Hicks, C.R. Fundamental Concepts in the Design of Experiments. New York: Ho1t, Rinehart and Winston, 1973.

Jones, R.E.; Milton, J.L. and Fitts, P.M, Eye Fixations of Afrcraft Pilots, I. A Review of Prior Eye-Movement Studies and A Description of a Technique for Recording the Frequency, Duration, and Sequences of Eye Fixations During Instrument Flight, USAF Technical Report No. 5837, Wright-Patterson Air Force Base, September, 1949.

Kaluger, N.A. and Smith, G.L. "Driver Eye-Movement Patterns Under Conditions of Prolonged Driving and Sleep Deprivation." Highway Research Record, 336 (197.0), 92-106.

Lambert, R.H.; Monty, R.A, and Hall, R.J, "Methods and Designs-High-Speed Data Processing and Unobtrusive Monitoring of Eye Movements." Behavior Research Methods and Instrumentation, $6(1974), 525-530$. 
Loftus, G.R. "A Framework for a Theory of Picture Recognition." Eye Movements and Psychological Processes. Edited by R,A. Monty and J.W. Senders. Hillsdale, N.J.: Lawrence Erlbaum Assoc., 1976.

MàcNe111, R.; Stieh1, C.; Cohen, S. and Do11, T. Recreational Boat Safety Collision Research - Phase II, Vol. I - Problems Definition: Safety Enhancement Concepts. Final Report for the U.S. Coast Guard by Wyle Laboratories, 19.76a.

MacNeil1, R. and Cohen, S. Recreational Boat Study Collision Research - Phase II, Vol. II - Collision Accident Investigations 1975. Final Report for the U.S. Coast Guard by Wyle Laboratories, $\overline{1976 b}$.

MacNei11, R.; Momany, N.; Stieh1, C. and Lancaster, G. Recreationa1 Boat Safety Collision Research. Phase I, Vol. I - Problems Definition. Final Report for the U.S. Coast Guard by Wyle Laboratories, NTIS No. AD A015 819, 1975.

Mansour, T.M. Driver Evaluation Study of Rear View Mirror Reflectance Levels. Presented at the Mid-Year Meeting of the Society of Automotive Engineers, Montrea1, Canada, June, 19.71.

Marcus, K.H. Periscopic Rear Vision in Automobiles. Presented at the Mid Year Meeting of the Society of Automotive Engineers, Detroit, Michigan, May, 1968.

McDowe11, E.D. An Exploratory Investigation of the Stochastic Nature of the Driver's Eye and Control Movements and Their Relationship to the Roadway Geometry. Unpublished doctoral thesis, Ohio State University, 1975 .

Miller, J.M.; Gatche11, S.M. and Dykstra, D.R. The Visual Behavior of Recreational Boat Operators. Report for the U.S. Coast Guard by the University of Michigan, 1977.

Miller, J.M. H Human Factors Applications in Boating Safety: Final Report for the U.S. Coast Guard by the University of Michigan, NTIS No. AD-781-205, 1973.

Moskowitz, H.; Ziedman, K. and Sharma, S. "Visual Search Behavior While Viewing Driving Scenes Under the Influence of Alcohol and Marijuana," Human Factors, 18 (October, 1976), 417-432,

Motor Vehicle Safety Standard No. 107: Reflecting Surfaces - Passenger Cars, Multipurpose Passenger Vehicles, Trucks, and Buses. (Effective: January 1, 1968), Washington, D.C.: The Bureau of National Affairs, Inc., 1973. 
Mourant, R.R. and Donahue, R.J. Mirror Sampling Characteristics of Drivers. Warrendale, Pa,: Society of Automotive Engineers, Report No. 740964,1974 .

Mourant, R.R. and Rockwe11, T.H. "Strategies of Visual Search by Novice and Experienced Drivers," Human Factors, 14 (1972), 325-335.

Mourant, R.R.; Rockwe11, T.H. and Rackoff, N.J. Drivers" Eye Movement and Visual Workload. Presented at the 48th Annual Meeting of the Highway Research Board, Washington, D.C., January, 1969.

Newsome, L.R. A Perceptual Factor that Could Contribute to Road Accidents, Crowthorne, England: Road Research Laboratory, RRL Report LR 135, 1967.

Pettit, G.D. Human Factors Analysis of Rear View Mirrors for Motor Vehicles. Aberdeen Proving Grounds, Maryland, Human Engineering Lab, AMCMS Code 4510.14,3500,2, October, 1966,

Rockwe11, T.H. and Weir, F.W. "The Effects of Carbon Monoxide Intoxication on Human Performance in Laboratory and Driving Tasks." Proceedings of the First International Conference on Driver Behavior, Zurich, Switzerland, 1973.

Rockwe11, T.H.; Bhise, V.D. and Mourant, R.R. A Television System to Record Eye Movements of Automobile Drivers. Presented at the Society of Photo-Optical Instrumentation Engineers, Dearborn, Michigan, November, 1972.

Rockwe11, T.H. "Visual Acquisition of Information In Driving Through Eye-Movement Techniques: An Overview." North Carolina Symposium on Highway Safety, (1971), 67-95,

Rockwe11, T.H.; Ernst, R.L. and Rulon, M.J. Visual Requirements in Night Driving. Washington, D.C,: Highway Research Board, 1970,

Rockwe11, R.H.; Overby, C. and Mourant, R,R. "Driver Eye Movements: An Apparatus and Calibration." Highway Research Record, 247 (1968), 29-38,

Rutley, K.S. and Mace, D,G. A Preliminary Investigation into the Frequency of Driver Motor Actions and Eye Movements. Crowthorne, Berkshire, England: Road Research Laboratory, 1968.

Salvatore, Santo. "The Estimation of Vehicular Velocity as a Function of Visual Stimulation." Human Factors, 10'(1968), 27-32. 
Senders, J.W., Kristofferson, A.B., Levison, W.H.: Dietrich, C,W. and Ward, J.L. Attentional Demand of Automobile Driving. Cambridge, Mass: Bolt, Beranek and Newman, Inc., Report 1482, March, 1967.

Senders, J.W. "A Re-Analysis of the Pllot Eye-Movement Data." IEEE Transactions on Human Factors in Electronics, 7 (June, $1966), 103-106$.

Shinar, D.; McDowe11, E.D. and Rockwe11, T.H. "Eye Movements in Curve Negotiation." Human Factors, 19 (February, 1977), 63-71.

Smith, 盀.P., and Weale, R.A. "Obstruction of Vehicle-Drivers" Vision by Spectacle Frames." British Medical Jounnal, 2 $(1966), 445-447$.

Sinyder, H.L. "Dynamic Visual Search Patterns." Visual Search, Washimgtion. D..C.: National Academy of Sciences, 1973,

Sowa, W.M. and Fraser, T.M. MSystems Safety in Recreationa1 and "Powered Velhicles." Human Factors, 16 (October, 1974), 474-480.

Stieimman, R.M. '"Role of Eye Movements in Maintaining a Phenomenally Cliear and "Stable World." Eye Movements and Psychological Processes. Edited by R.A. Monty and J.W. Senders. Hillsdale, N..J.o: Lawrence Erlbaum, Assoc., 1976.

Stifehi, (C. A Telephone Survery to Determine the Effects of Navigation Lights on Nightime Collisions. Huntsville, Alabama: Wylle Laboratories, Contract No.e DQT-CG-40, 672-A, May 1975.

U..S. (Coast Guard. Boating Statistics 197.2, CG-357. Washington, D..C.a: Department of Transpontation. 1973.

Whallen, J..T.: Rockwel1, T..H., and Mourant, R.R. A Pilot Study of Dritivers" Eye Movements. Columbus, ohio: Engineering Experiment Sitation, The Ohio State University, Report EES 277-1, Apri1., 1968.

Yarbus, "A.I. Eye Movements and Viston. Translated by L.A. Riggs.. New York: Plenum Press, 1967.

Zell1, J.oIK.; Rockwel1, T.H. and Mourant, R.R. Visual Search in Driviling as a Function of Experience, Columbus, Ohio: Ohio Statte University Driving Research Laboratory, 1969. 


\section{APPENDIX A}

DATA COLLECTIONS SEQUENCES, IMPLIED CONSENT FORM AND

\section{SUBJECTS' STATIC VISUAL AND ANTHROPOMETRIC MEASUREMENTS}

\section{Contents}

Title

Page

Table A.1: Subject 非's Test Sequence

Table A.2: Subject 非's Test Sequence

Table A. 3: Subject \# $^{\text {'s }}$ Test Sequence

115

Table A.4: Subject Implied Consent Form

116

Table A.5: Subjects' Bausch and Lomb Vision Scores

117

Table A.6: Subjects' Anthropometric Measurements

118

Table A.7: Anthropometric Definitions 
Table A.1

Subject \#1's Test Sequence

\begin{tabular}{|c|c|c|c|}
\hline $\begin{array}{c}\text { Data } \\
\text { Collection } \\
\text { Order }\end{array}$ & $\begin{array}{c}\text { Boating } \\
\text { Environment }\end{array}$ & $\begin{array}{c}\text { Navigation } \\
\text { Task }\end{array}$ & $\begin{array}{c}\text { Velocity } \\
\text { Level }\end{array}$ \\
\hline 1 & Limited Access & Compass & Low \\
\hline 2 & Limited Access & Visual Ref. Pt. & Low \\
\hline 3 & Limited Access & Visual Ref. Pt. & Medium \\
\hline 4 & Limited Access & Center in Channel & High \\
\hline$\therefore$ & Limited Access & Center in Channel & Medium \\
\hline$\therefore 6$ & Open & Compass & Low \\
\hline 7 & Open & Visual Ref. Pt. & Medium \\
\hline 8 & Open & Visual Ref. Pt. & Low \\
\hline 9 & Open & Center in Channel & High \\
\hline 10 & Open & Center in Channe1 & Medium \\
\hline 11 & Limited Access & Compass & Medium \\
\hline 12 & Limited Access & Compass & High \\
\hline 13 & Limited Access & Visual Ref. Pt. & High \\
\hline 14 & Limited Access & Center in Channel & Low \\
\hline 15 & Open & Compass & High \\
\hline 16 & Open & Compass & Medium \\
\hline 17. & Open & Visual Ref. Pt. & High \\
\hline 18 & Open & Center in Channel & Low \\
\hline
\end{tabular}


Table A. 2

Subject 非's Test Sequence

\begin{tabular}{|c|c|c|c|}
\hline $\begin{array}{c}\text { Data } \\
\text { Collection } \\
\text { Order }\end{array}$ & $\begin{array}{c}\text { Boating } \\
\text { Environment }\end{array}$ & $\begin{array}{c}\text { Navigation } \\
\text { Task }\end{array}$ & $\begin{array}{l}\text { Velocity } \\
\text { Leve1 }\end{array}$ \\
\hline 1 & Limited Access & Visual Ref. Pt. & Low \\
\hline 2 & Limited Access & Center in Channel & Medium \\
\hline 3 & Open & Compass & High \\
\hline 4 & Open & Compass & Medium \\
\hline 5 & Open & Visual Ref. Pt. & Low \\
\hline 6 & Open & Visual Ref. Pt. & Medium \\
\hline 7 & Open & Center in Channel & Low \\
\hline 8 & Limited Access & Compass & Medium \\
\hline 9 & Limited Access & Compass & High \\
\hline 10 & Limited Access & Visual Ref. Pt. & High \\
\hline 11 & Limited Access & Visual Ref. Pt. & Medium \\
\hline 12 & Limited Access & Center in Channel & High \\
\hline 13 & Limited Access & Center in Channel & Low \\
\hline 14 & Open & Compass & Low , \\
\hline 15 & Open & Visual Ref. Pt. & High \\
\hline 16 & Open & Center in Channel & Medium \\
\hline 17 & Open & Center in Channel & High \\
\hline 18 & Limited Access & Compass & Low \\
\hline
\end{tabular}


Table A,3

Subject 非's Test Sequence

\begin{tabular}{|c|c|c|c|}
\hline $\begin{array}{c}\text { Data } \\
\text { Collection } \\
\text { Order }\end{array}$ & $\begin{array}{c}\text { Boating } \\
\text { Environment }\end{array}$ & $\begin{array}{c}\text { Navigation } \\
\text { Task }\end{array}$ & $\begin{array}{l}\text { Velocity } \\
\text { Level }\end{array}$ \\
\hline 1 & Open & Compass & High \\
\hline 2 & Open & Visual Ref. Pt. & High \\
\hline 3 & Open & Visual Ref. Pt. & Low \\
\hline 4 & Open & Center in Channel & Low \\
\hline 5 & Open & Center in Channel & Medium \\
\hline$\because$ & Limited Access & Compass & Medium \\
\hline 7 & Limited Access & Visual Ref. Pt. & Low \\
\hline 8 & Limited Access & Visual Ref. Pt. & Medium \\
\hline 9 & Limited Access & Center in Channel & High \\
\hline 10 & Limited Access & Center in Channel & Medium \\
\hline 11 & Open & Compass & Low \\
\hline 12 & Open & Compass & Medium \\
\hline 13 & Open & Visual Ref. Pt. & Medium \\
\hline 14 & Open & Center in Channel & High \\
\hline 15 & Limited Access & Compass & Low \\
\hline 16 & Limited Access & Compass & High \\
\hline 17 & Limited Access & Visual Ref. Pt. & High \\
\hline 18 & Limited Access & Center in Channel & Low \\
\hline
\end{tabular}


Table A.4

Subject Implied-Consent Form

I, the undersigned, understand that the purpose of this study is to determine basic information about the visual behavior and body movements of boat operators. Specific tests in which I will be asked to be a subject include: (a) anthropometric measurements, (b) static visual measurements such as visual acuity, and (c) measurements of eye movements and eye fixation locations. I acknowledge that I have received a complete briefing of these tests and I am satisfied that I understand what is involved. I know of no physical disabilities which would prevent me from taking part in this experiment. I realize some discomfort could result from my participation although the experimental procedures and apparatus have been designed to minimize these hazards. I also understand that my participation is strictly voluntary and that I will be allowed, at any time, to stop for rest or to discontinue my participation in this study without prejudice or change in my pay. I further acknowledge that all of the data are confidential and I agree to allow publication of any or all of the data collected if presented in a coded form not identifying me. 
Table A.5

Subjects' Bausch and Lomb Vision Scores

\begin{tabular}{|c|c|c|c|}
\hline Measurement & Subject 非1 & Subject 非 & Subject \#3 \\
\hline $\begin{array}{l}\text { Far Vision: } \\
\text { Vertical Phoria (Prism Diopters) } \\
\text { Lateral Phoria (Prism Diopters) } \\
\text { Acuity-Both Eyes (Snellen } \\
\text { Fraction) } \\
\text { Acuity-Right Eye (Snellen } \\
\text { Fraction) } \\
\text { Acuity-Left Eye (Snellen } \\
\text { Fraction) } \\
\text { Depth Perception (\% Stereopsis) } \\
\text { Color Vision } \\
\text { Near Vision: } \\
\text { Vertical Phoria (Prism Diopters) } \\
\text { Lateral Phoria (Prism Diopters) } \\
\text { Acuity-Both Eyes (Snellen } \\
\text { Fraction) } \\
\text { Acuity-Right Eye (Snellen } \\
\text { Fraction) } \\
\text { Acuity-Left Eye (Snellen } \\
\text { Fraction) }\end{array}$ & $\begin{array}{l}0.5 \mathrm{LH} \\
+1.33 \\
20 / 20 \\
20 / 18 \\
20 / 20 \\
102.4 \% \\
\text { Below } \\
\text { Standard }\end{array}$ & $\begin{array}{l}0.5 \mathrm{LH} \\
-1.66 \\
20 / 18 \\
20 / 25 \\
\\
20 / 17 \\
103.6 \% \\
\text { Satisfac- } \\
\text { tory }\end{array}$ & $\begin{array}{l}0.5 \mathrm{LH} \\
+1.33 \\
20 / 18 \\
20 / 17 \\
20 / 17 \\
96.0 \% \\
\text { Satisfac- } \\
\text { tory }\end{array}$ \\
\hline
\end{tabular}

${ }^{1}$ Standard used was the Ortho-Rater Visual Performance Profile for Operators of Mobile Equipment. 
Table A. 6

Subjects' Anthropometric Measurements ${ }^{1}$

\begin{tabular}{|lccc|}
\hline Measurement $^{2}$ & Subject \#1 & Subject \#2 & Subject \#3 \\
\hline Sitting Height & $82.3(32.4)$ & $89.7(35.3)$ & $91.4(36.0)$ \\
Seated Eye Height & $72.1(28.4)$ & $80.0(31.5)$ & $80.0(31.5)$ \\
Shoulder Height & $54.6(21.5)$ & $62.2(24.5)$ & $66.0(26.0)$ \\
E1bow Rest Height & $18.8(7.4)$ & $22.9(9.0)$ & $27.9(11.0)$ \\
Shoulder Width & $42.7(16.8)$ & $44.2(17.4)$ & $45.7(18.0)$ \\
Upper Arm Length & $34.5(13.6)$ & $36.8(14.5)$ & $36.8(14.5)$ \\
Lower Arm Length & $44.7(17.6)$ & $45.7(18.0)$ & $47.8(18.8)$ \\
Poplitea1 Length & $4.0 .1(15.8)$ & $44.5(17.5)$ & $43.9(17.3)$ \\
Popliteal Height & $44.2(17.4)$ & $43.2(17.0)$ & $44.5(17.5)$ \\
Knee Height & $52.6(20.7)$ & $55.9(22.0)$ & $57.2(22.5)$ \\
Height & $163.3(64.3)$ & $172.7(68.0)$ & $182.4(71.8)$ \\
Weight (kg \& 1bs) & $59.9(132)$. & $70.3(155)$. & $79.4(175)$. \\
Age (years) & 28 & 21 & 20 \\
\hline
\end{tabular}

${ }^{1}$ Measurements defined in Table A.7

2Measurements in centimeters with inches in parentheses unless otherwise indicated. All measurements were taken with a yardstick and tape measure; except for weight, where the subjects stated their weight. 
Table A.7

Anthropometric Definitions

\begin{tabular}{|c|c|}
\hline Measurement & Definition \\
\hline Sitting Height & $\begin{array}{l}\text { Subject sits erect, his head in a Frankfort } \\
\text { plane. Measurement is taken from the sitting } \\
\text { surface to the top of the head. }\end{array}$ \\
\hline Seated Eye Height & $\begin{array}{l}\text { Subject sits erect, his head in a Frankfort } \\
\text { plane. Eye height is measured as the distance } \\
\text { from the sitting surface to the inner corner } \\
\text { (internal canthus) of the right eye. }\end{array}$ \\
\hline Shoulder Height & $\begin{array}{l}\text { Subject sits erect. Measurement is taken from } \\
\text { the sitting surface to the right acromion } \\
\text { (highest point on the lateral edge of the } \\
\text { shoulder bone). }\end{array}$ \\
\hline Elbow Rest Height & $\begin{array}{l}\text { Subject sits erect, his right upper arm hanging } \\
\text { at his side with his lower arm extended } \\
\text { horizontally. Measurement is taken from the } \\
\text { sitting surface to the bottom of the right } \\
\text { elbow. }\end{array}$ \\
\hline Shoulder Width & $\begin{array}{l}\text { Subject sits erect. Measurement is the hori-. } \\
\text { zontal distance across the shoulders to the } \\
\text { maximum lateral protusion of the deltoids. }\end{array}$ \\
\hline Upper Arm Length & $\begin{array}{l}\text { Subject sits erect, his right upper arm } \\
\text { hanging at his side with his lower arm } \\
\text { extended horizontally. Measurement is the } \\
\text { distance from the bottom of the elbow to } \\
\text { the right acromion. }\end{array}$ \\
\hline Lower Arm Length & $\begin{array}{l}\text { Same position as the upper arm length measure- } \\
\text { ment with fingers extended. Measurement } \\
\text { is the horizontal distance from the tip } \\
\text { of the right elbow to the longest finger. }\end{array}$ \\
\hline Popliteal Length & $\begin{array}{l}\text { Subject sits erect with the upper front } \\
\text { portion of the horizontal sitting surface } \\
\text { lightly touching the back or inside of the } \\
\text { right knee (popliteal area). Measurement is } \\
\text { the distance from the back of the right but- } \\
\text { tocks to the front edge of the sitting. surface. }\end{array}$ \\
\hline
\end{tabular}


Table A.7 (continued)
Measurement
Definition
Popliteal Height
Subject sits erect with the front portion of the horizontal sitting surface lightly touching the underside of the right knee (popliteal area). Measurement is the vertical distance from the top portion of the sitting surface to the surface of the footrest or floor.
Knee Height
Subject sits erect. Measurement is the vertical distance from the surface of the footrest (floor) to the top of the right knee just in back of the patella.
Height
Subject stands erect with his head in a Frankfort plane, heels together and arms hanging at his side. Measurement is from the top of the head to the floor. 
APPENDIX B

CATEGORIES OF TASK RELATED VARIABLES DETERMINED BY DATA REDUCER

\section{CONTENTS}

\section{Title}

Page

Table B.1: Immediate Boating Situation Categories

Table B.2: Maneuvering Situation Categories

Table B.3: Eye Spot Reference Locations

124

Table B.4: Fixation Target Categories

125 
Table B. 1

Immediate Boating Situation Categories

\begin{tabular}{|c|c|c|}
\hline $\begin{array}{l}\text { Code } \\
\text { No. }\end{array}$ & & - Category of Boat \\
\hline & MOVING & BOATS \\
\hline 1 & & $\begin{array}{l}\text { Boat dead ahead within } 250 \text { yards moving } \\
\text { toward us }\end{array}$ \\
\hline 2 & & Boat dead ahead moving away from us \\
\hline 3 & & Boat approaching port \\
\hline 4 & & Boat approaching starboard \\
\hline 5 & & Boat rear starboard \\
\hline 6 & & Boat approaching from stern \\
\hline 7 & & Boat passing port to starboard \\
\hline 8 & & Boat passing starboard to port \\
\hline 9 & & Freighter or Bob-Lo boat ahead \\
\hline 10 & . & Boat rear port \\
\hline 11 & & Freighter and boat port \\
\hline 12 & & Bob-Lo boat port \\
\hline 13 & & Bob-Lo boat starboard \\
\hline 14 & & No traffic within 250 yards \\
\hline$\ldots$ & ANCHORED & BOATS \\
\hline 15 & & Anchored boat port \\
\hline 16 & & Anchored boat starboard \\
\hline 17 & & Anchored boat starboard and port \\
\hline
\end{tabular}


Table B.2

Maneuvering Situation Categories

\begin{tabular}{|cl|}
\hline $\begin{array}{c}\text { Code } \\
\text { No. }\end{array}$ & \multicolumn{1}{c|}{ Maneuvering Situation } \\
\hline 1 & Moving straight - calm water \\
2 & Moving straight - 1ight chop water \\
3 & Moving straight - rough water \\
4 & Going over wake \\
5 & Turning right - calm water \\
6 & Turning right - 1ight chop water \\
7 & Turning right - rough water \\
8 & Turning left - calm water \\
9 & Turning left - light chop water \\
10 & Turning left - rough water \\
\hline
\end{tabular}


Table B.3

Eye Spot Reference Locations

\begin{tabular}{|cl|}
\hline $\begin{array}{c}\text { Code } \\
\text { No. }\end{array}$ & \multicolumn{1}{c|}{ Vertical Reference Location } \\
\hline 1 & Right front bow marker \\
2 & Center front bow marker \\
3 & Center pillar \\
4 & Windshield wiper motor \\
5 & Right front pillar \\
6 & $\begin{array}{c}\text { Left front pillar } \\
7\end{array}$ \\
8 & $\begin{array}{c}\text { Keft side pillar } \\
\text { tachometer, speedometer, compass, or face camera) }\end{array}$ \\
9 & No spot
\end{tabular}

'Horizontal reference was the horizon. 
Table B.4

Fixation Target Categories

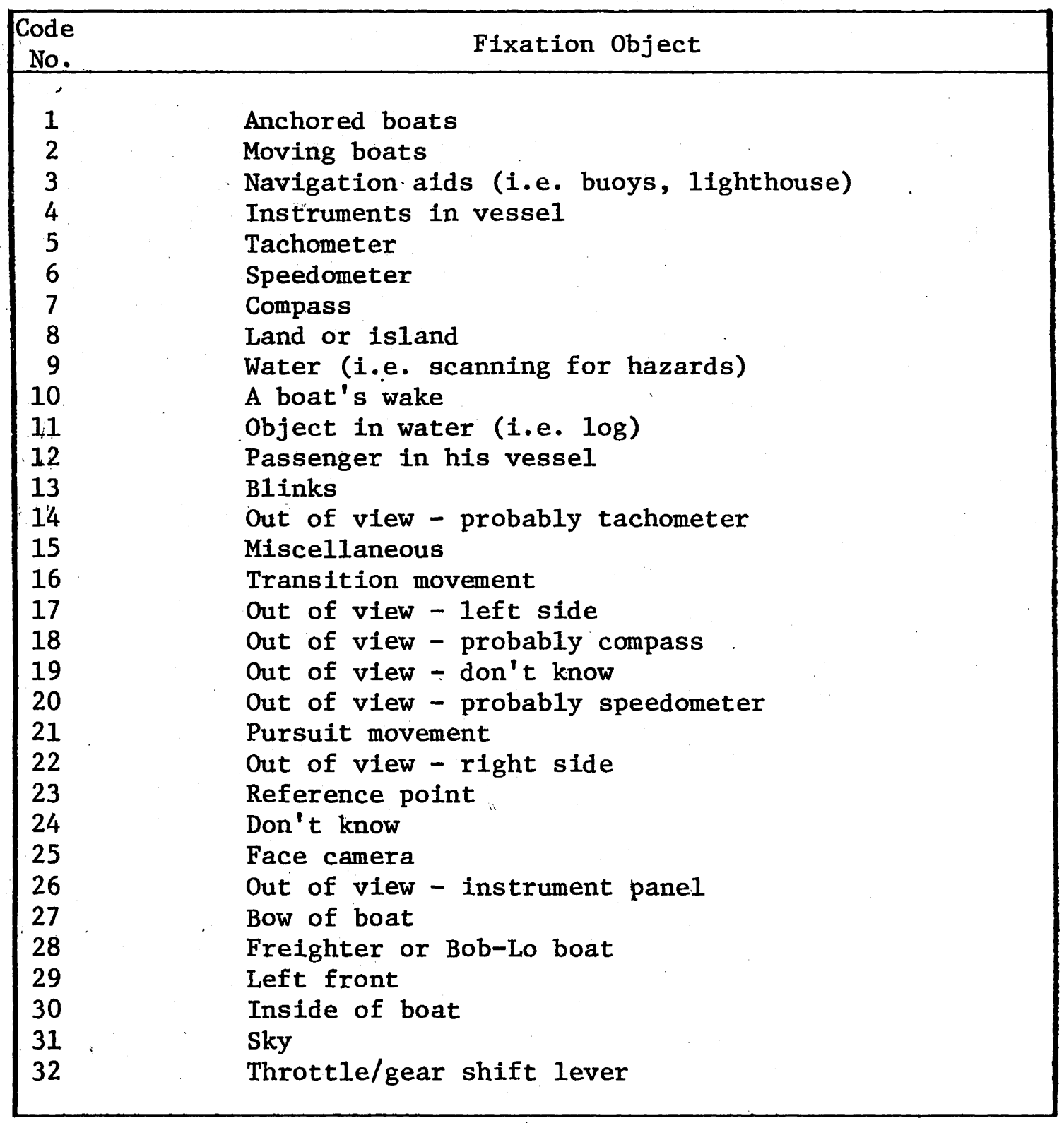




\section{APPENDIX C}

STATISTICAL SUMMARY OF EYE FIXATION DATA

Table C.1: Mean Horizontal Location (degrees) $\quad 127$

Table C.2: Standard Deviation of Horizontal Location (degrees) 128

Table C.3: Mean Vertical Location (degrees) 129

Table C.4: Standard Deviation of Vertical Location (degrees) 130

Table C.5: Fixation Durations (msec) 131 


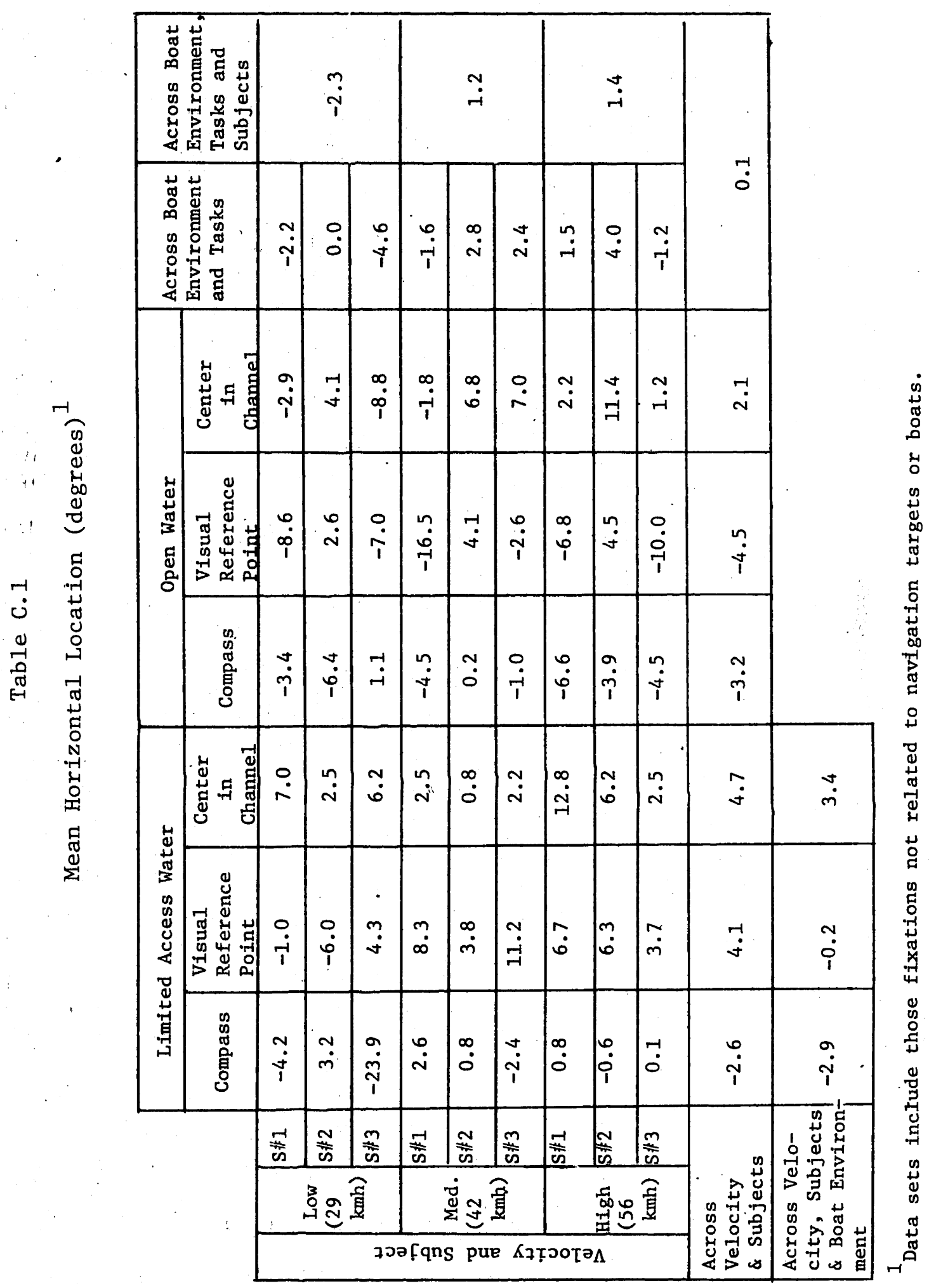




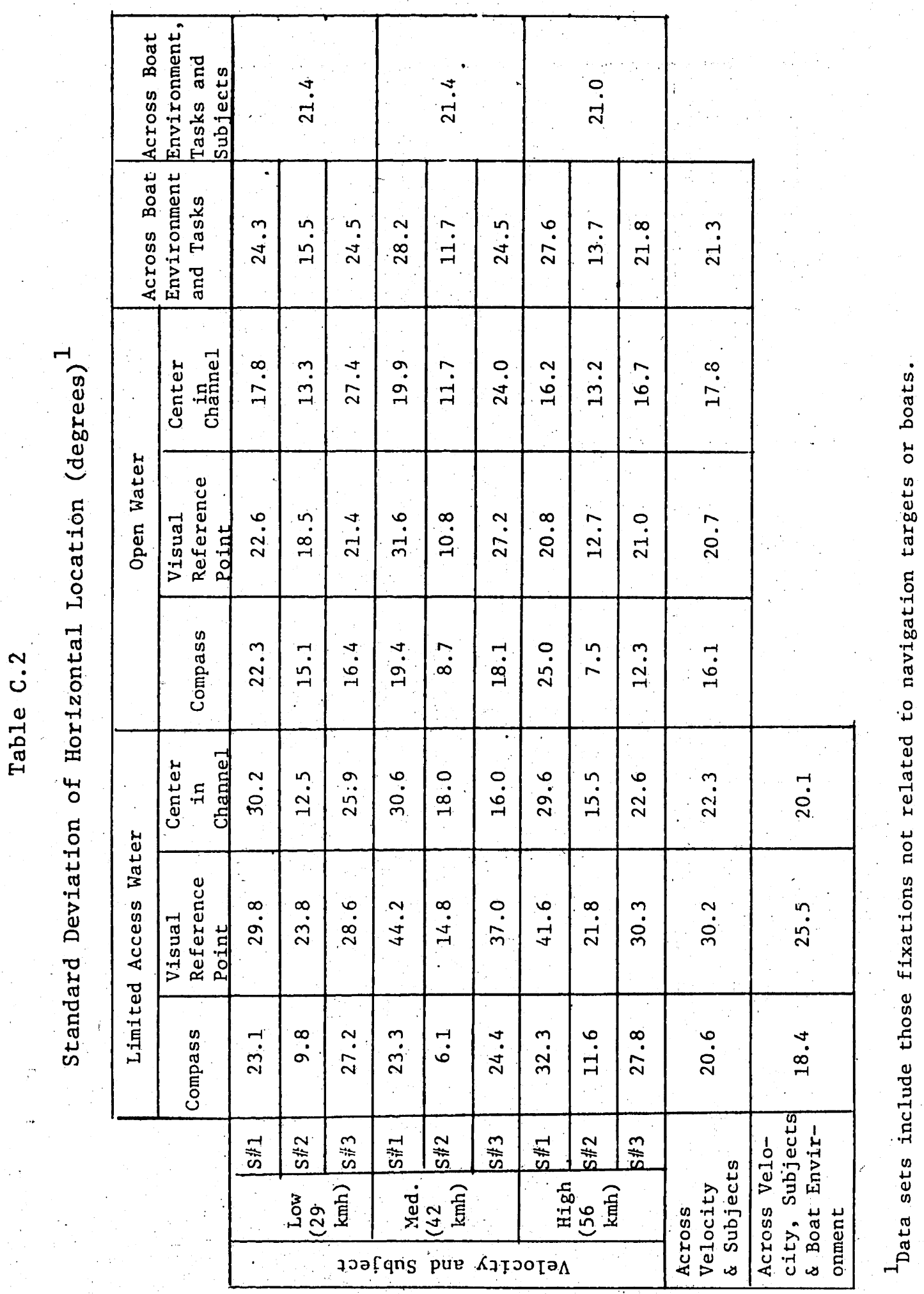




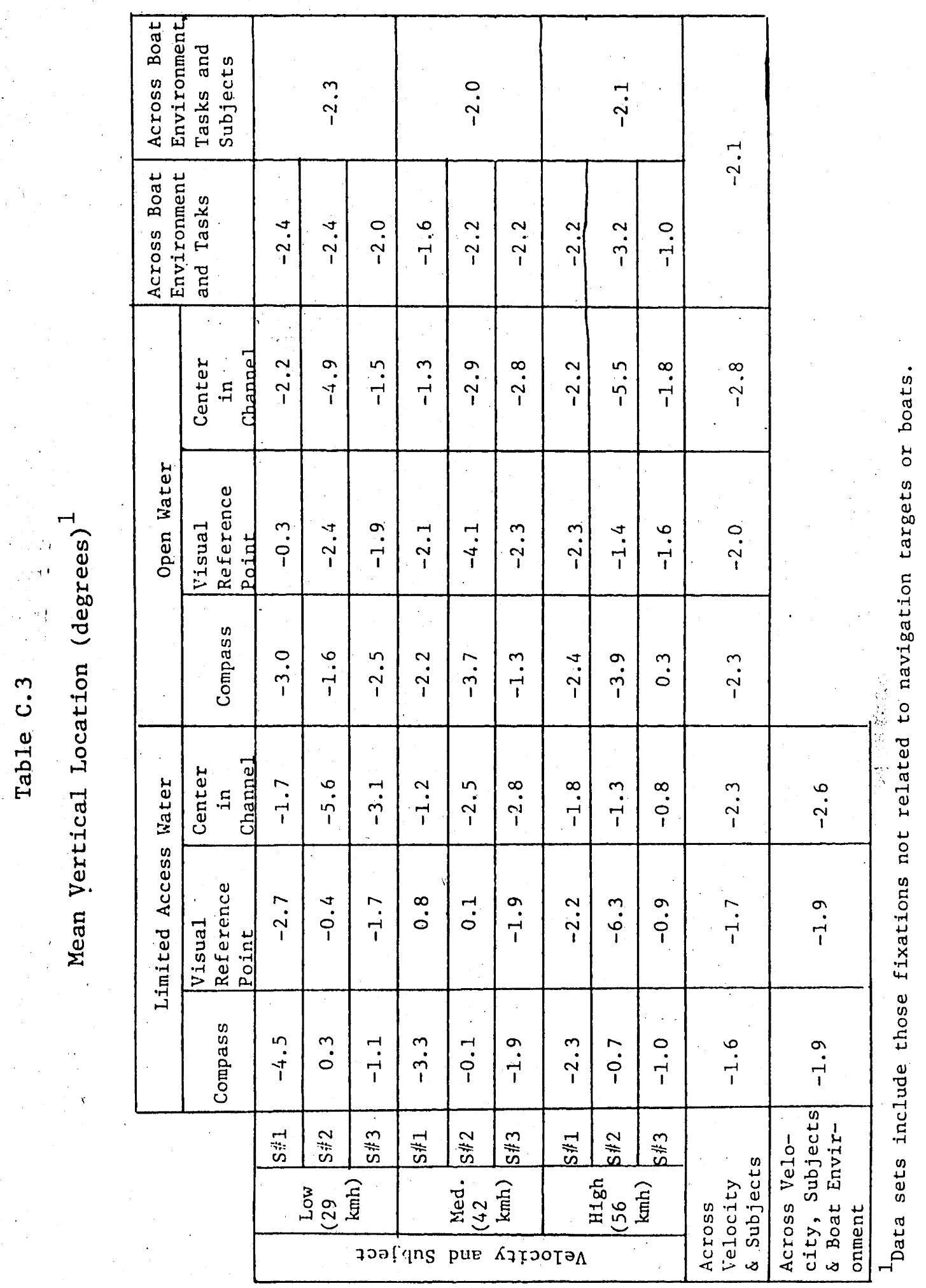




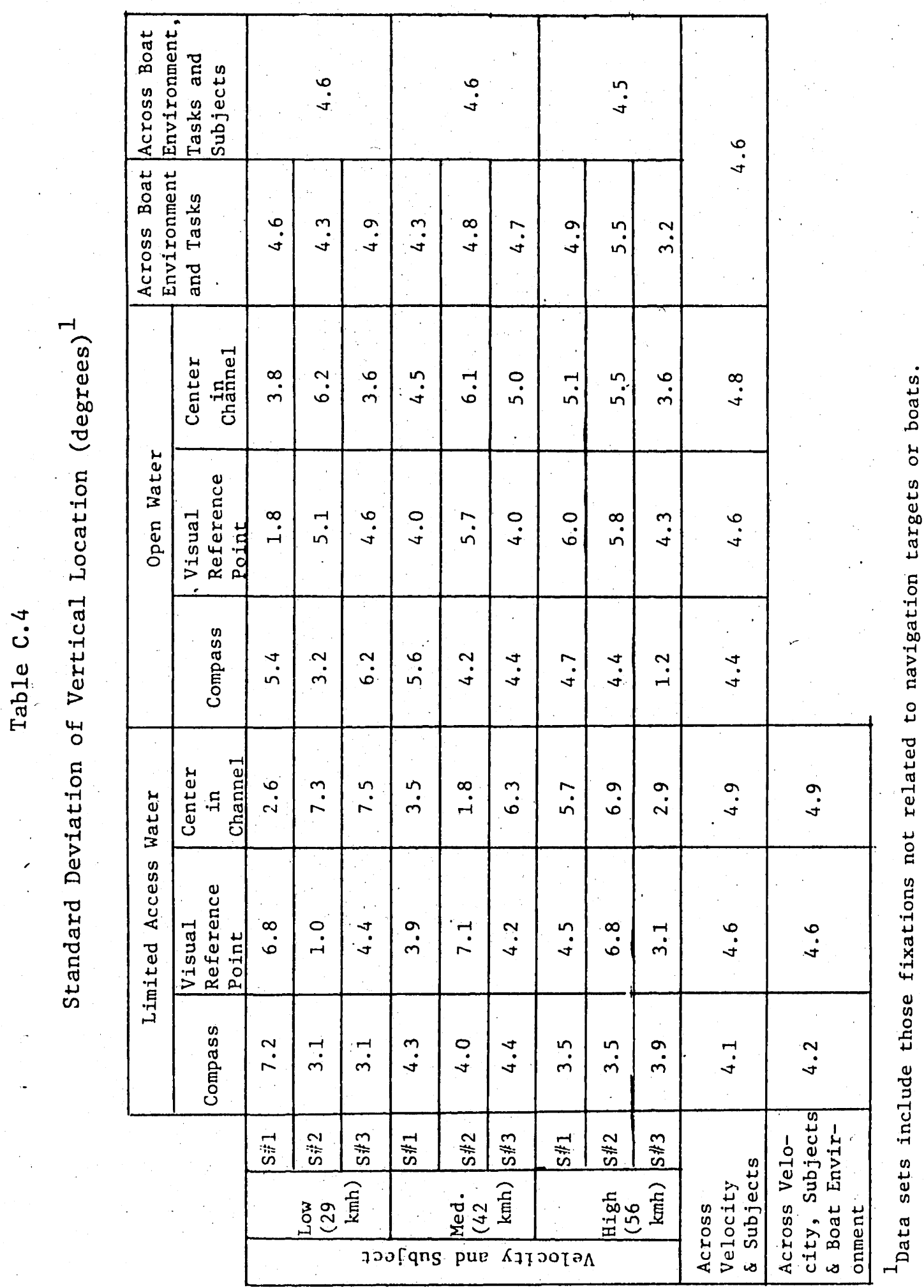




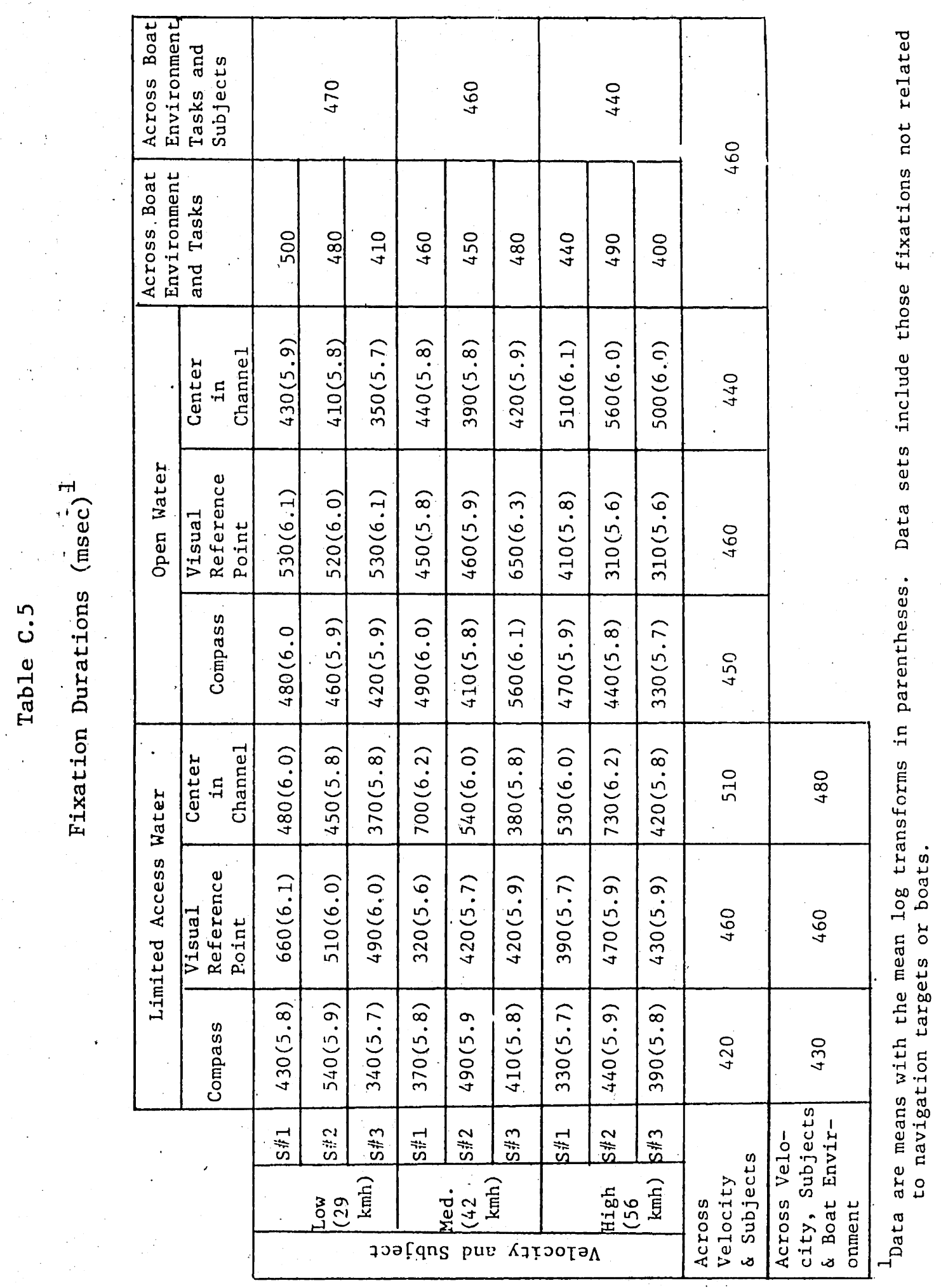




\section{APPENDIX D}

\section{PERCENT FIXATION TIMES. BY VISUAL ZONES}

Tit1e

Page

Definition of Visual Zones

Analysis of Data

Discussion of Results

Left and Right Visual Zones

138

Center Visual Zone

Environment and Navigation Task Effects

Subject-Task Interaction Effects

Subject Velocity Interaction Effect

$\underline{\text { Table }}$

Page

Table D.1: Visual Zone Dimensions

Table D.2: Percent Fixation Time in Left Visual Zone

139

Table D.3: Percent Fixation Time in Center Visual Zone

Table D.4: Percent Fixation Time in Right Visual Zone

141

Table D.5: Significant Results from the Analysis of Variance for Percent Fixation Time in Visual Zones

Figure

Page

Figure D.1: Automotive Visual Zones

Figure D.2: Boating Center Visual Zone as Viewed by the Operator

Figure D.3: Boating Visual Zones

Figure D.4: Subject Effects on Percent Fixation Time in Visual Zones

Figure D.5: Boating Environment Effects on Percent Fixation Time in Visual Zones

Figure D.6: Subject-Boating Environment Effects on Percent Fixation Time in the Right Visual Zone

Figure D.7: Subject-Navigation Task Effects on Percent Fixation Time in the Center Visual Zone

Figure D.8: Subject-Boating Environment Effects on Percent Fixation Time in the Center Visual Zone

Figure D.9: Subject-Velocity Effects on Percent Fixation Time in The Center Visual Zone

Figure D.10: Components of Variance for the Visual Zones 


\section{PERCENT FIXATION TIMES BY VISUAL ZONES}

\section{DEFINITION OF VISUAL ZONES}

The data analyses centered around the spatial and temporal properties of the boating subjects' eye fixations. Combining these two parameters was achieved by (a) dividing the available scanning area into zones and (b) determining the respective percent of fixation time spent in each zone.

Such a method has been used extensively in the automotive eye movement studies performed at Ohio State University. An 1llustration of the automobile segmented areas is contained in Figure D.1. Rockwe11, Overby and Mourant (1968, p. 32) stated that "the seven sections were chosen so as to contain prominent highway features that were believed to be significant sources of information for the driver in controlling his vehicle."1

Criteria to divide up the boater's visual field into zones were based on the different types of tasks that one might expect the boater to perform.

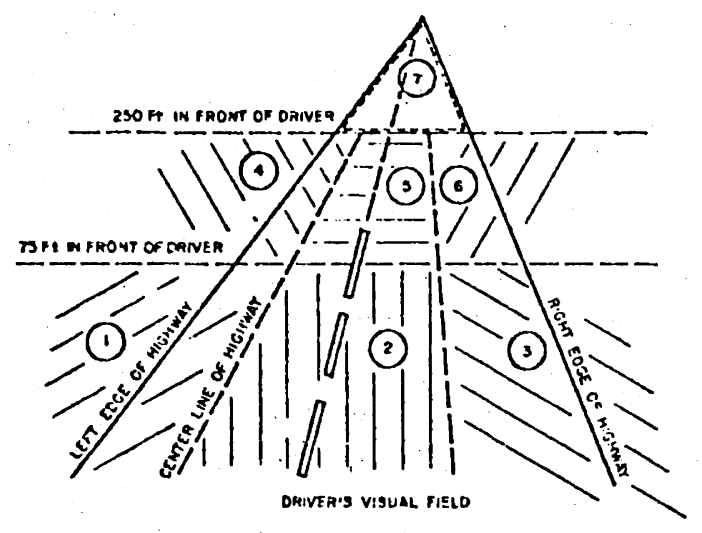

Figure D.1: Automotive Visual Zones (from Rockwe11, Overby and Mourant, 1968, p. 26) 
The primary task of the boater is probably to scan for non-vehicular collision obstacles which may be directly in his path. It was determined that the most prevalent of these areas would be $15^{\circ}$ to either side of straight-ahead. An illustration of this zone is contained in Figure D.2. Other researchers (Bartz, 1965 and Devlin and Roe, 1968) have stated that head movements occur when the visual angle is greater than $30^{\circ}$ to $40^{\circ}$. Boaters scanning within this area would, thus, probably make eye movements without corresponding head movements.

In addition to these front areas, two intermediate type zones were selected to be from $15^{\circ}$ to $45^{\circ}$ right or left of straight-ahead. Boaters scanning in this area could be looking for potential collision vehicles which may come into their path, or non-vehicular collision obstacles which, although not directly in their path, may indicate problem areas (e.g., seaweed or logs on top of the water could indicate shallow areas ahead).

Areas greater than $45^{\circ}$ to $180^{\circ}$ were then encompassed into two more visual zones. Boaters particularly concerned about colliston avoidance would probably more frequently scan these areas to monitor all traffic in their surroundings.

An area straight ahead of the boater $\left( \pm 15^{\circ}\right.$ azimuth) but above the horizon was segmented to account for the scanning of boats, navigation aids or high objects directly in the boater's path. The side areas greater than $\pm 15^{\circ}$ but above the horizon were also portioned. Finally, the instrument panel area including the compass was combined into one zone. 


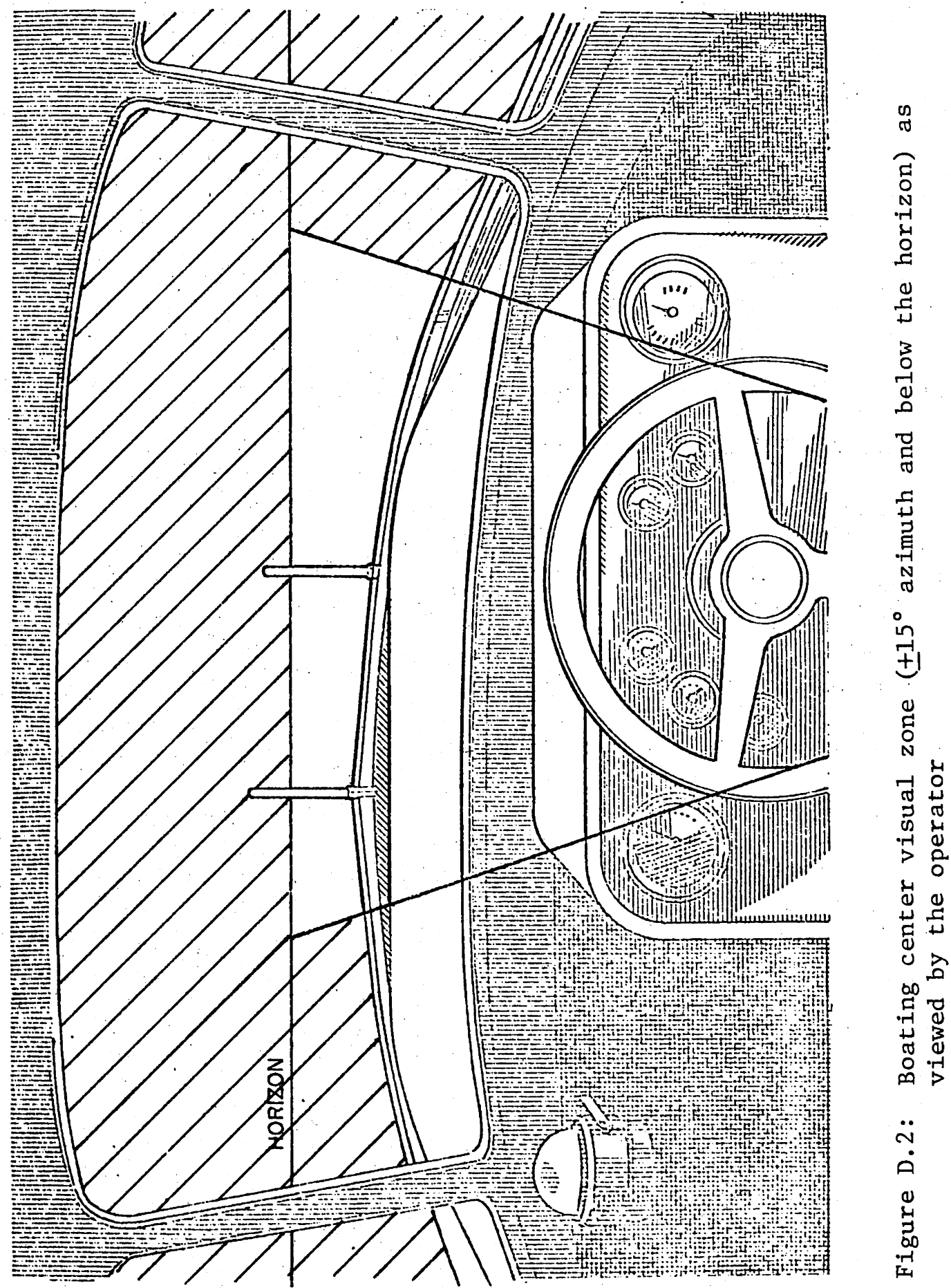


An illustration of all the visual zone segments is contained in Figure D.3. The amount of data collected in this exploratory study. was not sufficient to have fixations in each of the zones. Therefore, zones were recombined into a left visual zone which included visual zones 2,3 , and $7\left(-180^{\circ}\right.$ to $-15^{\circ}$ azimuth); a center visual zone which included zones 4 and $8\left(-15^{\circ}\right.$ to $15^{\circ}$ azimuth); and a right visual zone which included visual zones 5,6 and $9\left(15^{\circ}\right.$ to $180^{\circ}$ azimuth). Dimensions for the original visual zones and the combined zones are contained in Table D.1. Future research on boaters' eye fixations will, hopefully, collect sufficient data for analyses to be possible in the original nine visual zones.

\section{ANALYSIS OF DATA}

The percent of time the boaters spent fixating in each of these zones was determined. (See Tables D.2 - D.4.) Because percentage data is bounded at 0 and $100 \%$, these data were transformed with an arcsin function to obtain an appropriate distribution for the ANOVA analyses. The results from the analyses of variances using the arcsin transforms are contained in Table D.5. The discussion of results will, however, use the percent fixation time numbers rather than their transformed counterparts. 


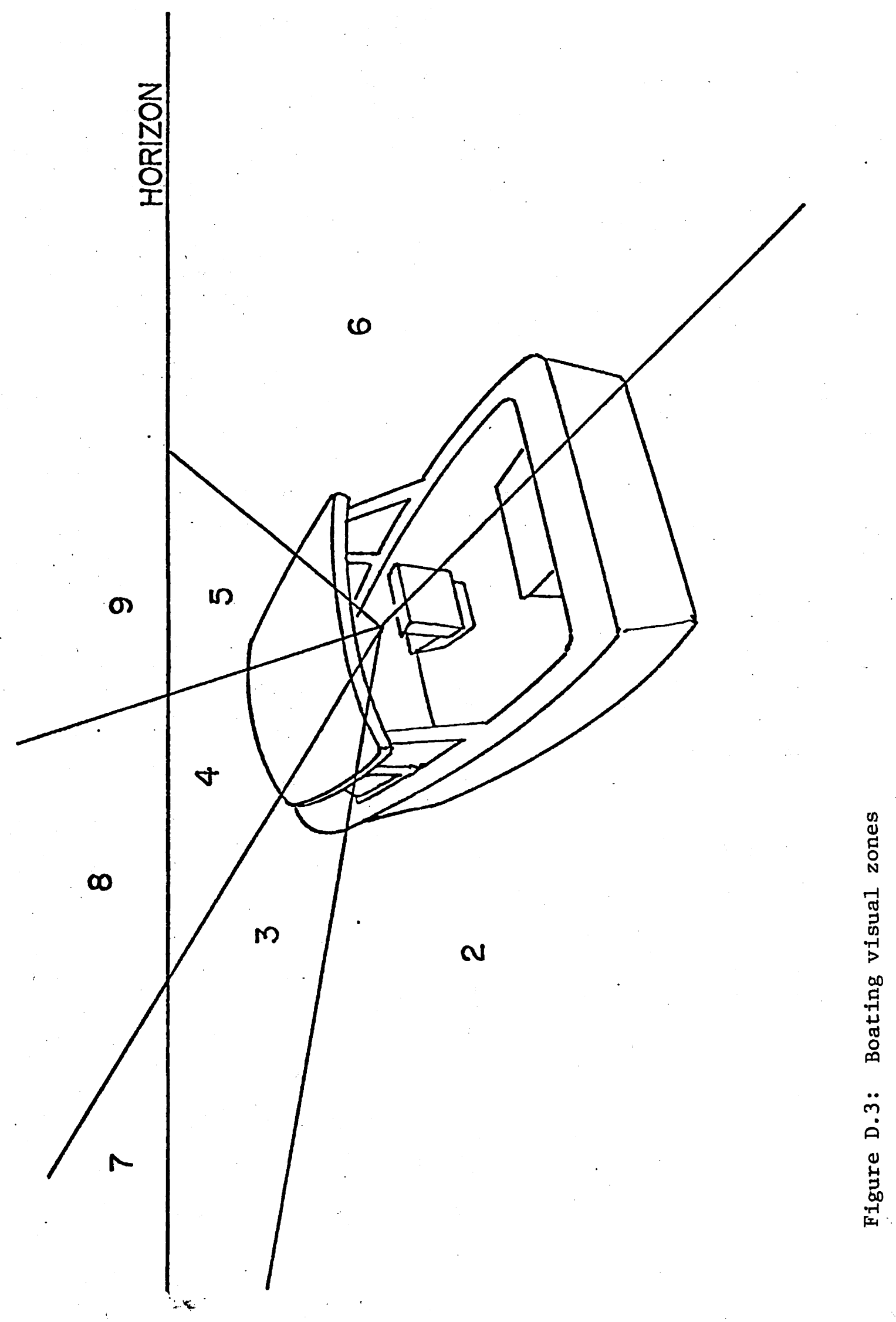


Table D.1

Visual Zone Dimensions

\begin{tabular}{|c|c|c|}
\hline $\begin{array}{c}\text { Visual } \\
\text { Zione No. }\end{array}$ & Dimensions & $\begin{array}{c}\text { Combined Visual } \\
\text { Zone Segment }\end{array}$ \\
\hline 1 & Instrument Panel and Compass & Instrument Panel Zone \\
2 & $-180^{\circ}$ to $-45^{\circ}$ azimuth, below horizon & Left Visual Zone \\
3 & $-45^{\circ}$ to $-15^{\circ}$ azimuth, below horizon & \\
4 & $-15^{\circ}$ to $15^{\circ}$ azimuth, below horizon & Center Visual Zone \\
5 & $15^{\circ}$ to $45^{\circ}$ azimuth, below horizon & Right Visual Zone \\
6 & $45^{\circ}$ to $180^{\circ}$ azimuth, below horizon & Reft Visual Zone \\
7 & $-180^{\circ}$ to $-15^{\circ}$ azimuth, above horizon & Lefor \\
9 & $-15^{\circ}$ to $15^{\circ}$ azimuth, above horizon & Center Visual Zone \\
\hline 9 & $15^{\circ}$ to $180^{\circ}$ azimuth; above horizon & Right Visual Zone \\
\hline
\end{tabular}

\section{DISCUSSION OF RESULTS}

\section{Left and Right Visual Zones.}

Subject differences (S) for all zones were highly significant at $\alpha<.005$ (see Table D.3). This effect was primarily due to Subject 非. Whereas Subjects $\|_{1}$ and $\# 3$ spent approximately $30 \%$ of their time in the left and right visual zones, Subject $\# 2$ only spent $12 \%$ of his time in these side zones (Figure D.4). It is interesting to note from Table D.3 that for the left visual zone, only the subfect variables (S) is significant; however, the right and center visual zones have many variables of significance. 


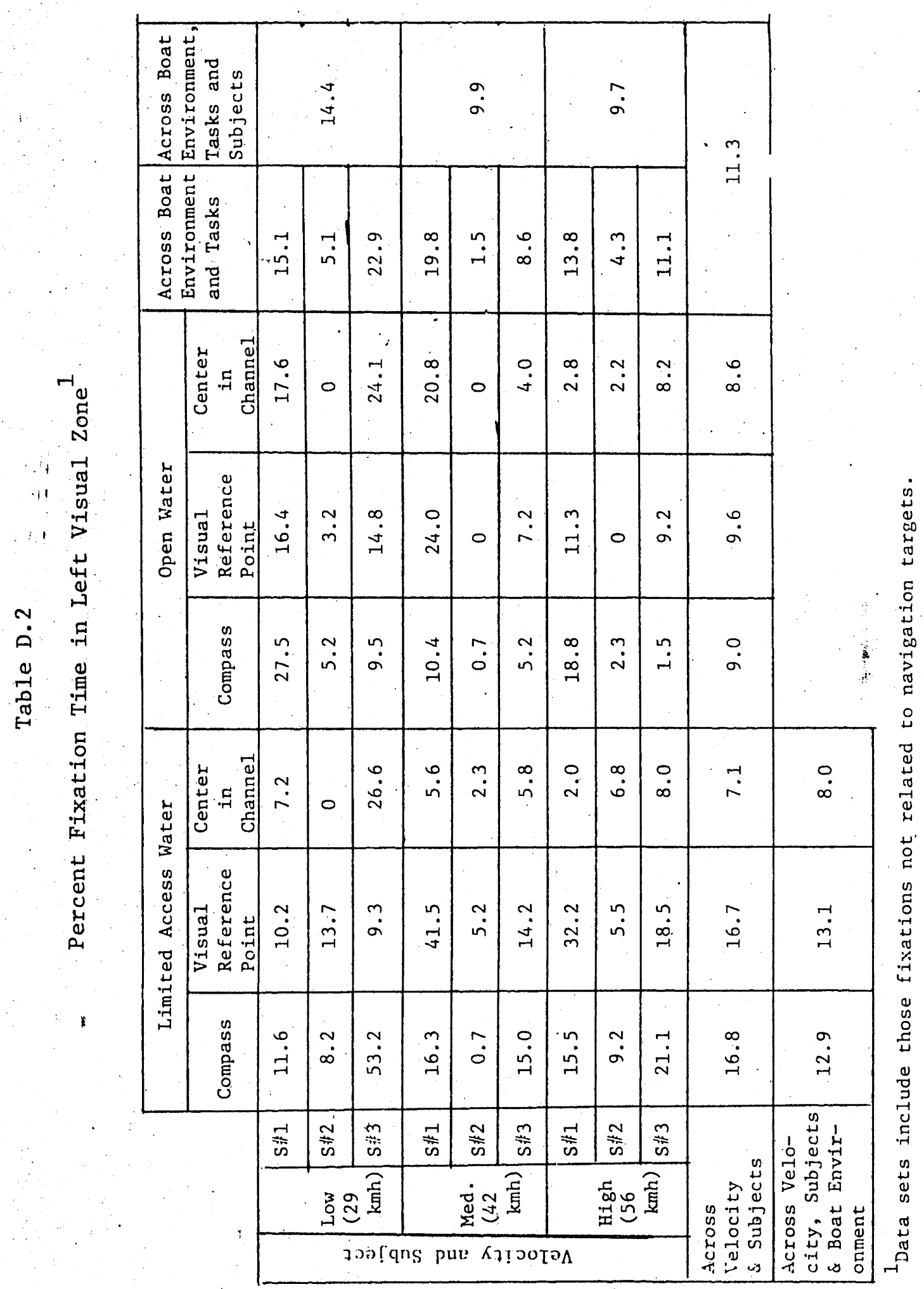




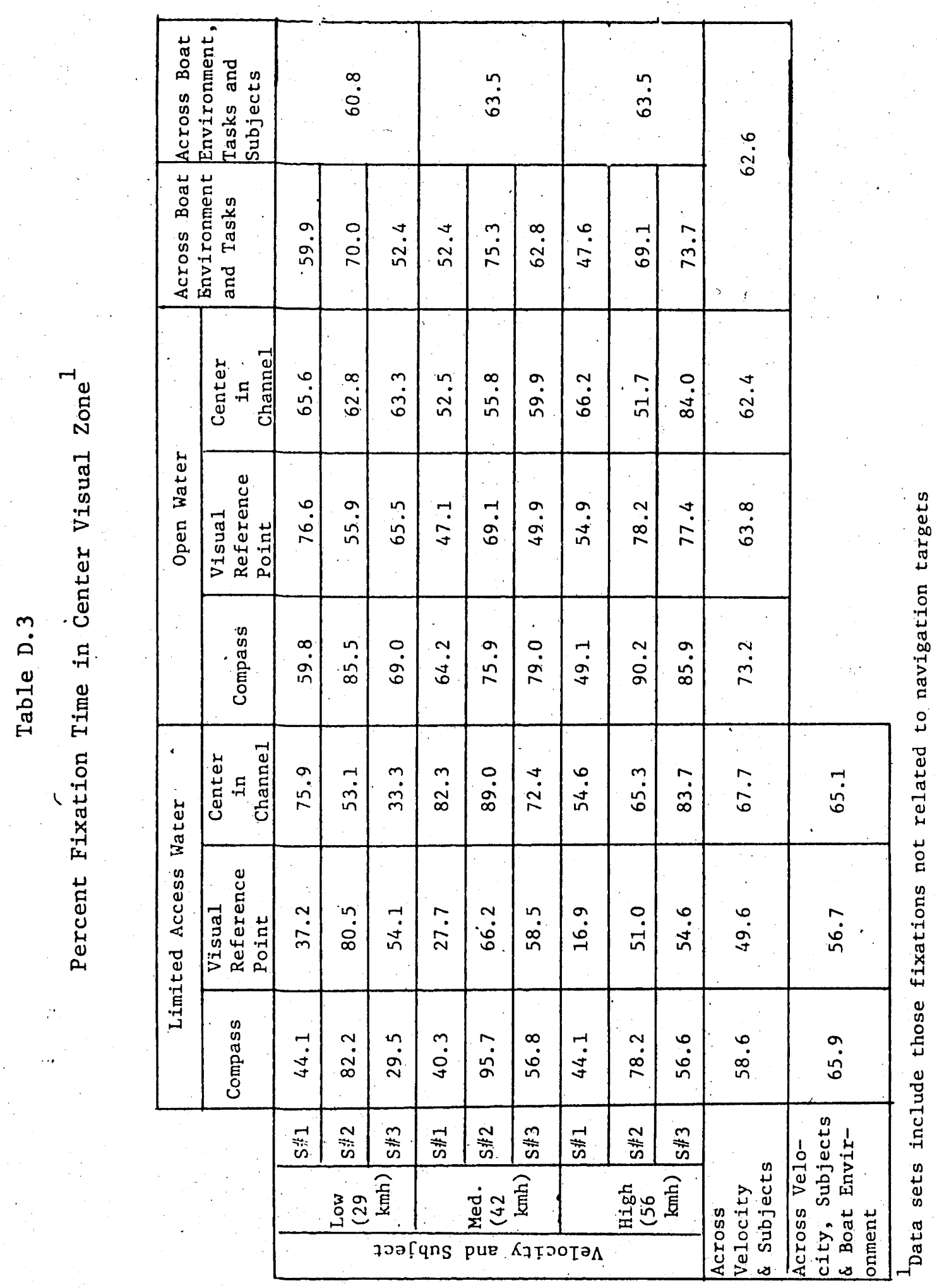




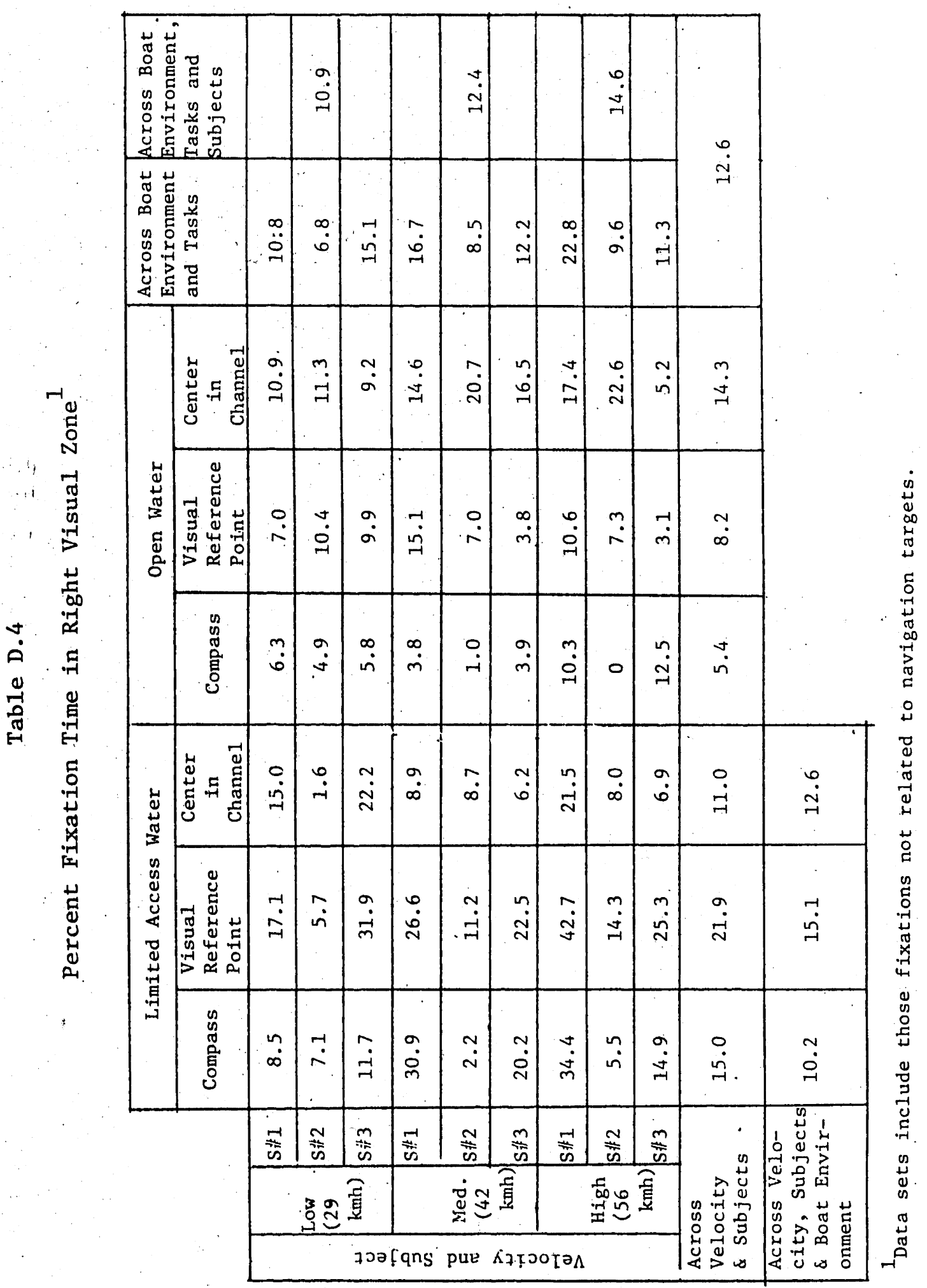


Table D. 5

Significant Results from the Analysis of Variance for Percent Fixation Time in Visual Zones

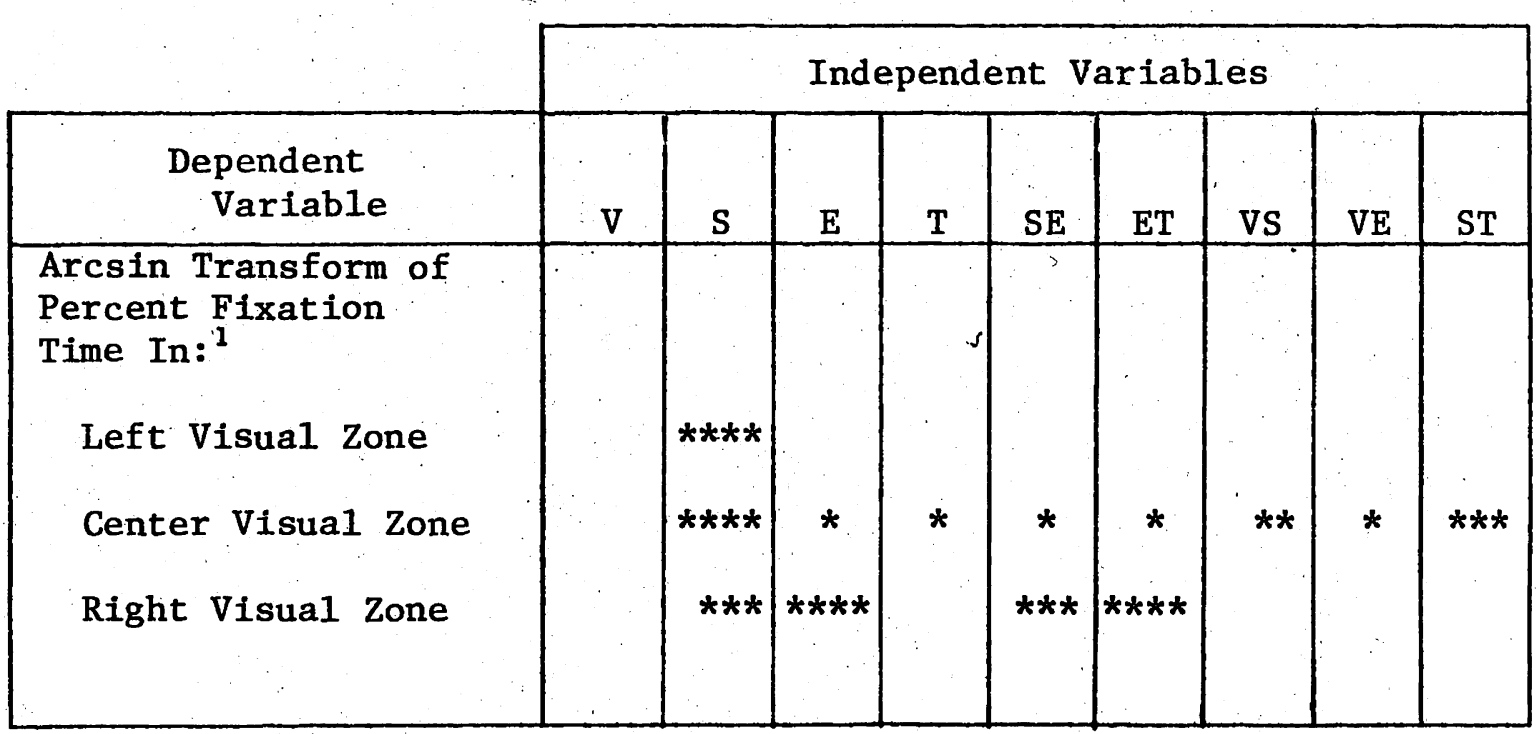

${ }^{1}$ Data sets contain only those fixations not on a particular navigation target

where: $\mathrm{V}=$ Velocity

S = Subject

$\mathrm{E}=$ Boating Environment

$T=$ Navigation Task

$$
\begin{aligned}
& * \quad=\alpha<.05 \\
& * * \quad=\alpha<.01 \\
& * * *=\alpha<.005 \\
& * * * *=\alpha<.001
\end{aligned}
$$




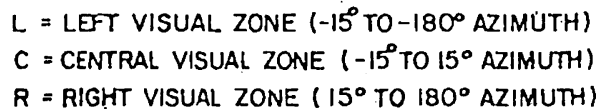

$R=R I G H T$ VISUAL ZONE ( $15^{\circ}$ TO $180^{\circ}$ AZIMUTH)

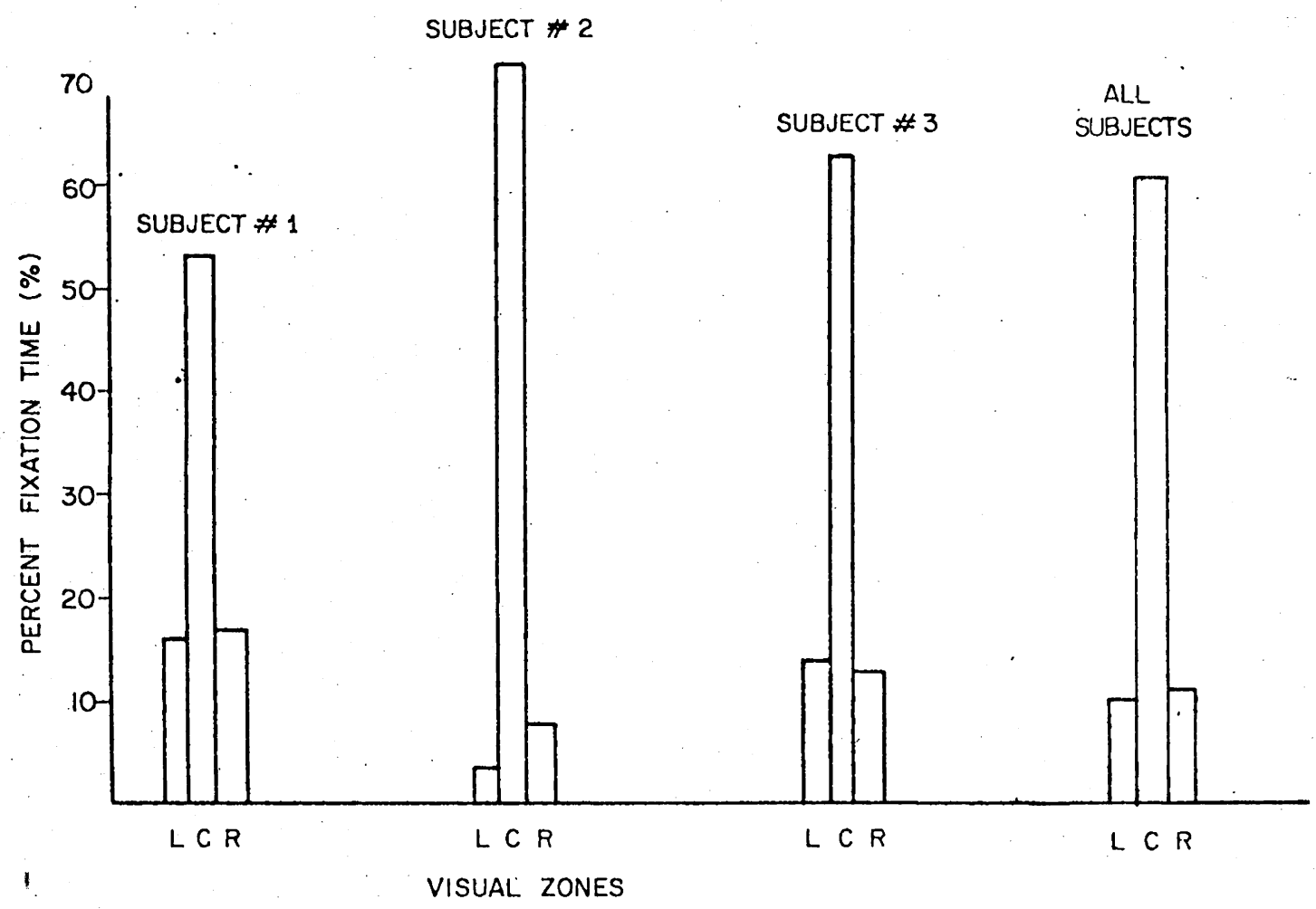

Figure D.4: Subject effects on percent fixation time in visual zones 
With respect to this right zone in particular, the limited access water situation, the boaters spent $16 \%$ of their time fixating in this right zone and only $9 \%$ when they were in open water (see Figure D.5). Furthermore, the subject-boating environment interactions (SE) caused a significant effect on the percent fixation time in this right visual zone (Figure D.6). Both Subjects $\# 1$ and $\# 3$ spent approximately $10 \%$ more time in this zone during the limited access water condition; while Subject 非 2 spent a nonsignificant $2 \%$ more of his time in this right visual zone during the open water condition.

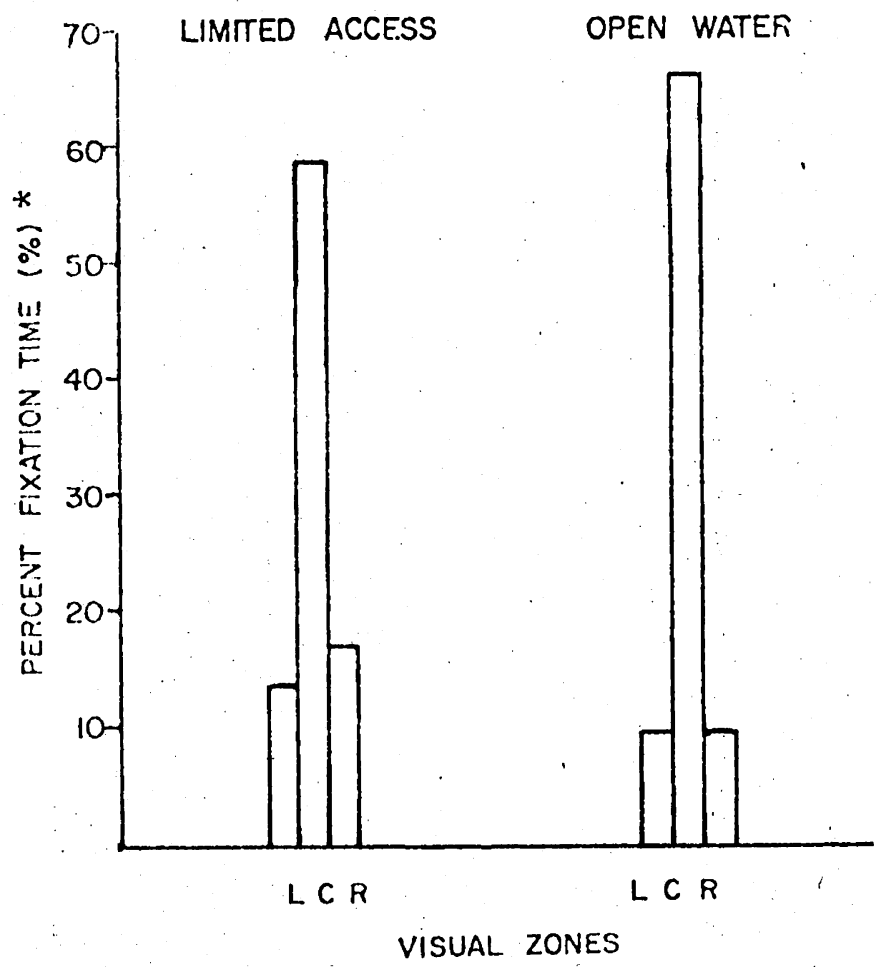

Figure D.5: Boating environment effects on percent fixation time in visual zones (*means for all subjects, all velocities and a11 navigation tasks) 


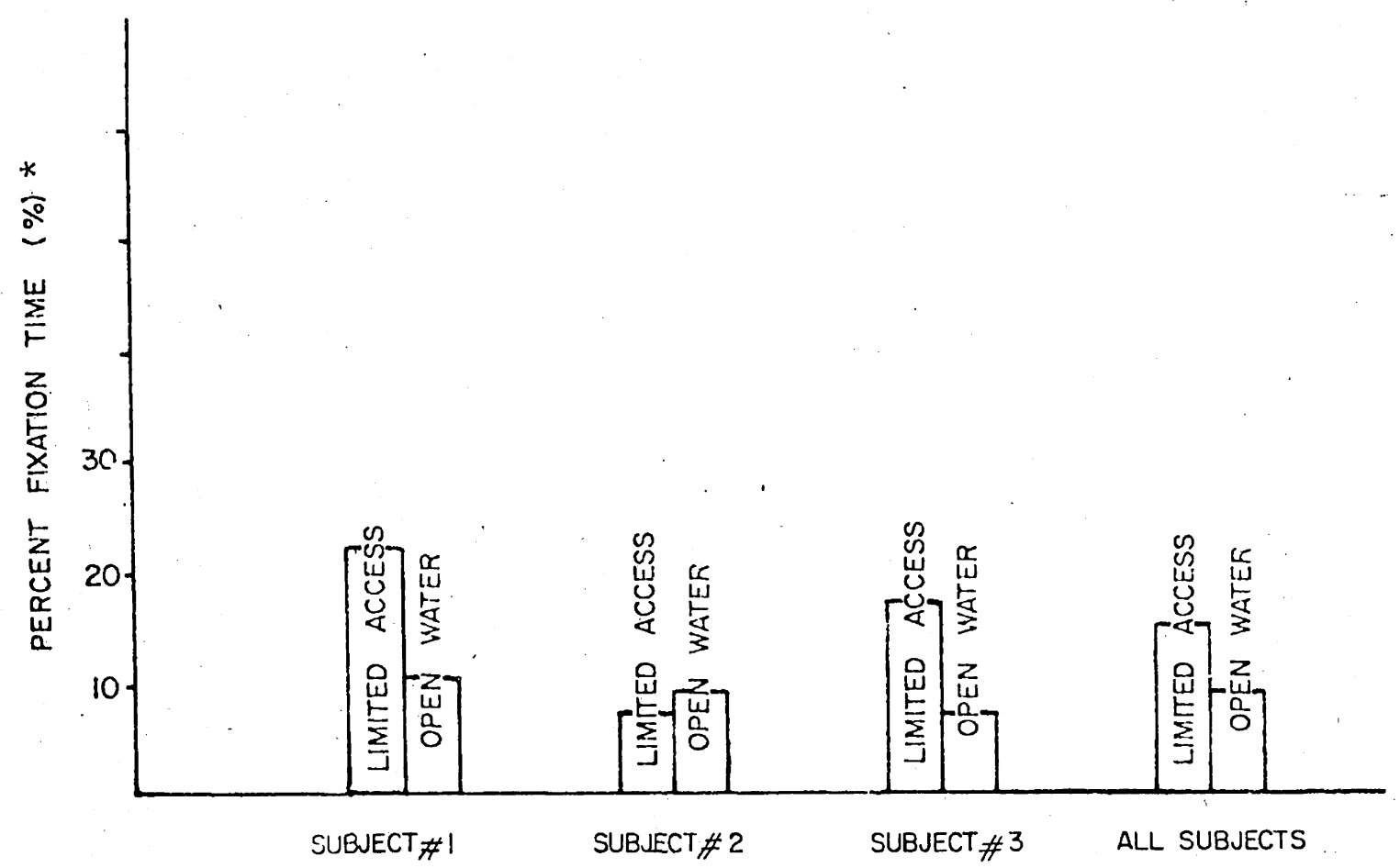

Figure D.6: Subject-boating environment effects on percent fixation time in the right visual zone (*means for all velocities and navigation tasks)

\section{Center Visual Zone}

Environment and Navigation Task Effects

Table D.2 indicates that, overall, less time was spent in this center zone during the limited access water environment than during the open water environment. It was suggested that this tendency might be related to the boater's use of information on his right for purposes of determining his lateral position and tracking error.

With respect to navigation tasks, approximately the same amount of time (65\%) was spent in this center visual zone during the compass and channel tasks; whereas during the visual reference task, on 1 y $57 \%$ of the time was spent fixating to this zone (see Figure. D.7). 


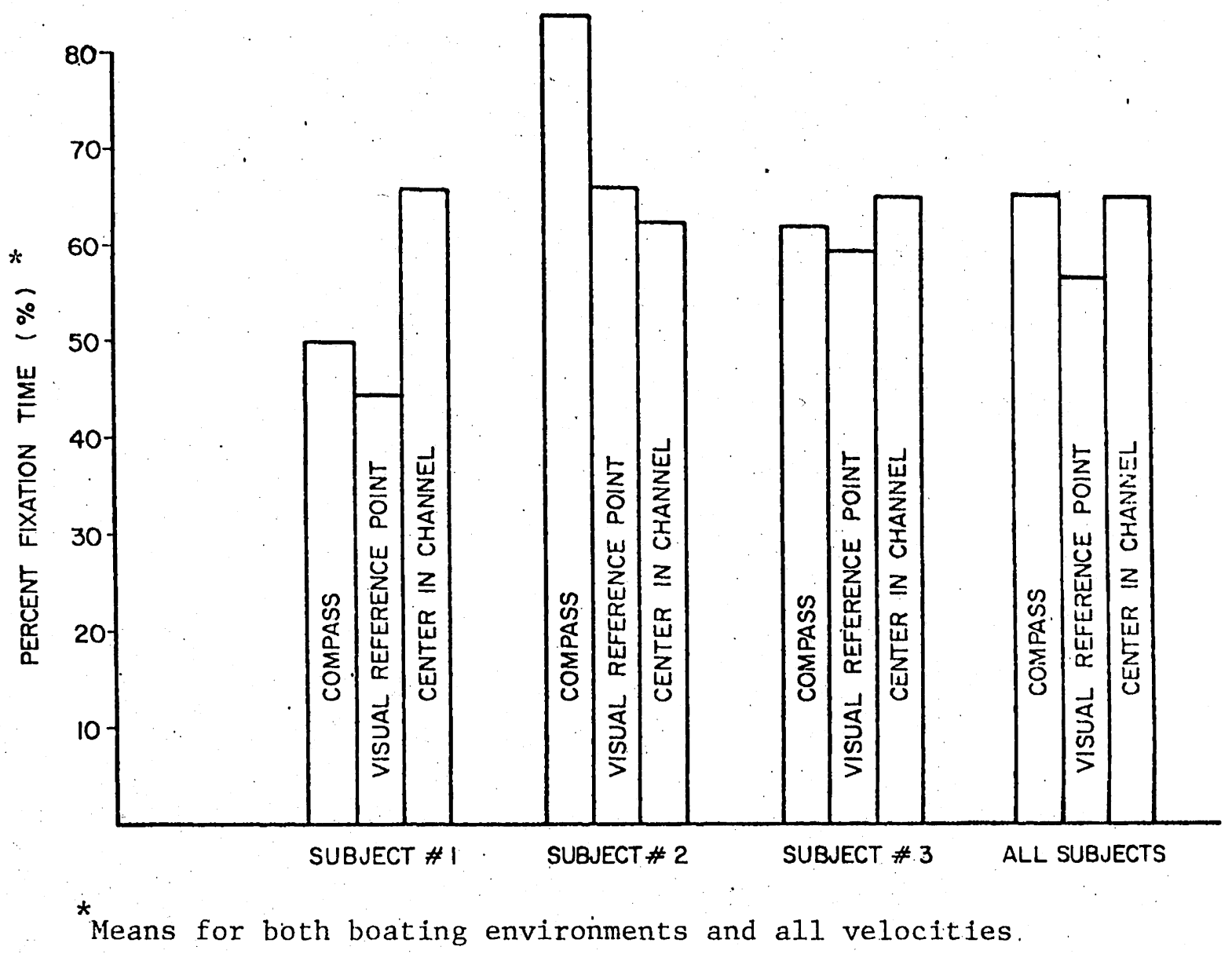

Figure D.7: Subject-navigation task effects on percent fixation time in the center visual zone

\section{Subject-Task Interaction Effects}

Subject-navigation task effects. (ST) were also significant in the Table D. 3 ANOVA's. Subject 3 was consistent in hi.s percent fixation time scross all tasks. (see Figure D.7). Subject $\left.\right|^{2}$ had significantly higher times for the compass task. This is just opposite to Subject 非 who had significantly lower times for the compass task and also for the visual reference point task. A11 subjects had equivalent percent times for the channel tasks. 
Another subject interaction was significant when one considers the boating environment as 11lustrated in Figure D.8. Both Subjects \# 1 and 3 spent less time fixating in the central area during the limited access water condition than they did in the open water condition. Subject $\# 2$, on the other hand, spent approximately the same amount of time regardless of boating environment.

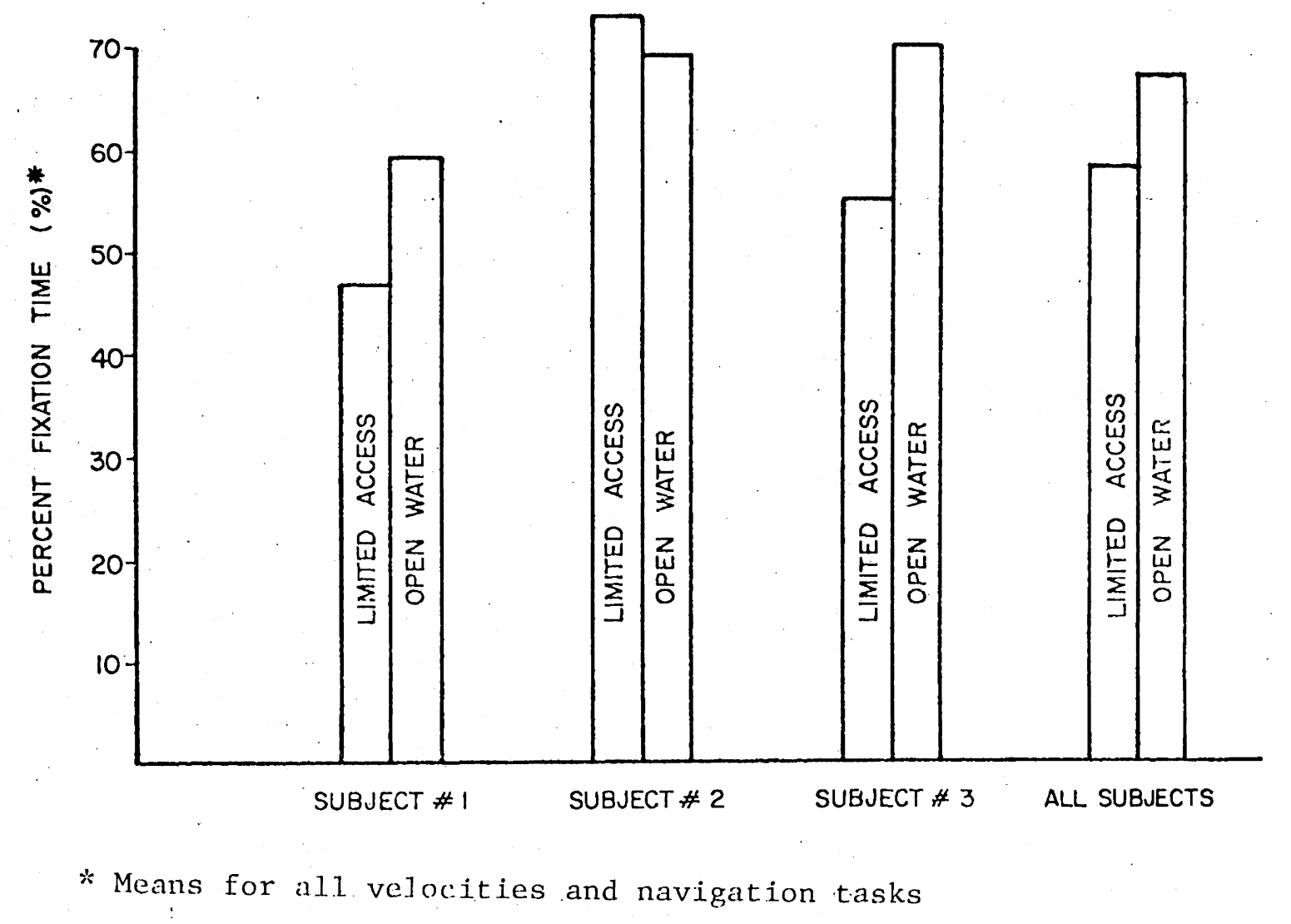

Figure D.8: Subject-boating environment effects on percent fixation time in the center visual zone 


\section{Subject Velocity Interaction Effect}

The final subject interaction had to do with the effect of velocity (see Figure D.9). Subject \#2 spent approximately the same portion of time fixating in this central zone regardless of the velocity. Subjects $\|_{1}$ and 3 , normally with simflar test results, are opposite in this case. Figure D.9 indicates that Subject 1 I reduced his percent fixation time in this center zone as speed increases, while Subject 33 increased the percent fixation time as speed increased. Subject 非 had equivalent percent fixation percentages as Subject 非 at the medium and high velocities, while Subject $\#_{1}$ was always significantly lower than Subject $\left.\right|_{2}$ at all veloclties.

\section{Components of Variance for the Visual Zones}

In an attempt to further illustrate some of the previous effects, the components of variance were determined and are 111 ustrated in Figure D.10. As expected, the unexplained portion is large for the left visual zone since the subject independent variable was the only significant parameter (see Table D.3). The error term is smaller for the right vs. left visual zone since the task effect accounts for $40 \%$ of the variance, However, for the center visual zone, the task effect is reduced (18\%) with the subject and subject-task effect accounting for over $50 \%$ of the varlance. Again, many of these effects can be accounted for by the different behavior patterns of Subject \#2. His reduced scanning patterns resulted in larger percent fixation times in this center zone. 


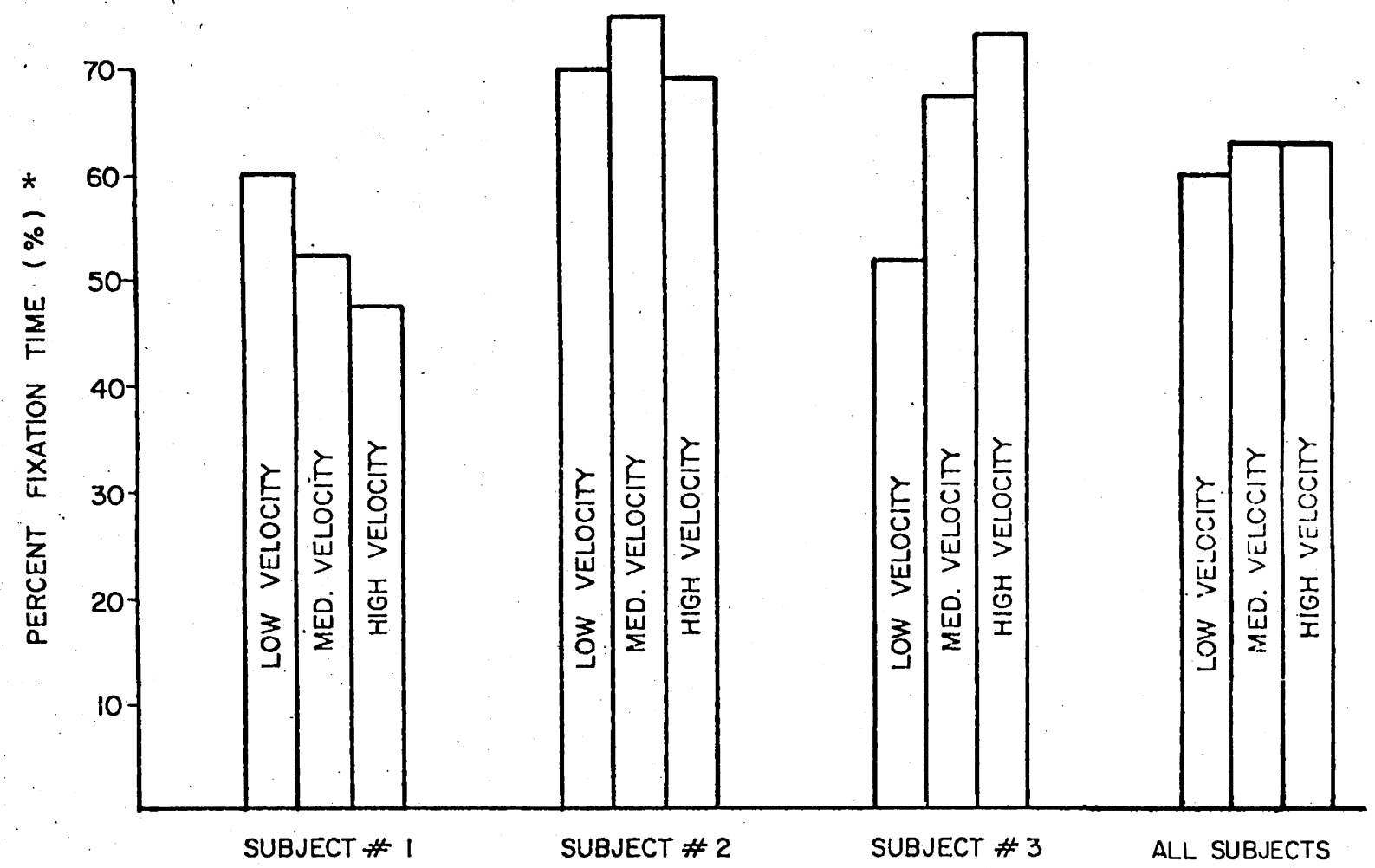

*Means for both boating environments and all navigation tasks

Figure D.9: Subject-velocity effects on percent fixation time in the center visual zone 


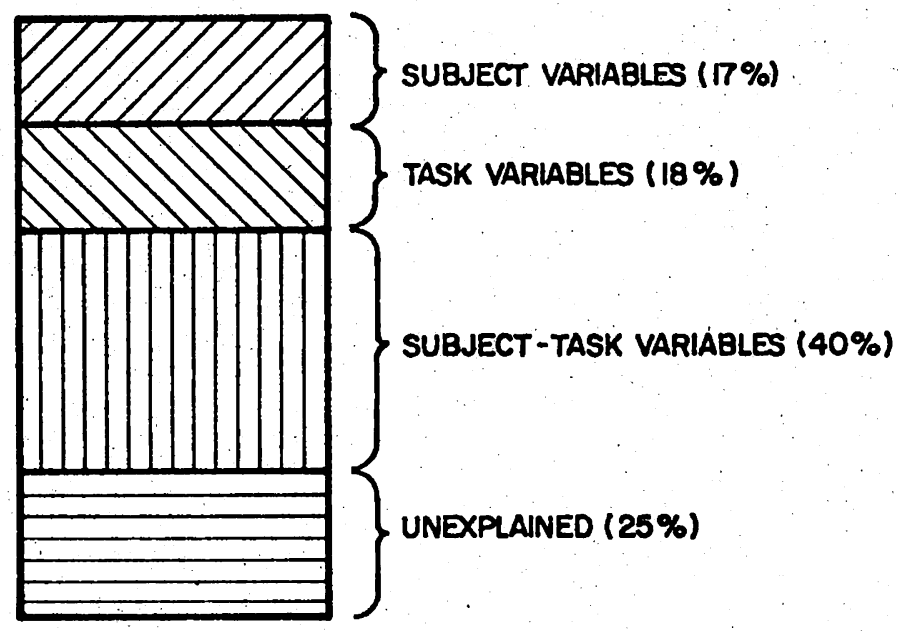

CENTRAL VISUAL ZONE

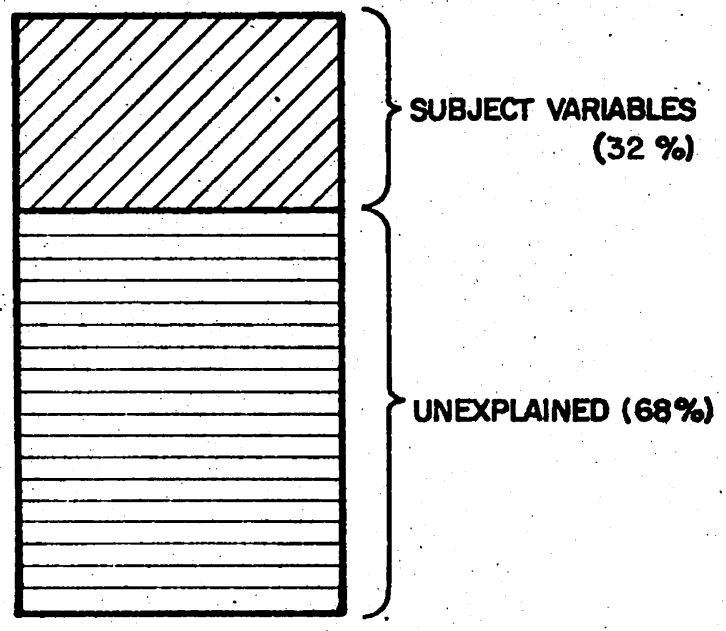

LEFT VISUAL ZONE

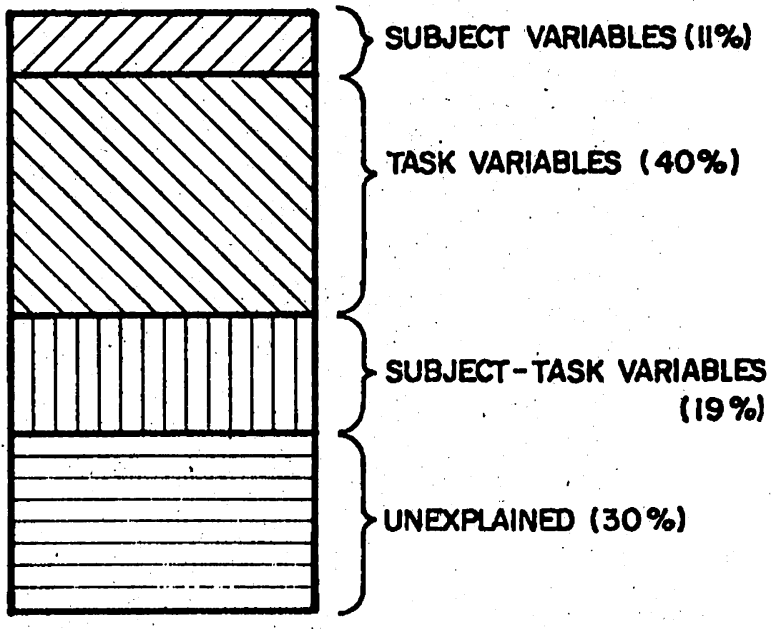

RIGHT VISUAL ZONE

Figure D.10: Components of variance for the visual zones 


\section{APPENDIX E}

\section{PREDICTION OF BOATERS' SCANNING BEHAVIOR}

Title

Prediction of Boaters" Scanning Behavior

Prediction of Horizontal Fixation Locations. 158

Prediction of Vertical Fixation Locations

162

Table

Page.

Table E.1: Polynomial Orthogonal Contrasts Used in Regression Equations

Table E.2: Summary of Prediction Equations for Horizontal

Table E.3: Prediction of Mean Horizontal Location from Regression Equation

Table E.4: Prediction of Standard Deviation of Horizontal Location from Regression Equation

Table E.5: Prediction of Mean Vertical Location from Regression Equation

Table E.6: Prediction of Standard Deviation of Vertical

Location from Regression Equation 


\section{PREDICTION OF BOATERS' SCANNING BEHAVIOR}

The analysis of results can be further extended by utilizing stepwise multiple regression models to determine the relative importance of the variables already found to be significant in the previous ANOVA tables. Because many of the independent variables were at three levels, it is also possible to determine the linear and quadratic effects of these variables on the dependent variables. In order to accomplish this, a model of the following form was utilized :

$$
\begin{aligned}
Y_{i j k 1 m} & =A_{0}+A_{1} V_{i(1 i n)}+A_{2} V_{i \text { (quad) }}+A_{3} S_{j(1 i n)}+A_{4} S_{j \text { (quad) }} \\
& +A_{5} E_{k}+A_{6} T_{\ell(1 i n)}+A_{7} T_{\ell \text { (quad) }}+A_{8} V_{i(1 i n)} S_{j(1 i n)} \\
& +A_{9} V_{i(1 i n)} S_{j(\text { quad) }}+A_{10} V_{i \text { (quad) }} S_{j(1 i n)}+A_{11} V_{i \text { (quad) }} S_{j \text { (quad) }} \\
& +. .+ \text { error. }
\end{aligned}
$$

where: $v_{i}=$ Velocity, $i=1-3$

$$
\begin{aligned}
\mathrm{S}_{\mathrm{j}} & =\text { Subject, } \mathrm{j}=1-3 \\
\mathrm{E}_{\mathrm{k}} & =\text { Boating Environment, } \mathrm{k}=1 ; 2 \\
\mathrm{~T}_{\ell} & =\text { Navigation Task, } l=1-3 \\
(\text { (in) } & =\text { Linear Contrasts } \\
(\text { quad }) & =\text { Quadratic Contrasts }
\end{aligned}
$$

To determine the linear or quadratic effects listed in the previous equation, polynomial orthogonal contrasts as defined by Hicks (1973) were utilized. The particular contrasts for each main 
effect are listed in Table E.1. These particular contrasts compare two levels of an independent variable against a third leve1. Thus, Subject 非 was compared against Subjects $\|_{1} 1$ and $\|_{3}$, and the centering in channel task was compared against the compass and visual reference point tasks. This is indicated by the different weightings as listed in Table E.1 (e.g., to determine the quadratic effect related to Subject 非, the weighting factor is 2). These decisions were made post hoc based on the differences discussed in Chapter VI However, it was not evident from the previous analysis which velocity level to use to compare to the remaining two levels. Therefore, all combinations were run and it was determined that testing the low and high velocity versus the medium velocity resulted in the best prediction equations. A summary of all significant variables in the regression equations is contained in Table E.2. Many of the resultant regression equations are extremely lengthy due to determining both the iinear and quadratic effects of the independent variables (e.g., Subject ${ }_{\ell}$ or Task $_{q}$ ). The results differ at times from the ANOVA's summarized in Table 4.5 because the ANOVA's do not partition out the linear and quadratic effects. Due to the complexity of the equations, the following discussion is separated into the prediction of the two measures, horizontal and vertical eye fixations. 
Table E.1

Polynomial Orthogonal Contrasts Used In Regression Equations

\begin{tabular}{|c|c|c|}
\hline & \multicolumn{2}{|c|}{ Orthogonal Contrasts } \\
\hline Independent Variable & $\begin{array}{c}\text { Linear } \\
\text { Coefficients }\end{array}$ & $\begin{array}{l}\text { Quadratic } \\
\text { Coefficients }\end{array}$ \\
\hline Veloctty: & s & \\
\hline Low Velocity & 1 & 1 \\
\hline Med Velocity & 0 & -2 \\
\hline High Velocity & -1 & 1 \\
\hline Subject: & & \\
\hline $\mathrm{S}$ 非1 & 1 & 1 \\
\hline$S$ 非 2 & 0 & -2 \\
\hline $\mathrm{S} \cdot \mathbb{B}_{3}$ & -1 & 1 \\
\hline Boating Environment: & & \\
\hline Limited Access & 1 & \\
\hline Open & -1 & \\
\hline Tasks : & & . \\
\hline Compass & 1 & 1 \\
\hline Center in Channel & 0 & -2 \\
\hline Visual Ref. Pt. & -1 & 1 \\
\hline
\end{tabular}


Table E.2

Summary of Prediction Equations for Hortzontal and Vertical Fixation Measures ${ }^{1}$

\begin{tabular}{|c|c|c|c|c|}
\hline & \multicolumn{2}{|c|}{ Horizontal $\left({ }^{\circ}\right)$} & \multicolumn{2}{|c|}{ Vertical $\left({ }^{\circ}\right)$} \\
\hline & Mean & $\begin{array}{l}\text { Standard } \\
\text { Deviation }\end{array}$ & Mean & $\begin{array}{l}\text { Standard } \\
\text { Deviation }\end{array}$ \\
\hline Constant & -3.6 & -40 & -2.1 & 4.6 \\
\hline \multirow{2}{*}{\multicolumn{5}{|c|}{$\begin{array}{l}\text { Independent } \\
\text { Variables: } \\
\mathrm{v}_{\ell}\end{array}$}} \\
\hline & & & & \\
\hline \multicolumn{5}{|c|}{$\mathrm{V}_{\mathrm{g}}$} \\
\hline \multicolumn{5}{|l|}{$\mathrm{s}_{\ell}$} \\
\hline $\mathrm{S}_{\mathrm{q}}$ & -1.1 & 2.3 & & \\
\hline $\mathrm{E}$ & 2.0 & 3.1 & & \\
\hline $\mathrm{T}_{\ell}$ & -1.4 & -3.6 & & \\
\hline $\mathrm{T}_{\mathrm{q}}$ & -1.7 & .6 & & \\
\hline $\mathrm{v}_{\ell} \mathrm{S} \ell$ & & -1.5 & & -.5 \\
\hline $\mathrm{v}_{\ell^{\mathrm{s}} \mathrm{q}}$ & & & & .3 \\
\hline $\mathrm{V}_{\mathrm{q}} \mathrm{S}$ & 1.1 & & & \\
\hline $\mathrm{V}_{\mathrm{q}} \mathrm{S}$ & & -.5 & & \\
\hline$V_{\ell^{E}}$ & & -1.4 & & \\
\hline \multicolumn{5}{|l|}{$\mathrm{V}_{\mathrm{q}} \mathrm{E}$} \\
\hline $\mathrm{v}_{\ell} \mathrm{T}_{\ell}$ & & & & .6 \\
\hline \multicolumn{5}{|l|}{$\mathrm{V}_{\ell^{\mathrm{T}}}$} \\
\hline $\mathrm{V}_{\mathrm{q}} \mathrm{T}_{\ell}$ & & 1.0 & & \\
\hline \multicolumn{5}{|l|}{$\mathrm{V}_{\mathrm{q}} \mathrm{T}_{\mathrm{q}}$} \\
\hline $\mathrm{S}_{\ell} \mathrm{E}$ & 1.6 & .9 & & \\
\hline $\mathrm{S}_{\mathrm{q}}^{\mathrm{E}}$ & 1.2 & .9 & & \\
\hline \multicolumn{5}{|l|}{$\mathrm{s}_{\ell} \mathrm{T}_{\ell}$} \\
\hline $\mathrm{S}^{\mathrm{T}} \mathrm{q}$ & & & & .2 \\
\hline
\end{tabular}


- Table E.2 (continued)

\begin{tabular}{|c|c|c|c|c|}
\hline \multirow[b]{2}{*}{$\cdots$} & \multicolumn{2}{|c|}{ Horizontal( $\left.{ }^{\circ}\right)$} & \multicolumn{2}{|c|}{ Vertical $\left(^{\circ}\right)$} \\
\hline & Mean & $\begin{array}{l}\text { Standard } \\
\text { Deviation }\end{array}$ & Mean & $\begin{array}{l}\text { Standard } \\
\text { Deviation }\end{array}$ \\
\hline $\mathrm{S}_{\mathrm{q}} \mathrm{l}$ & & & & .2 \\
\hline $\mathrm{S}_{\mathrm{q}} \mathrm{I} \mathrm{q}$ & $\checkmark$ & .4 & & \\
\hline EIe & -2.0 & -1.2 & & \\
\hline Fit & & & & \\
\hline $\mathrm{v}_{\ell} \mathrm{S}^{\mathrm{E}}$ & & & & \\
\hline $\mathrm{V}_{\ell} \mathrm{S} \mathrm{L}^{\mathrm{E}}$ & & & & \\
\hline $\mathrm{V}_{\mathrm{q}} \mathrm{S}^{\mathrm{I}}$ & & & & \\
\hline $\begin{array}{l}\mathrm{V}_{\mathrm{q}} \mathrm{S} \mathrm{I} \\
\end{array}$ & & & & .2 \\
\hline$e^{E I} \ell$ & & & & \\
\hline$e^{\mathrm{LT}} \mathrm{q}$ & -1.2 & & & \\
\hline $\mathrm{V}_{\mathrm{qT}} \mathrm{e}$ & & & & \\
\hline $\mathrm{V}_{\mathrm{q}} \mathrm{El} \mathrm{q}$ & -.6 & & & -.1 \\
\hline $\mathrm{V}_{\ell^{\mathrm{S}}} \ell^{\mathrm{I}} \ell$ & & & & $\because$ \\
\hline $\mathrm{V}_{\ell} \mathrm{S}^{\mathrm{T} \mathrm{T}^{\mathrm{T}} \mathrm{q}}$ & 1.9 & & $\ddots$ & .3 \\
\hline $\mathrm{V}_{\ell} \mathrm{S}_{\mathrm{q}}^{\mathrm{T}} \mathrm{q}$ & $\because$ & & & \\
\hline 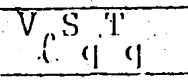 & & -.6 & & .2 \\
\hline $\mathrm{q}^{\mathrm{S}} \ell^{\mathrm{I}} \ell$ & & & & \\
\hline $\mathrm{q}^{\mathrm{S}} \ell^{\mathrm{I}} \mathrm{q}$ & & & & \\
\hline $\mathrm{q}^{\mathrm{S}} \mathrm{q}^{\mathrm{T}} \ell$ & & .7 & & \\
\hline V. $q^{2} q^{\prime \prime} q$ & & $\cdot-.4$ & & .2 \\
\hline$S_{i}\left[E^{\prime}{ }^{\prime}\right.$ & . & -1.5 & & \\
\hline $\mathrm{S}_{\ell} \mathrm{ET} q$ & & -1.2 & & .2 \\
\hline $\mathrm{S}_{\mathrm{q}} \mathrm{ET}_{\mathrm{C}}$ & -1.9 & & & \\
\hline $\mathrm{S}_{\mathrm{q}} \mathrm{FI}$ & $\because$ & & & \\
\hline $\mathrm{V}_{\ell} \mathrm{S}_{\ell} \mathrm{ET}_{\ell}$ & & & & \\
\hline $\mathrm{V}_{\ell} \mathrm{S}_{\ell} \mathrm{ET}_{4}$ & & & & .6 \\
\hline $\mathrm{V}_{\ell} \mathrm{S}^{\mathrm{ET}} \ell$ & -1.3 & & .4 & -.5 \\
\hline $\mathrm{V}_{\mathrm{C}_{\mathrm{q}} \mathrm{ET}}$ & & & & \\
\hline
\end{tabular}


Table E. 2 (continued)

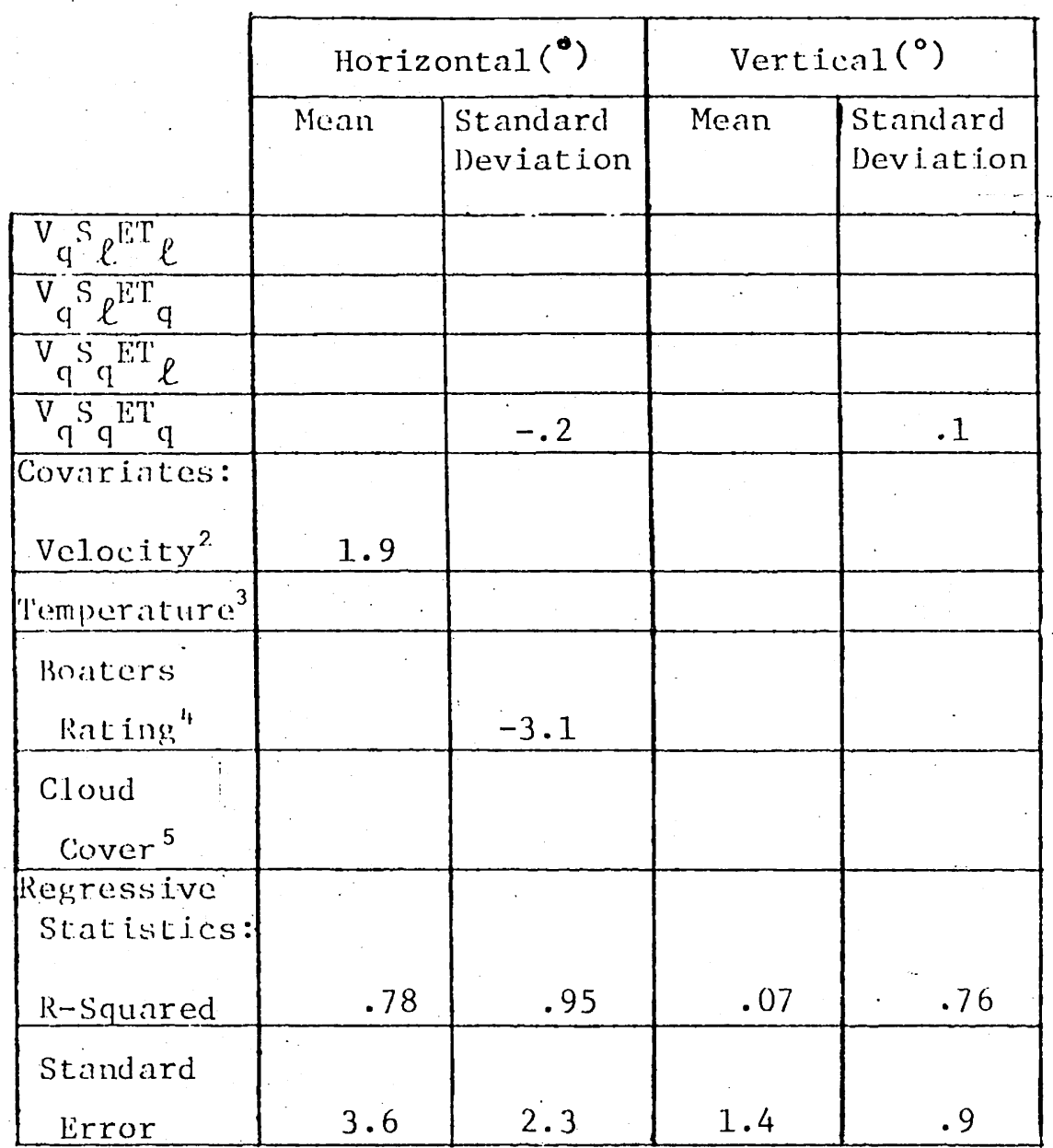

where: $\quad \mathrm{V}=$ Velocity

$\mathrm{S}=$ Subject

$\mathrm{T}=$ Navigation Task

$\dot{\mathrm{E}}=$ Boating Environment

$\ell=$ Linear Contrast

$\mathrm{q}=$ Quadratic Contrast

${ }^{1}$ Numbers in the cells indicate significant variables and their coefficients.

${ }^{2}$ Not an Orthogonal Contrast; Low Velocity $=1$, Medium $=2$, and High $=3$.

${ }^{3}$ Temperature $\left({ }^{\circ} \mathrm{F}\right)$ during testing.

${ }^{4}$ Subjects' boating skill ratings.

${ }^{5}$ Percent Cloud Cover during testing. 


\section{PREDICTION OF HORIZONTAL FIXATION LOCATIONS}

It is obvious from Table E. 2 that neither of the horizontal parameters have simple predictive equations nor will the discussion of these equations be effortless.: For the standard deviation of horizontal location, the amount of variance that the horizontal equations in Table E.2 accounts for is extremely good $\left(r^{2}=.92\right)$ and adequate for the mean horizontal location $\left(r^{2}=.78\right)$. The coefficients of the variables for mean horizontal location are surprisingly similar with most of them ranging from $1.1^{\circ}$ to $2^{\circ}$.

Using these equations it is possible to predict means and standard deviations of horizontal location and these predictions are listed in Tables E.3 and E.4. For example, Table E.3 indicates that in the limited access water condition during both the visual reference point and centering in channel tasks, the mean was almost always to the right of straight ahead; whereas, for the compass task, the mean was almost always left of straight ahead.

In the open water boating environment, the compass task mean horizontal location was, again, almost always left of straight ahead and the visual reference point task's mean horizontal location was also left of straight ahead for Subjects $\# 1$ and \#3. However, the open water centering in channel task was almost evenly divided between being to the right or left of straight ahead.

Although the data utilized to develop these regression equations did not contain fixations to the navigation target, they still produced a bias in the mean location. As an example, consider the 


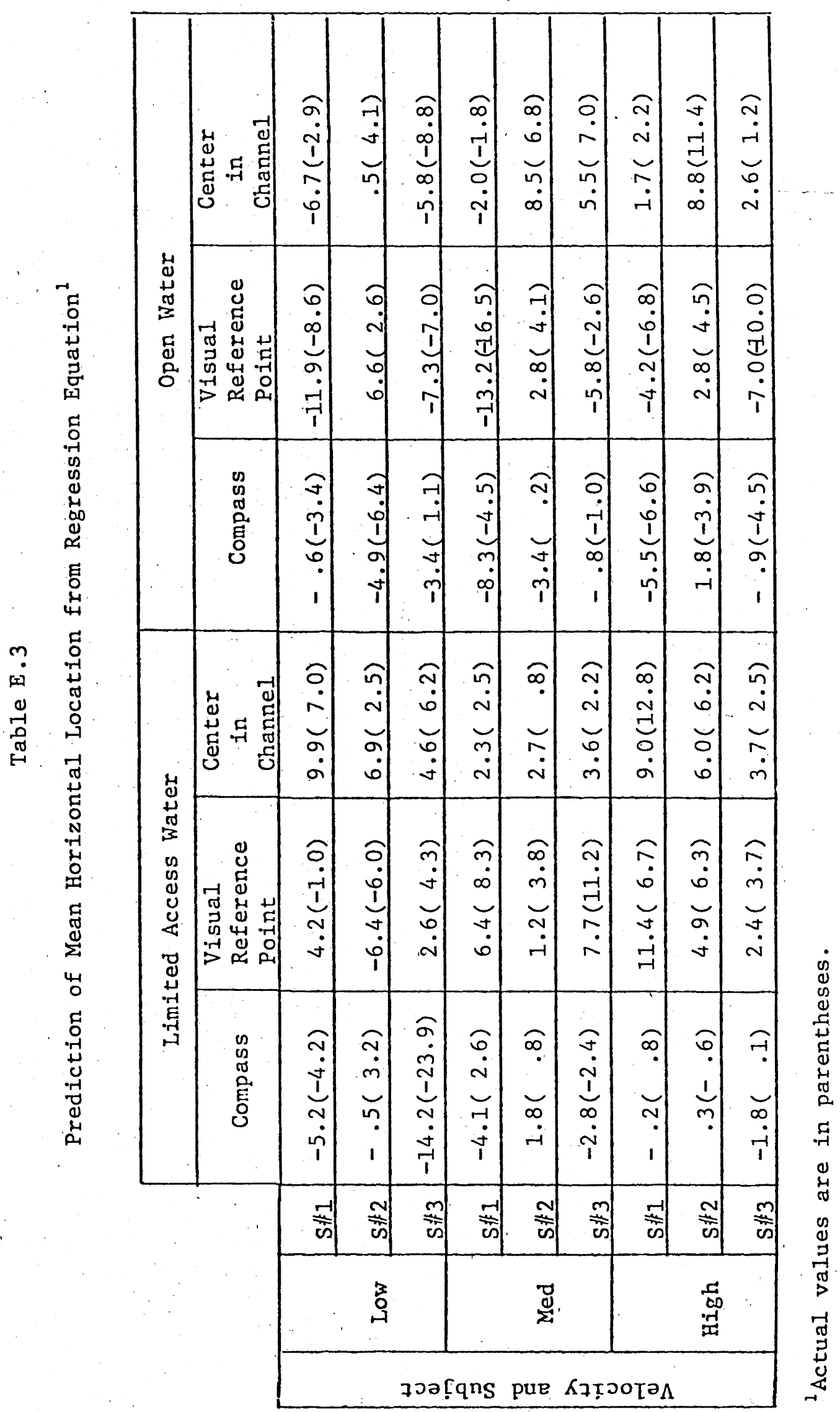




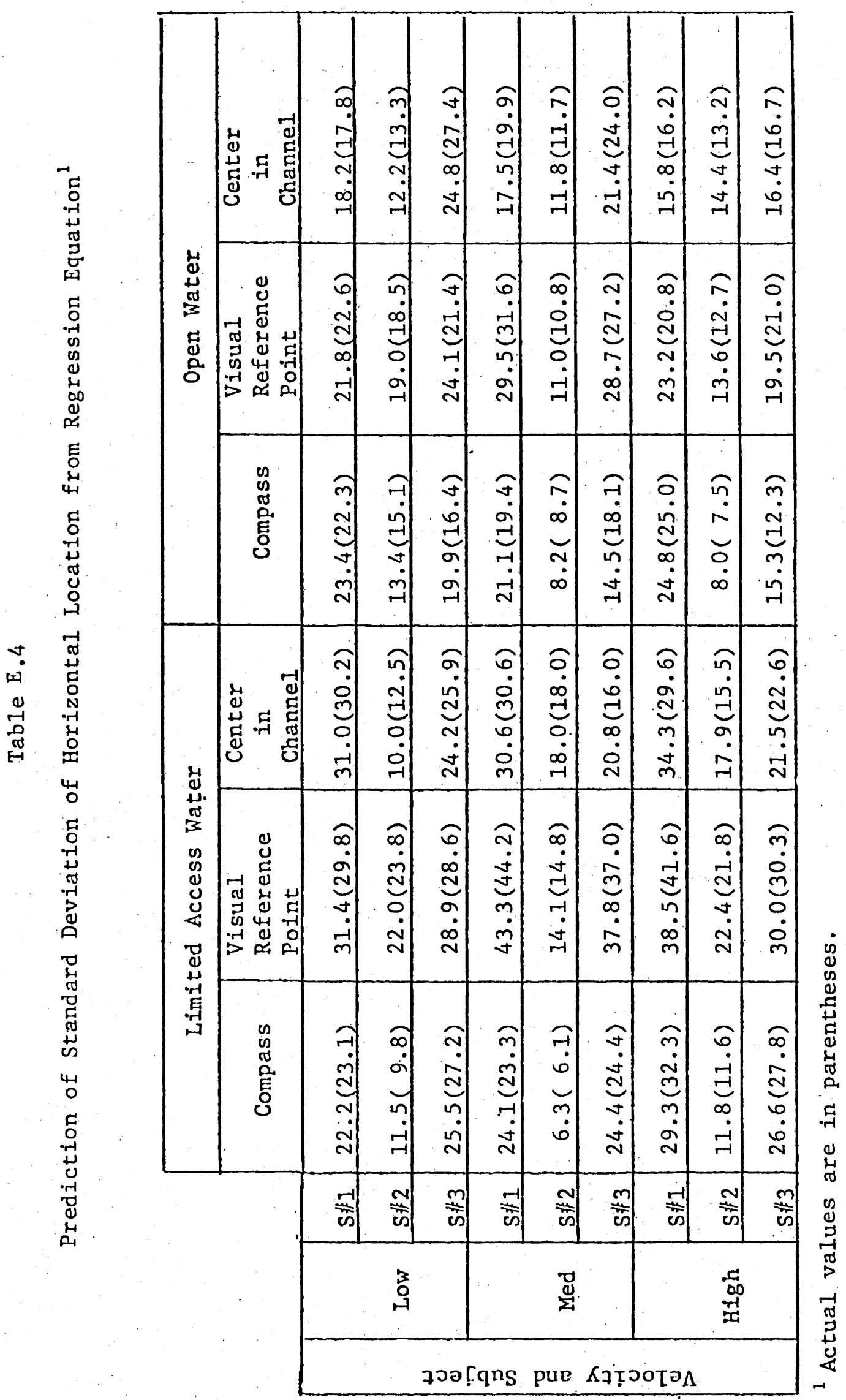


compass task. The compass is located at approximately $-40^{\circ}$ to the left of straight ahead, and boaters switching between looking at the compass and scanning the water straight ahead scanned to the left of straight ahead. This may be a conservation measure in order to reduce the amount of eye and head travel in between fixations. In the visual reference point task, the navigation target, either a smoke stack or water tower, was straight ahead of the boater. Thus, one would not expect that the data was biased due to the location of the navigation target for the visual reference point task unless another variable, uncontrolled in the study, affected these mean locations. While performing the centering in channel task, the boater was supposed to keep the vessel in the center of the channel as marked by buoys. A bias toward the right of straight ahead may indicate that the boater favored the buoys to the right of his vessel.

Analysis of Table E.4 indicates that Subject 非, as previous1y discovered, always had a smaller scanning pattern, as depicted by the horizontal standard deviation, than Subjects $\# 1$ and \#3. Furthermore, as previously discussed, the limited access water conditions almost always had a larger standard deviation than the open water condition. The smaller set of standard deviations, consistent for all subjects, was in the open water centering in channel, high speed situation. In fact, Subject 非's smallest deviations were always in the open water centering in the channel situations, while Subject 非's smallest scan patterns were displayed in the open water compass situation and were consistent through all velocities. The reasons for the 
difference between the boating environments has already been proposed in Chapter III. Due to the fact that the prediction equation for standard deviation of horizontal location accounted for so much variance $\left(r^{2}=.95\right)$ the predictions from these regressions further amplifies these findings.

\section{PREDICTION OF VERTICAL FIXATION LOCATIONS}

Although the ANOVA tables did not reveal any significant effects for the vertical components, the regression equations contained in Table E. 2 did produce variables that have a significant effect on the vertical parameters. The equation for mean vertical location does not account for much variance $\left(r^{2}=07\right)$; however, predictions were still developed and are contained in Table E.5, These predictions illustrate that during the centering in channel task, across both boating environments, all subjects and all velocities, there was a consistent mean vertical location. Furthermore, a11: tasks for a11 subjects in both boating environments at the second speed had the same mean vertical location parameter. In addition to these results, Subjects $\# 1$ and $\# 3$ had equivalent mean vertical locations for al1 three velocities. Additional discussion of results from these regression equations is not warranted due to the poor prediction qualities of this equation.

The regression equation for the standard deviation of vertical location as listed in Table E. 2 is a much better predictor $\left(r^{2}=.76\right)$; 


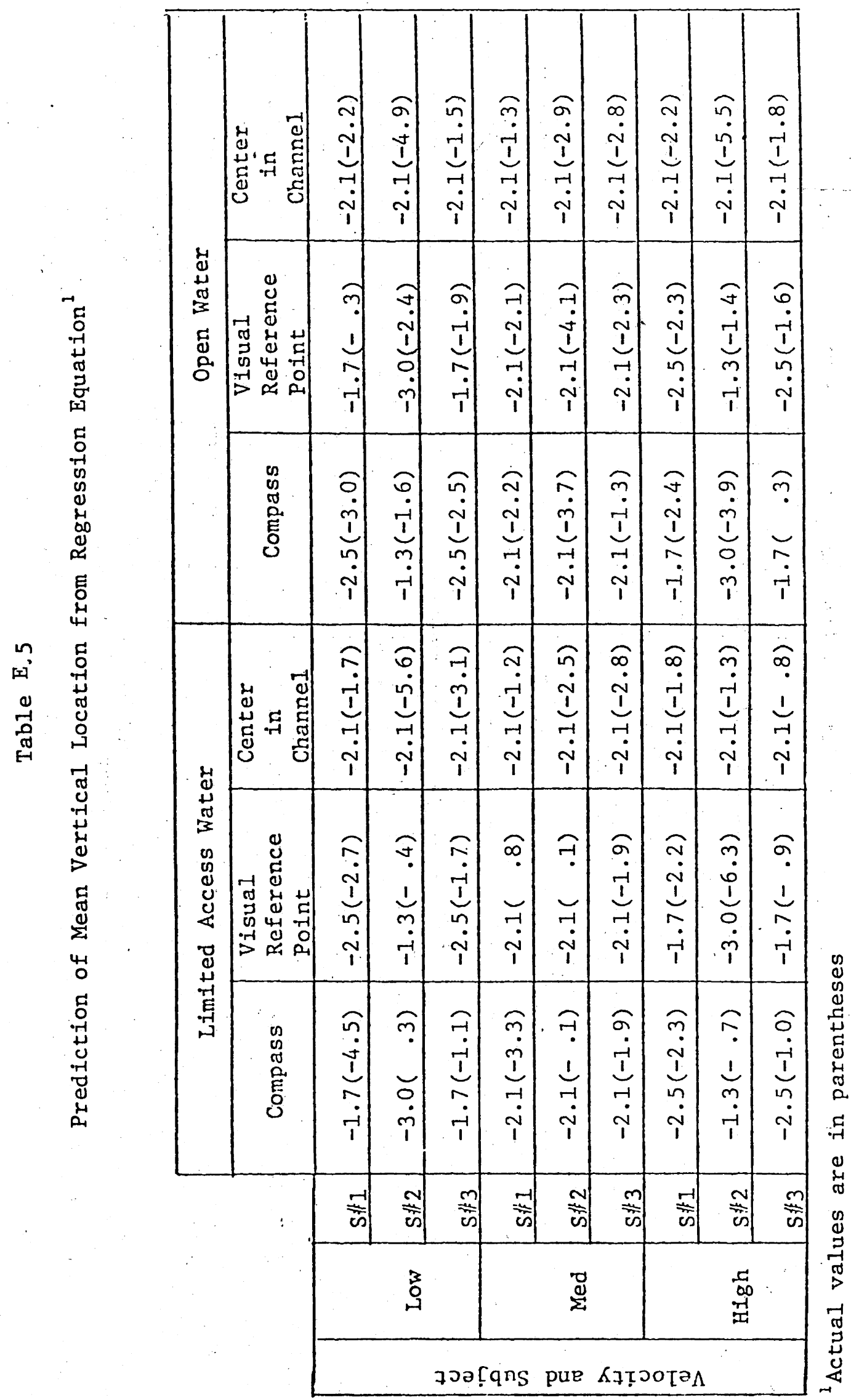




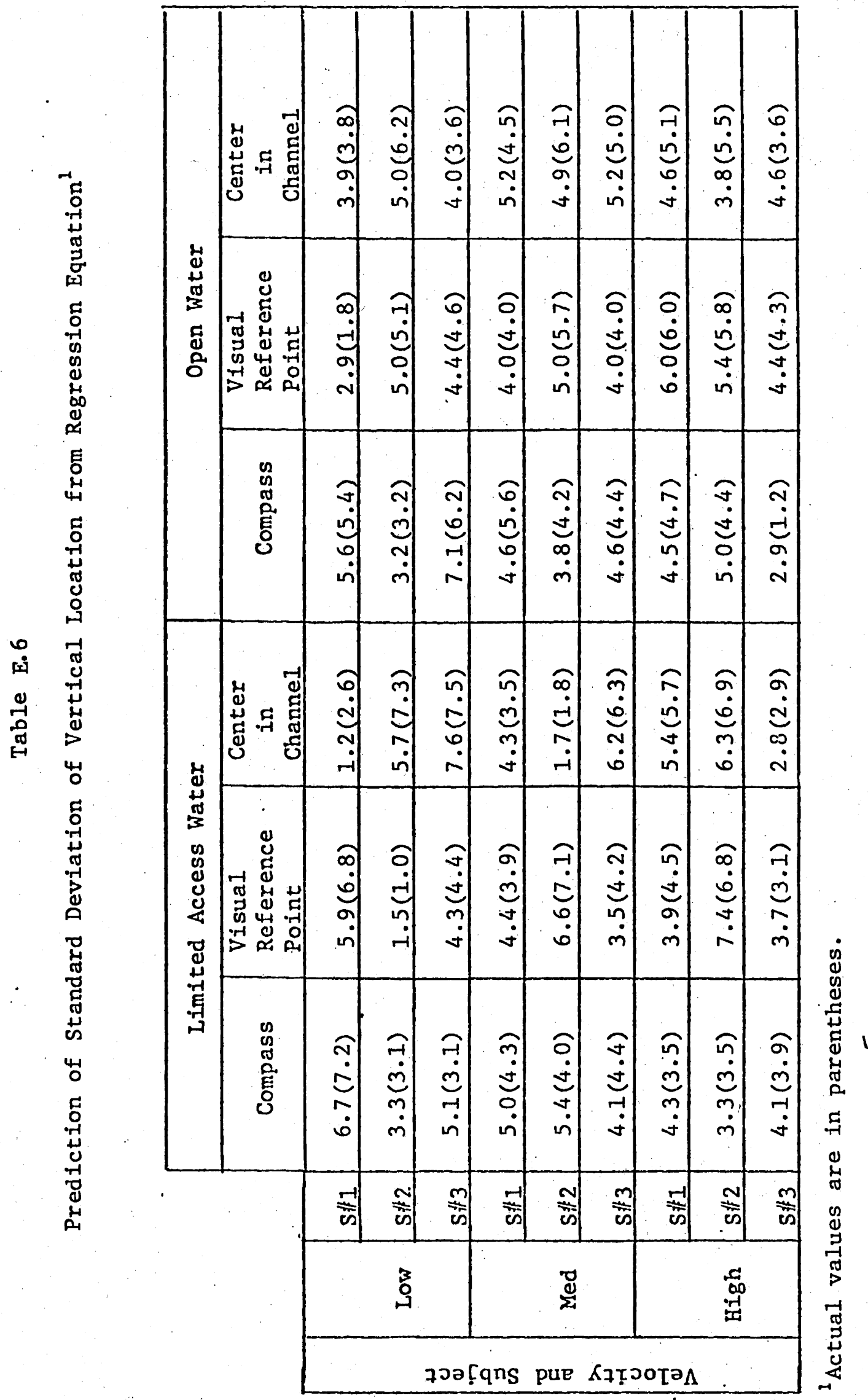


on the same order as the regression equation for mean horizontal location. The components for the variables listed in Table E.2 are a better weighting factor than have previously been found. By this, it is meant that some of the components are twice the value of the others (i.e., . 5 ot .6 versus .2 or .3 as noted in Table E.2). Again, predictions were developed from this regression equation and are contained in Table E.6. The open water task condition had more consistent measures of vertical standard deviation, with none of the numbers being smaller than $2.9^{\circ}$ and only one condition being greater than $6^{\circ}$. Whereas during the limited access water condition, four of the task situations had a small standard deviation below $3^{\circ}$, and six of the conditions are greater than $6^{\circ}$. Although this $3^{\circ}$ and $6^{\circ}$ cut-off criteria were arbitrarily selected, they are used as indications of small and large vertical scanning patterns.

In summary, the regressions developed resulted in lengthy equations which had high R-Squares (a11 except one was above .75). An overview of Table E.2 indicates that the main effects were significant in many of the equations. Since many quadratic effects were significant. future research should continue with three levels. 




\section{Highway Safety Resenroh Instiute}



JAN 291980

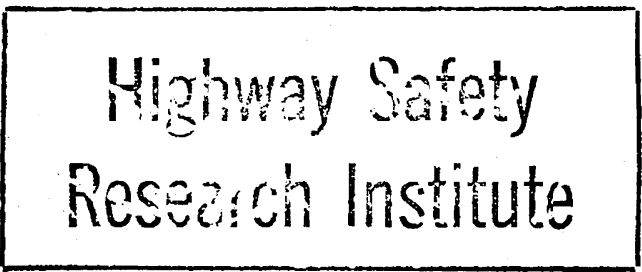

